\title{
Solid State Lighting Program
}

FINAL TECHNICAL REPORT

Date Prepared: February 12, 2008

Agreement Title: Low-Cost Blue/UV LEDs with Very High Photon Conversion and Extraction Efficiency for White Lighting

Work Performed Under Agreement:

DE-FC26-04NT42275

Submitted By: Boston University, 8 Saint Mary's Street

Boston MA 02215

Principal Investigator:

Professor Theodore D. Moustakas

Phone Number: 617-353-5431

Fax Number: 617-353-6440

E-Mail: Moustakas@bu.edu

Submitted To:

U. S. Department of Energy

National Energy Technology Laboratory

COR: Ryan Egidi

E-Mail: Ryan.Egidi@netl.doe.gov 


\section{DISCLAIMER}

This report was prepared as an account of work sponsored by an agency of the United States Government. Neither the United States Government nor any agency thereof, nor any of their employees, makes any warranty, express or implied, or assumes any legal liability or responsibility for the accuracy, completeness, or usefulness of any information, apparatus, product, or process disclosed, or represents that its use would not infringe privately owned rights. Reference herein to any specific commercial product, process, or service by trade name, trademark, manufacturer, or otherwise does not necessarily constitute or imply its endorsement, recommendation, or favoring by the United States Government or any agency thereof. The views and opinions of authors expressed herein do not necessarily state or reflect those of the United States Government or any agency thereof. 


\section{Table of Contents}

1. Task Update........................................................ 3

2. Task 1 -_Growth and Fabrication of LEDs............................ 3

Task 1.1: Development of GaN Templates Having Random Textured Surfaces by HVPE ...3

Task 1.2: Epitaxial Growth of $\mathrm{n}-$ and $\mathrm{p}-\mathrm{GaN}$ and $\mathrm{AlGaN}$ Layers by MBE and MOCVD on Textured GaN Templates....................................... 17

Task 1.3: Epitaxial growth of InGaN / AlGaN single and Multiple Quantum Wells by MBE and MOCVD on Textured GaN Templates......................... 19

Task 1.4: Epitaxial Growth of UV / Blue / Green LEDs by MBE and MOCVD on Textured GaN Templates.................................................. 37

Task 1.5: Fabrication and characterization of LED structures on smooth and textured $\mathrm{GaN}$ templates...........................................40

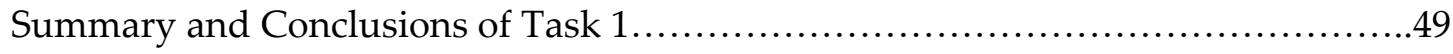

3. Task 2 - Theory and Simulations.......................................... 50

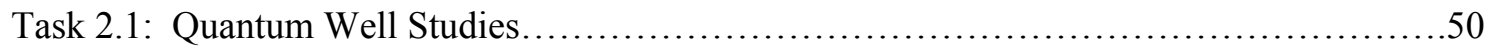

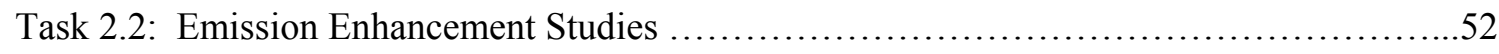

Task 2.3: Light Extraction Angle Studies.........................................54

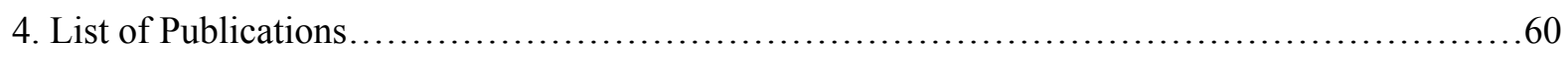

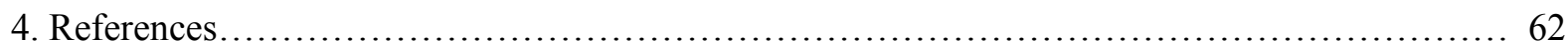




\section{Low-Cost Blue/UV LEDs with Very High Photon Conversion and Extraction Efficiency for White Lighting}

\section{0 Task Update}

The project had two main tasks: One addressed the materials and device development and it was carried out at Boston University. The second addressed the theory and simulation of materials and devices and it was carried out at Science Application International Corporation (SAIC). Each task had a number of sub-tasks which are described in the following table. Progress in these tasks is described in this section.

\section{Task 1 - Growth and Fabrication of LEDs (BU)}

Task 1.1 Development of GaN Templates Having Random Textured Surfaces by the HVPE Method

Task 1.2 Epitaxial Growth of $\mathrm{n}$ - and $\mathrm{p}-\mathrm{GaN}$ and AlGaN Layers by MBE and MOCVD on Textured GaN Templates

Task 1.3 Epitaxial Growth of InGaN / AlGaN Single and Multiple Quantum Wells by MBE and MOCVD on Textured GaN Templates

Task 1.4 Epitaxial Growth of UV / Blue / Green LEDs by MBE and MOCVD on Textured GaN Templates

Task 1.5 Fabrication and Characterization of LED Structures on Textured GaN Templates

Task 2 - Theory and Simulations (SAIC)

Task 2.1 Quantum Well Studies

Task 2.2 Emission Enhancement Studies

Task 2.3 Light Extraction Angle Studies

Task 1 - Growth and Fabrication of LEDs

Task 1.1: Development of GaN Templates Having Random Textured Surfaces by HVPE:

Summary: In this sub-task we addressed the following issues:

- Optimization of the vertical HVPE reactor for the growth of GaN templates

- Thermal treatment of Sapphire substrate to generate atomic steps

- Deposition of atomically smooth and randomly textured 50 to $100 \mu \mathrm{m}$ thick (0001) GaN template on (0001) sapphire substrates.

- Deposition of thick non-polar (11-20) GaN templates on (10-12) sapphire substrates.

- Characterization of the templates and substrates. 


\section{A. Deposition System and methods of growth}

The growth of the GaN templates took place in a home made HVPE reactor having a vertical geometry as schematically shown in Figure 1 . The system consists of a three zone furnace and the various gases were delivered using mass flow controllers (MFC).

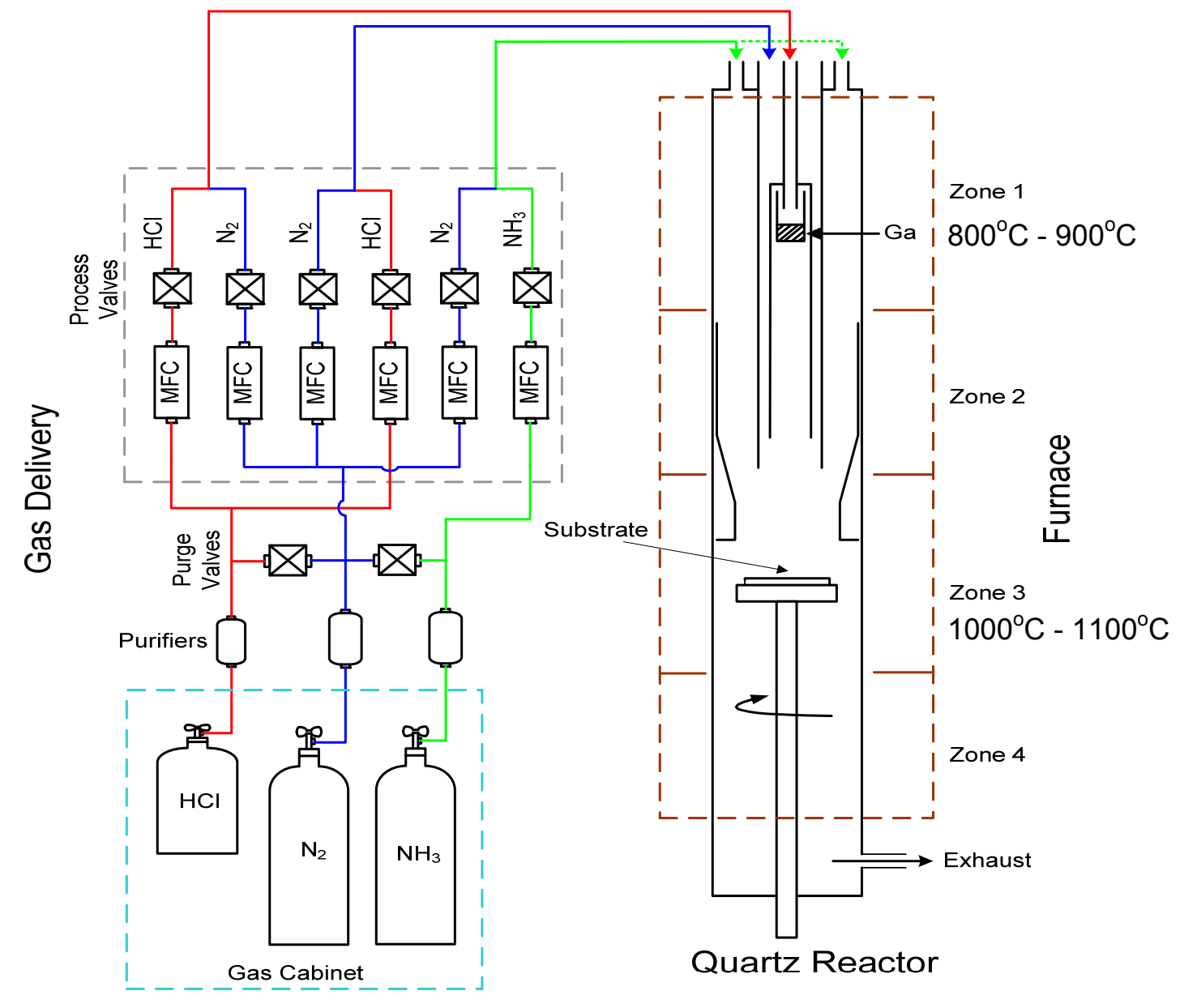

Figure 1: Schematic of the HVPE system used for the growth of GaN templates

There are three important reactions that takes place during the deposition of GaN by HVPE, two of which are the formation of the $\mathrm{Ga}$ and $\mathrm{N}$ precursors, and the third one is the formation of $\mathrm{GaN}$. The first step is the formation of $\mathrm{GaCl}_{(\mathrm{g})}$, by the reaction of $\mathrm{Ga}_{(\mathrm{l})}$ and $\mathrm{HCl}_{(\mathrm{g})}$. The extent of this reaction depends on three factors, namely, the temperature at which the reaction takes place, the residence time of $\mathrm{HCl}_{(\mathrm{g})}$, and the surface area of the $\mathrm{Ga}_{(1)}$ which is exposed to $\mathrm{HCl}_{(\mathrm{l})}$. Studies have shown that the Ga conversion efficiency is almost $100 \%$ at temperatures between $850{ }^{\circ} \mathrm{C}$ to $950{ }^{\circ} \mathrm{C}[1]$.

The formation of the N-precursor is given by the thermal dissociation of $\mathrm{NH}_{3}$ on the surface of the substrate. The degree of dissociation of $\mathrm{NH}_{3}$ depends on its partial pressure, residence time in the reaction zone, and the catalytic nature of the environment in the reaction zone. However, at the growth temperature, the dissociation of $\mathrm{NH}_{3}$ is very low without the presence of a catalyst, 
and thus the conversion efficiency of $\mathrm{NH}_{3}$ is low. It has been reported that the dissociation of $\mathrm{NH}_{3}$ is only $<4 \%$ at the growth temperatures and therefore the $\mathrm{N}$-bearing species present throughout the reaction zone is mainly metastable, non-dissociated $\mathrm{NH}_{3}[2,1,3]$. As such, the partial pressure of $\mathrm{NH}_{3}$ barely changes and therefore doesn't influence much the growth rate of $\mathrm{GaN}$ [4]. GaN deposition is thermodynamically favorable at growth temperature $(\Delta \mathrm{G}=-2.2$ $\mathrm{kcal} / \mathrm{mole}$ at $1050{ }^{\circ} \mathrm{C}$ ). GaN is unstable and decomposes to its component elements at temperatures $>800{ }^{\circ} \mathrm{C}$. However, studies have shown that even though GaN decomposes at these elevated temperatures, the rate of decomposition is very slow [5]

During HVPE growth of GaN, substrate surface pretreatment methods, and buffer layers have been developed in order to enhance nucleation of $\mathrm{GaN}$ on such foreign substrates and reduce the defect densities of the epitaxial layer. Sapphire substrate surface preparations such as $\mathrm{GaCl}$ pretreatment [6] and nitridation with $\mathrm{NH}_{3}[7,8,9]$ have been shown to increase the heteronucleation density, reduce pit density, and improve the crystalline quality of the epitaxial GaN film. The use of buffer layers such as $\mathrm{ZnO}$ [10] or low temperature $\mathrm{GaN}[11,7,8,9]$ have also been shown to produce high quality GaN by HVPE. Studies have shown that the quality of the high temperature GaN layer is sensitive to the conditions of growth and subsequent annealing (to the growth temperature of the high temperature $\mathrm{GaN}$ film) of the buffer layer. Gu, et. al., have shown that a high growth rate to attain rapid initial nucleation and coalescence in growing the buffer layer gave an improvement in the background carrier concentration of the high temperature GaN bulk film . Tavernier, et.al., have shown that the annealing rate of the buffer to the growth temperature of the bulk film is critical. Slow annealing, done by keeping the thin buffer layer in the growth zone while ramping to $1050{ }^{\circ} \mathrm{C}$, produced secondary $\mathrm{GaN}$ orientations, (10 $\overline{1} 1) \mathrm{GaN} \|(0001) \mathrm{Al}_{2} \mathrm{O}_{3}$, as confirmed by XRD. Higher annealing rates of $100{ }^{\circ} \mathrm{C} / \mathrm{sec}$ eliminated these secondary orientations and produced high quality bulk GaN films. This high ramp rate was achieved by having a backflow region and a movable substrate holder in the HVPE system such that during growth, the buffer is kept in the backflow region at a low temperature while ramping up the growth zone to the growth temperature. Upon reaching the desired growth temperature, the sample is then moved to the growth zone thereby achieving a very high anneal rate.

The GaN smooth and textured templates were grown on $c$-plane sapphire substrates by the hydride vapor phase epitaxy (HVPE) method, and their morphology and optical properties were reported elsewhere. In this report we present only a brief discussion of the deposition conditions of the GaN templates. The GaN templates were produced in a custom-built vertical HVPE reactor employing independent temperature zones for heating the $\mathrm{Ga}$ boat and the substrate. GaN templates were grown in three steps. First, the (0001) sapphire substrate was heated to $1000{ }^{\circ} \mathrm{C}$ and was exposed to a flux of $\mathrm{GaCl}$ produced by passing $\mathrm{HCl}$ over $\mathrm{Ga}$, heated to $900{ }^{\circ} \mathrm{C}$ [11]. We believe that the exposure of the sapphire substrate to $\mathrm{GaCl}$ results in the removal of physisorbed species like oxygen and potentially carbon, which are harmful to epitaxial growth. Following this step, the substrate was cooled to $590{ }^{\circ} \mathrm{C}$ and a $\mathrm{GaN}$ buffer $(\sim 30$ $\mathrm{nm}$ thick) was deposited. The rest of the GaN template was grown at temperatures between 1070 ${ }^{\circ} \mathrm{C}-1100{ }^{\circ} \mathrm{C}$ under constant $\mathrm{NH}_{3}$-rich conditions. The growth rate was controlled by varying the flow rate of $\mathrm{HCl}$ over the $\mathrm{Ga}$ melt. Smooth $\mathrm{GaN}$ templates were grown at relatively low growth rates $(\sim 20 \mu \mathrm{m} / \mathrm{hr})$ and relatively high temperatures $\left(1100{ }^{\circ} \mathrm{C}\right)$. Both of these conditions promote lateral growth. The textured $\mathrm{GaN}$ templates were produced at higher flow rates of $\mathrm{HCl}$ over the Ga melt and lower temperatures $\left(1070^{\circ} \mathrm{C}\right)$. These conditions promote higher vertical growth and 
lead to rough surface morphology. Similar findings in producing GaN by the HVPE method were also reported by Tavernier [9]

\section{B. Preparation of the (0001) sapphire substrates}

Figure 2(a) shows the AFM surface morphology of a (0001) sapphire substrate as acquired from the manufacturer. The sapphire wafers, as delivered by the manufacturer, are decorated with a network of scratches due to mechanical polishing. These scratches are undesirable features during the nucleation of $\mathrm{GaN}$. We systematically annealed the substrates to $1400^{\circ} \mathrm{C}$ for approximately $1 \mathrm{hr}$. This process was found to lead to very smooth surface with well-defined step structure. The AFM surface morphology of such a wafer is shown in Figure 2 (b).

The step formation shown in Figure 2(b) is due to the miss-cut of the sapphire wafer. The manufacture specified surface orientation with an angular tolerance $+/-0.3^{\circ}$. The mechanism of step formation due to the misscut of the substrate is illustrated in Figure 3. At high temperature, atoms on sapphire surface become thermodynamically unstable. Specifically, mass migration takes place in order to reduce surface energy, tending to relax into positions corresponding to the equilibrium crystal shape. Terrace length is given by the expression:

$$
\mathrm{L}=\mathrm{c} / 6(\tan \theta)
$$

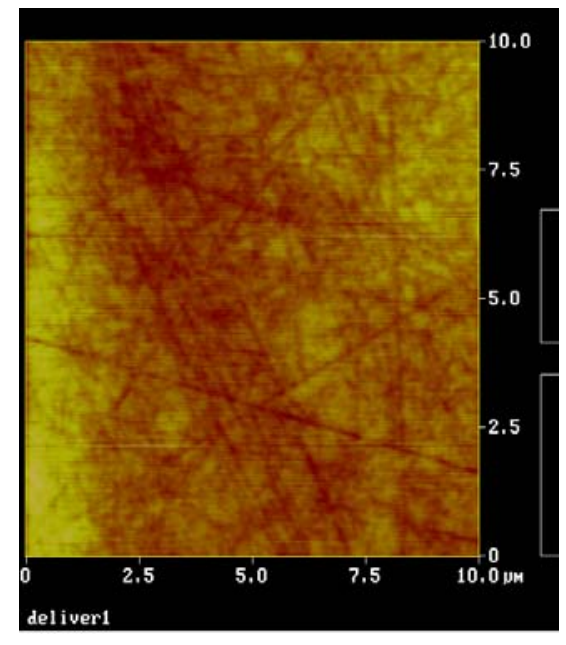

(a)

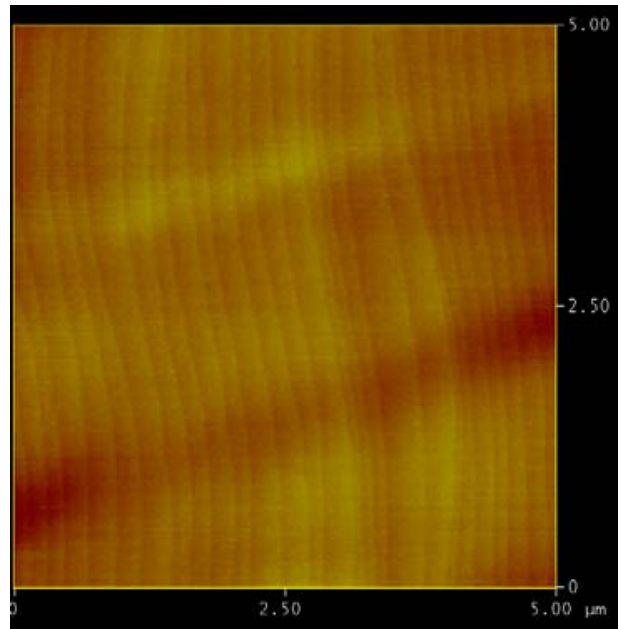

(b)

Figure 2: (a) AFM image of (0001) sapphire substrate as received from the manufacturer; (b) AFM of the same substrate after thermal annealing at $1400{ }^{\circ} \mathrm{C}$ for $1 \mathrm{hr}$. 


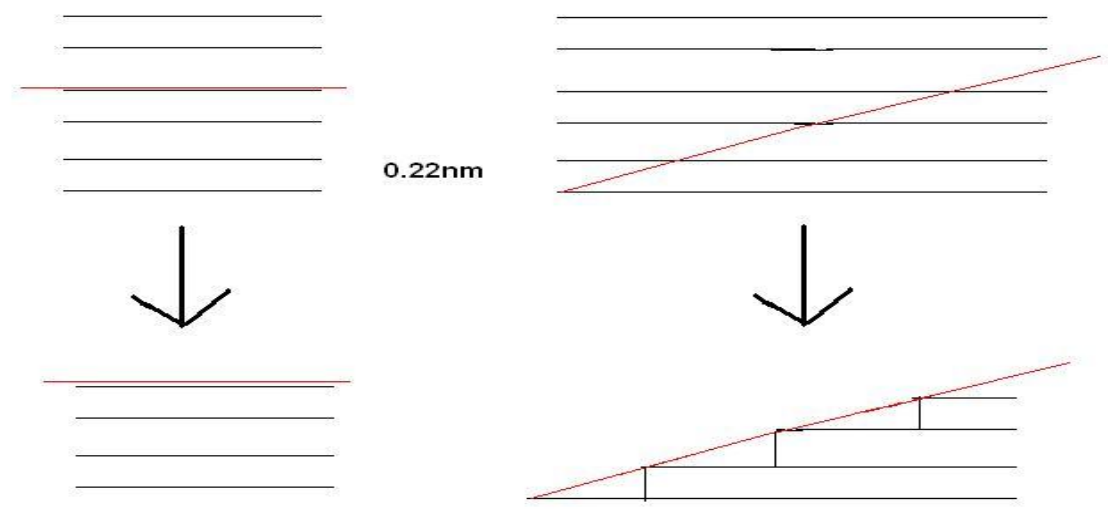

Figure 3: (Left panel) Sapphire unit cell cut on axis; (Right panel) Sapphire unit cell cut off axis

\section{Surface morphology of (0001) GaN templates grown by HVPE}

Films grown using the procedures described earlier have very smooth surface morphology. Figure 4 shows the cross-section and the surface SEM image of a $60 \mu \mathrm{m}$ thick GaN.
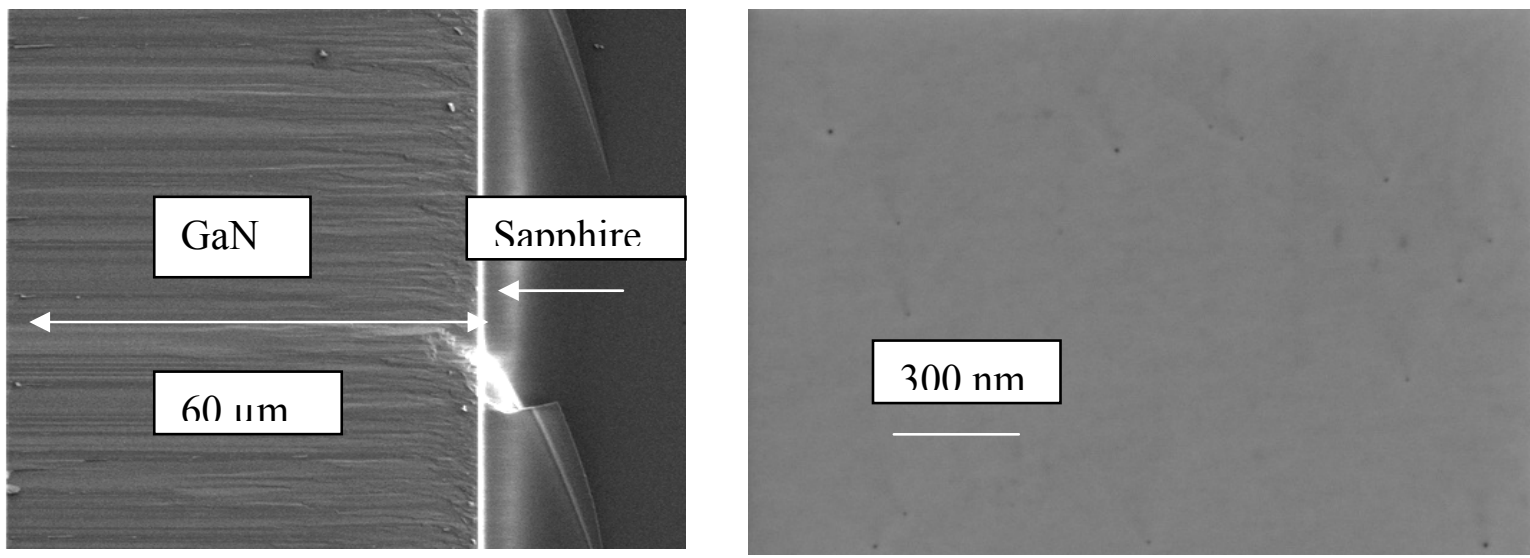

Figure 4: Cross-section (left panel) and surface (right panel) SEM image of a thick and smooth GaN film grown by the HVPE method.

As discussed previously other conditions lead to films with textured surface morphology. Figures 5 and 6 show SEM and AFM image of a smooth and a textured (0001) GaN template grown by HVPE. The smooth GaN template has an RMS roughness of $0.5 \mathrm{~nm}$ (atomically smooth) and the data of Figure 6 indicate that the 
growth mode was that of step flow. Depth analysis of several (0001) GaN textured templates shows Gaussian distribution (random distribution of surface texture) with average depths ranging from $0.8 \mathrm{~mm}$ to $3 \mathrm{~mm}$ depending of the deposition conditions. An example of such a depth analysis is shown in Figure 7.

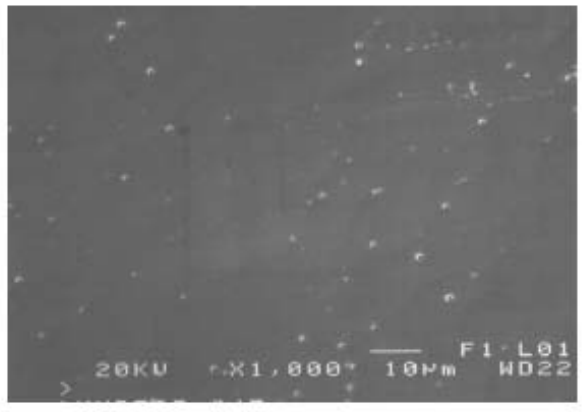

GaN smooth template

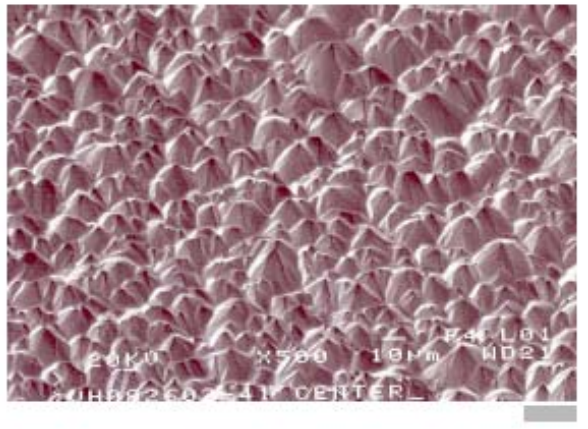

Spontaneously formed GaN textured template

Figure 5: SEM Surface Morphology of Smooth and Textured (0001) GaN Template deposited on (0001) Sapphire Substrates
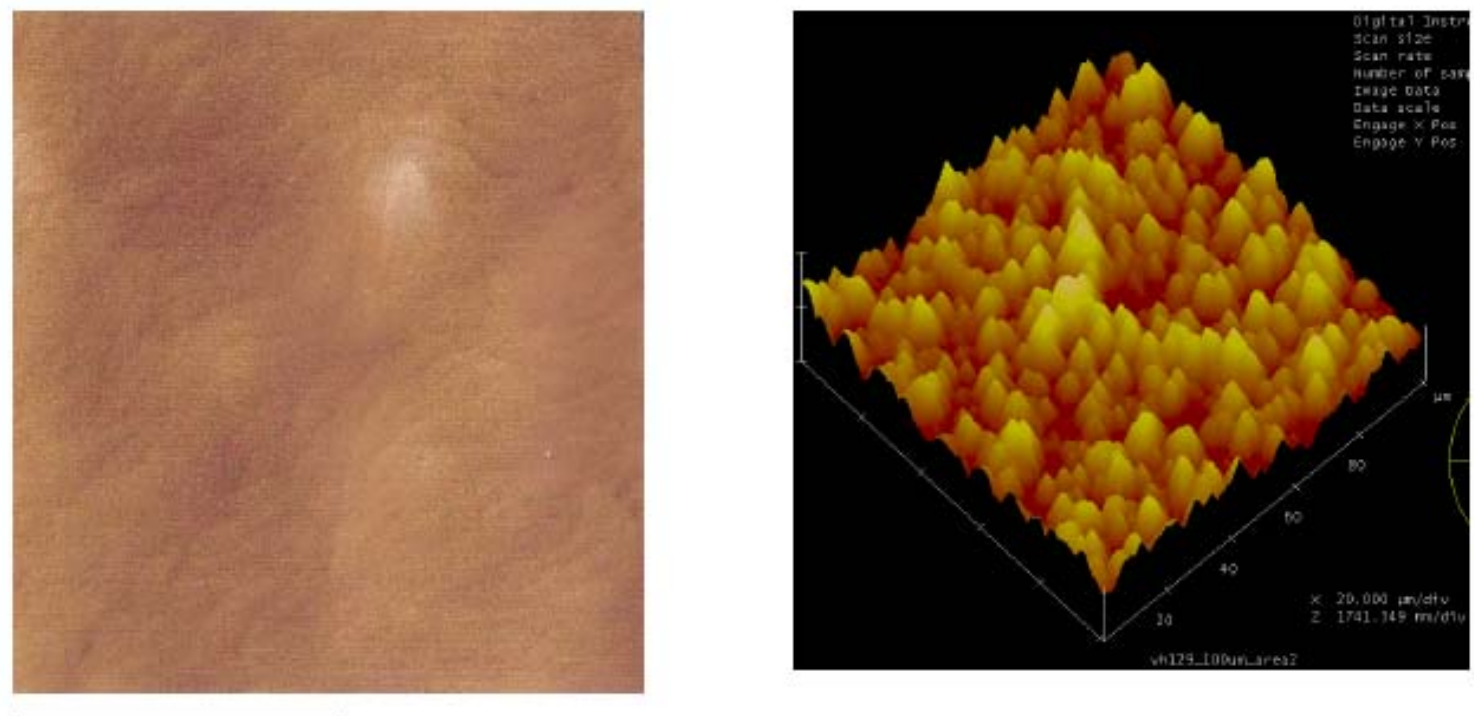

$5 \mu \mathrm{m}$

Figure 6: AFM images of an atomically smooth (0001) GaN template (left panel) and a spontaneously textured (0001) GaN template 


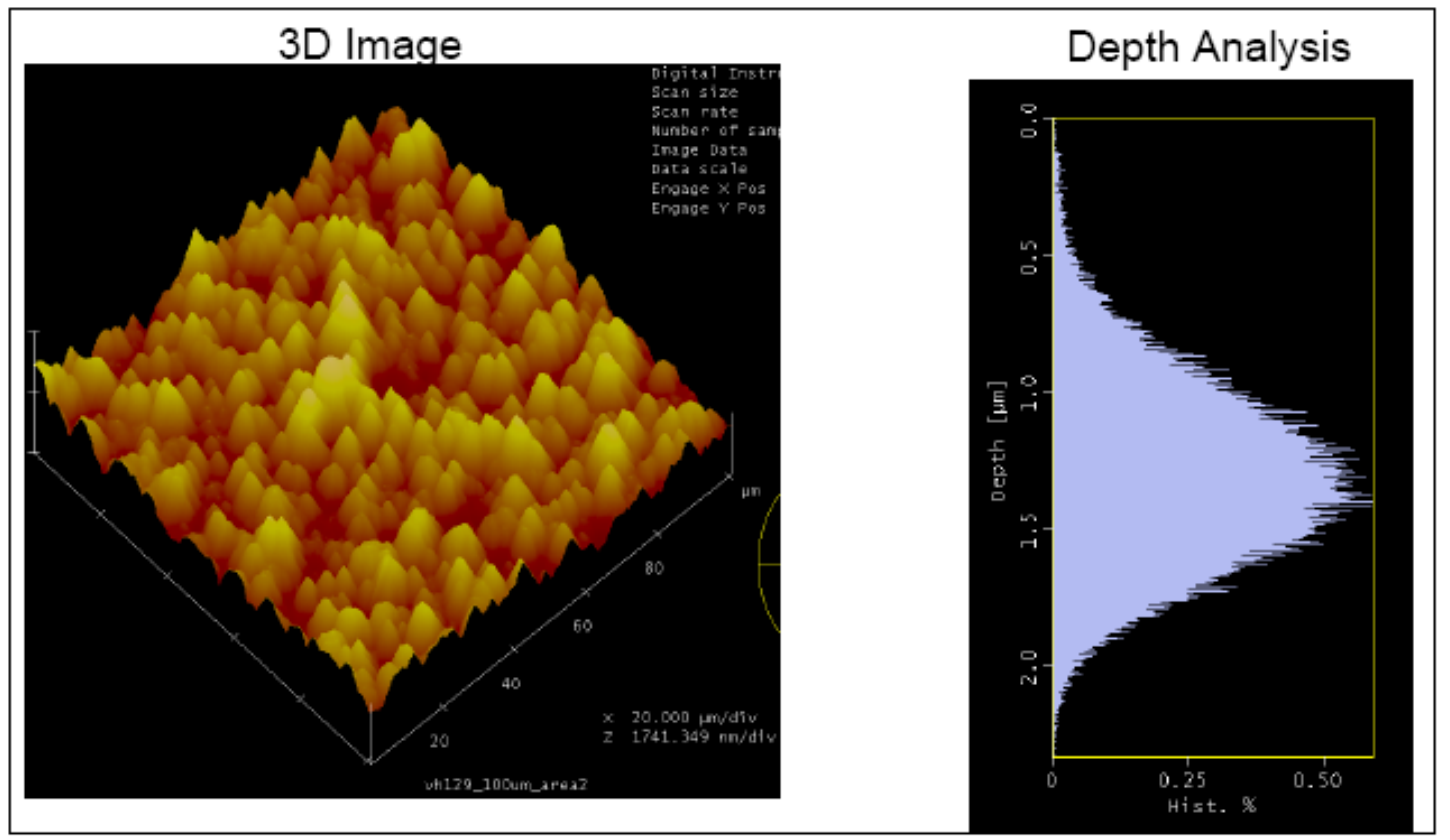

Figure 7: An AFM 3D image (left panel) and depth analysis of the same surface (right panel). The data clearly indicate that the depth distribution is a Gaussian distribution with an average depth of $1.3 \mu \mathrm{m}$.

\section{Surface Morphology of non-polar A-plane GaN, grown on the R-plane of sapphire}

As we reported previously [11] growth of $\mathrm{GaN}$ on the R-plane of sapphire leads to Aplane GaN. As shown in Figure 8, three unit cells of A-plane of GaN fit in one unit cell of R plane sapphire. Furthermore, under thermodynamic conditions of growth the Aplane (11-20) of $\mathrm{GaN}$ is terminated by the two (10-10) prism planes.

Figure 9 shows the surface morphology of a GaN template grown by the HVPE method on the R-plane sapphire. This surface morphology is consistent with the epitaxial relationship of the A-plane $\mathrm{GaN}$ and R-plane sapphire as discussed in Figure 8 


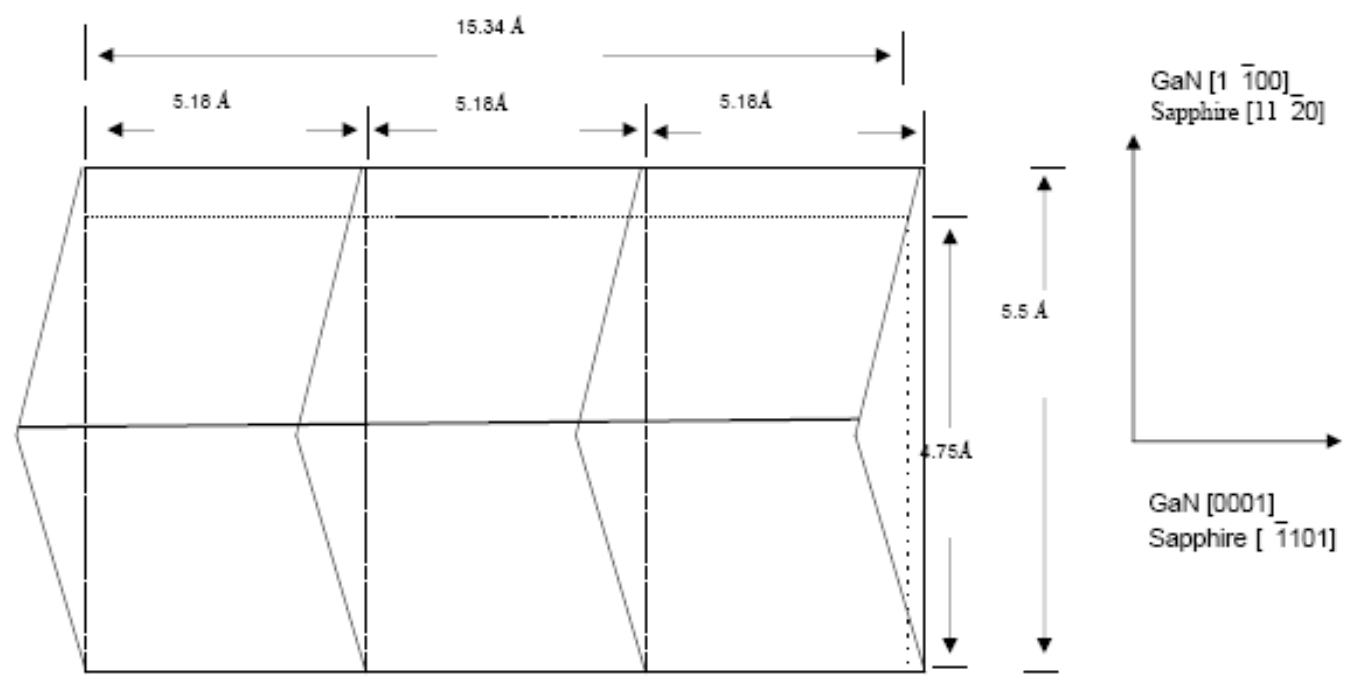

Figure 8: Epitaxial growth of non-polar (11-20) GaN on the R-plane sapphire.
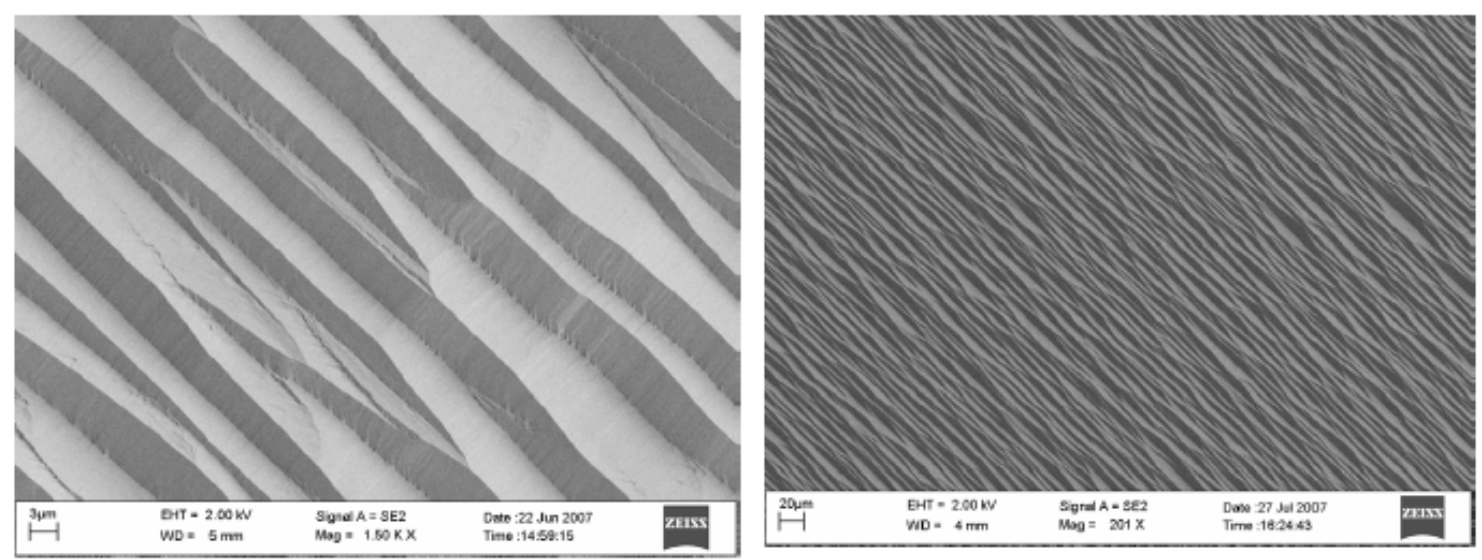

Figure 9: SEM Surface morphology in two different magnifications of non-polar (11-20) GaN grown by HVPE on the R-plane sapphire. 


\section{E. Characterization of the GaN Templates grown by the HVPE method}

\section{E. 1 Structure}

XRD measurements were performed to assess the crystalline quality of HVPE-grown GaN films in this work. Owing to the initial Volmer-Weber growth mode during nucleation of highly lattice mismatched $\mathrm{GaN}$ on sapphire, epitaxial GaN layers have a mosaic structure consisting of singlecrystal sub-grains with slight misorientations with respect to one another. Misorientation of these grains with respect to the C-plane is termed the tilt and the in-plane misorientation of the grains with respect to each other is termed the twist. The concepts of tilt and twist are illustrated in Figure 10.

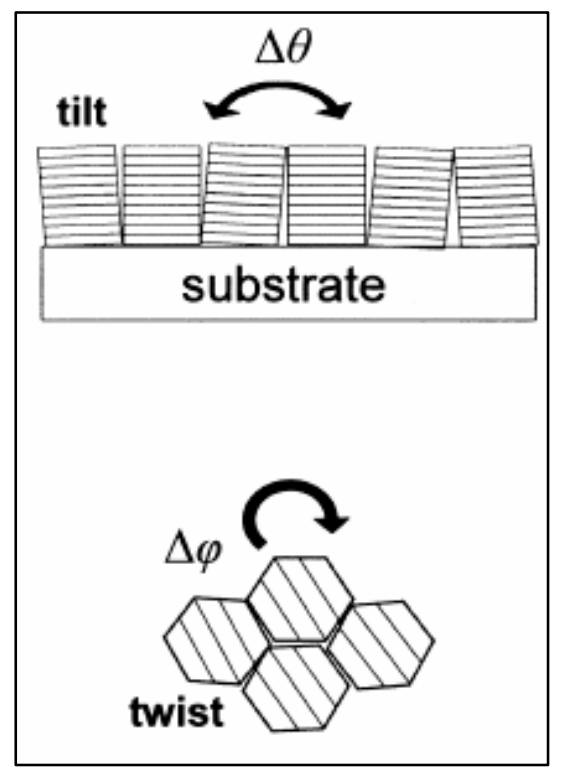

Figure 10: Schematic illustration of the misorientation of GaN grains with respect to each other during growth on sapphire.

The on-axis (002) rocking curve as well as two off axis rocking curves corresponding to (102) and (201) planes were examined in this study. From a qualitative standpoint, an assessment of the relative structural quality between two samples can be made by judging the narrowness of the rocking curves of various planes in each. Both on- and off-axis planes where chosen due to the fact that different defects present themselves in different planes. An example of this is the lack of contribution of edge dislocations to the broadening of on-axis (002) rocking curves, but yield contribution to the broadening of off-axis peaks. The distribution of tilt is associated with screw dislocations and is measured directly by (002) rocking curves. The distribution of twist is associated with edge dislocations and is measured indirectly by extrapolating the FWHM of planes at an inclination angle of $90^{\circ}$ with respect to the C-plane using planes of intermediate inclination angles. In this work, the (102) and (201) planes were observed, with inclination angles of $43.2^{\circ}$ and $75.1^{\circ}$ respectively, and used to extrapolate the twist component.

Figure 11 shows the FWHM of X-ray rocking curves of three planes for an MBE sample grown directly on sapphire, $5 \mu \mathrm{m}$ HVPE sample on sapphire, and a $400 \mu \mathrm{m}$ freestanding HVPE $\mathrm{GaN}$ sample. Table I summarizes this data. A progressive reduction of the twist and tilt 
distribution is observed. The difference in the structural quality of GaN grown by MBE versus HVPE samples can readily be attributed to the difference in the growth temperature employed by these methods. The higher temperature growth by HVPE leads to larger single crystal grain size, which translates into lower dislocation densities by virtue of the reduced number of grain boundaries. The difference between the quality of the $5 \mu \mathrm{m}$ and $400 \mu \mathrm{m}$ samples is attributed to dislocation annihilation processes that occur as $\mathrm{GaN}$ films are grown thicker.

There are a variety of sources that contribute to the broadening of the rocking curves. These include wafer curvature broadening, instrumentation broadening, dislocation broadening due to strain fields, and intrinsic crystal broadening. Theoretically, it is possible to extract dislocation densities from the broadening of rocking curves using a model proposed by Ayers taking these various components into account. However, there remain some issues in the application of this model to GaN due to analytical shortcomings such as the lack of account for planar defects such as stacking faults in the theory, which skew the results. There tends to be a systematic undercounting of dislocations by this method and was therefore not employed to determine dislocation densities in these studies.

Table I - Summary of XRD rocking curve FWHMs displayed in Figure 11

\begin{tabular}{|l|c|c|c|c|}
\hline & $\mathbf{( 0 0 2 )}-$ Tilt & $\mathbf{( 1 0 2 )}$ & $\mathbf{( 2 0 1 )}$ & Twist \\
\hline $1 \mu \mathrm{m}$ MBE & 600 & 1900 & 2400 & 2570 \\
\hline $5 \mu \mathrm{m}$ HVPE & 306 & 495 & 647 & 718 \\
\hline $400 \mu \mathrm{m}$ HVPE & 104 & 111 & 126 & 132 \\
\hline
\end{tabular}




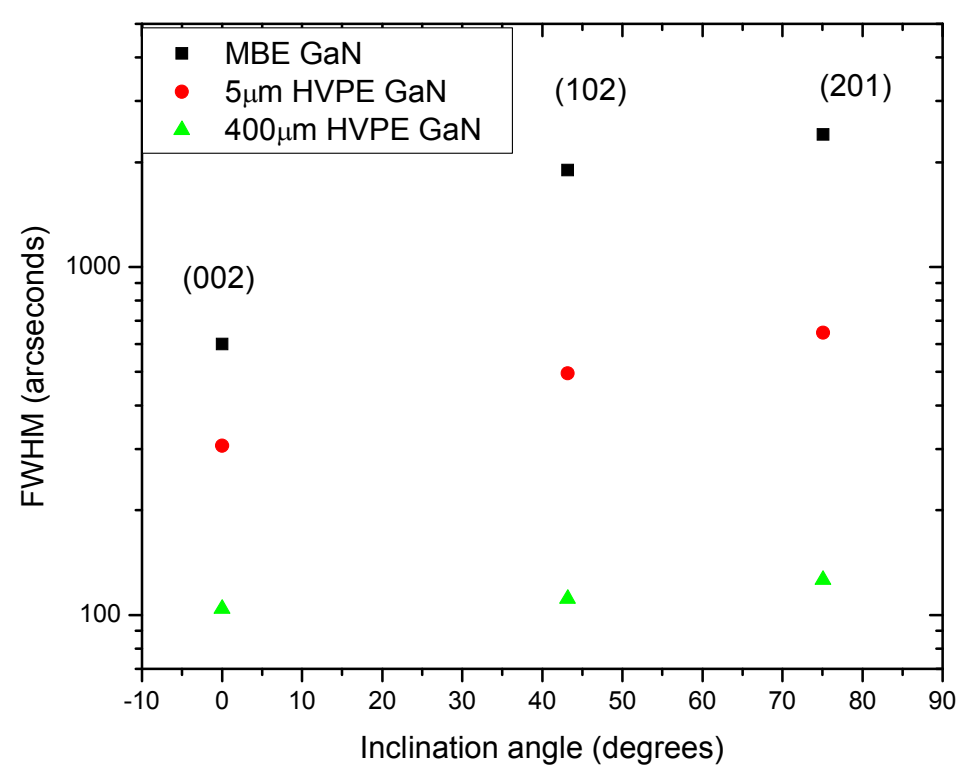

Figure 11: FWHM of X-ray rocking curves for on-axis and off-axis planes for the GaN samples described in the figure legend.

\section{E.2 Etch Pit Density Measurements}

A rapid characterization method for determining dislocation densities in $\mathrm{GaN}$ films is by measuring surface etch pit densities. Chemically etching a GaN surface under appropriate conditions can delineate dislocations due to the different electrical activity in the vicinity of dislocations that modify the local etch rate. Researchers have explored etching GaN in various wet chemistries in an effort to derive a quick method to accurately count dislocations without using a time and labor intensive method such as TEM. One such method that has been correlated well to TEM results is etching in phosphoric acid at $160^{\circ} \mathrm{C}$ for six minutes.

Various HVPE-grown GaN samples, ranging in thickness from $2.75 \mu \mathrm{m}$ to $1100 \mu \mathrm{m}$, were etched in $\mathrm{H}_{3} \mathrm{PO}_{4}$ under conditions just described. Figure 12 shows a GaN surface both before and after the etch procedure. It also shows the demarcation of etch pits for the purpose of counting. Figure 13 shows the resulting etch pit density measurements as a function of film thickness. A reduction of the etch pit density by two orders of magnitude is observed in the $1000 \mu \mathrm{m}$ film, with a value of $5 \times 10^{6}$ pits $/ \mathrm{cm}^{2}$ compared to $5 \times 10^{8} \mathrm{pits} / \mathrm{cm}^{2}$ for the $2.75 \mu \mathrm{m}$ film. 

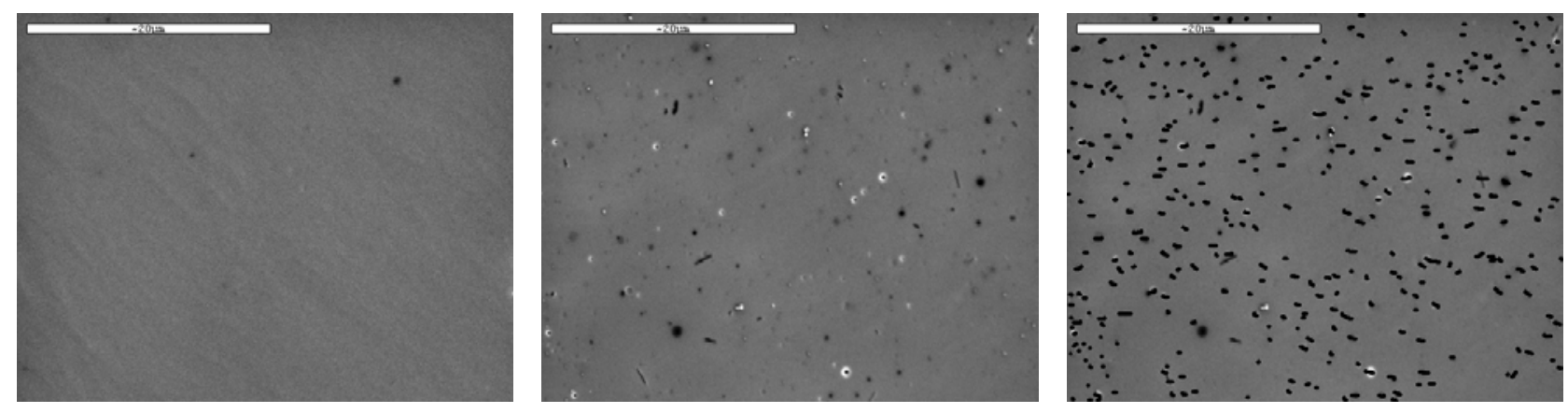

Figure 12 - (a) As-grown $\mathrm{GaN}$ surface, (b) $\mathrm{GaN}$ surface after $\mathrm{H}_{3} \mathrm{PO}_{4}$ etch as described in the text, (c) pits from the middle image are marked and counted to determine EPD.

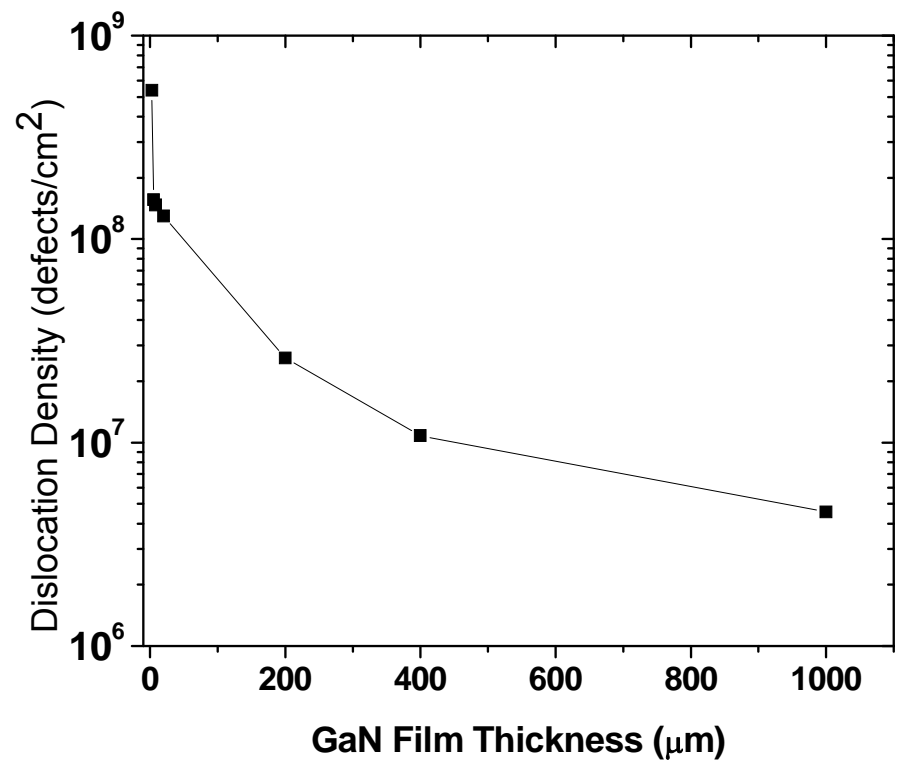

Figure 13: Dislocation densities, determined from EPD measurements, as function of GaN film thickness

\section{E.3 Electrical Transport Properties}

Hall effect measurements were carried out on a variety of HVPE GaN samples and their mobilities were studied as a function of their carrier concentration. Such measurements are plotted in Figure 14. The majority of thin films grown for this work were found to be degenerate with n-type conductivity. The most likely cause for this is the presence of unintentional dopants that enter the reactor with the gasses from the cylinders, the outgassing of chamber walls, and/or potential atmospheric leaks in the system. The presence of such dopants within the material leads 
to a large degree of impurity scattering, which limits the velocity of carriers. The freestanding samples were found to have lower carrier concentration, potential due to the longer growth times, which leads to the coating of the reactor walls and limits outgassing of impurities. The sample exhibiting the highest mobility is a freestanding sample with a carrier concentration of $1 \times 10^{17} / \mathrm{cm}^{3}$ with a mobility of $550 \mathrm{~cm}^{2} \mathrm{~V}^{-1} \mathrm{~s}^{-1}$.

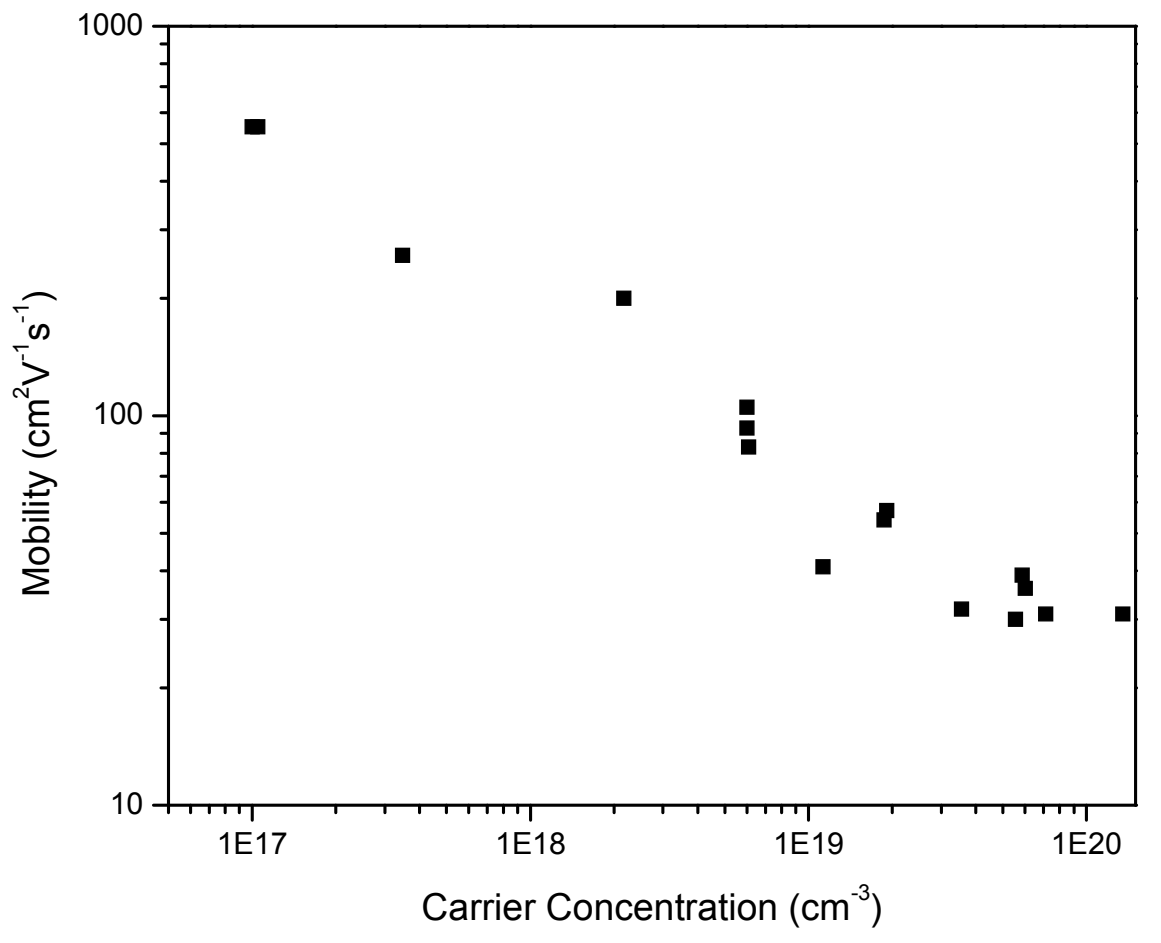

Figure 14: Mobility versus the carrier concentration of for a number of GaN films grown by HVPE

\section{F. Photoluminescence Properties}

Figure 15 shows the dependence of the photoluminescence as a function excitation for (0001) GaN templates with various degrees of texture. In all cases the Photoluminescence intensity varies linearly with light intensity indicating that the recombination is excitonic. Furthermore, the PL intensity increases by about a factor of 50 between the atomically smooth and the most textured GaN template. If we assume that the textured templates have about the same concentration of non-radiative recombination centers, then the observed increase in PL intensity from the textured GaN templates must be related to the more efficient extraction of light from the textured surface. 

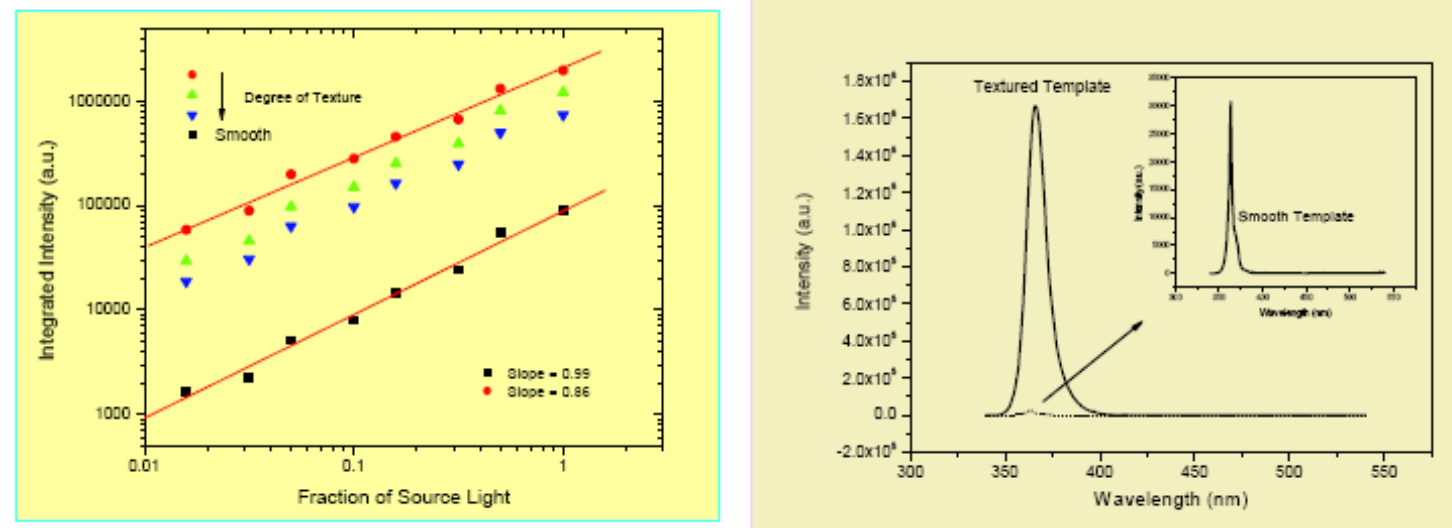

Figure 15: (Left panel) PL intensity as a function of excitation intensity for a number of GaN templates with different degree of texture. (Right panel) Comparison of the PL intensity of the smooth and the most textured GaN template. The inset shows the PL spectra of the smooth GaN template. The PL intensity of the textured GaN template is 50 times larger than that of the smooth GaN template.

\section{G. Summary of the results of Task 1.1}

Methods have been developed to deposit 50-100 $\mu \mathrm{m}$ thick GaN templates without cracks on the c-plane or the R-plane of sapphire The GaN templates on the c-plane sapphire can be made to be either atomically smooth (polar) or randomly textured (semi-polar). The GaN templates on the R-plane sapphire have the [11-20] orientation, are non-polar and are naturally textured.

The structure, the dislocation density, the transport and photoluminescence properties were measured and all indicate that these GaN templates are much less defective than films grown directly on the sapphire substrate by the MBE and MOCVD methods.

Films with growth rates as high as $700 \mu \mathrm{m} / \mathrm{hr}$ have been grown. Thus this method of growth is inherently inexpensive since the length of time for the growth of $\mathrm{GaN}$ templates 50-100 $\mu \mathrm{m}$ thick is about 5-10 min. Furthermore, the method uses about 1 liter / min of Ammonia compared to MOCVD, whose growth rates of 1-3 $\mu \mathrm{m} / \mathrm{hr}$, requires 10 liters / min of Ammonia. Those two factors contribute to the low cost of LEDs grown on such templates. 


\section{Task 1.2 Epitaxial Growth of $n$ - and p- GaN and AIGaN Layers by MBE and MOCVD on Textured GaN Templates}

Si-doped n-GaN and n-AlGaN films were grown on smooth and textured GaN templates. However, their doping level is difficult to evaluate because the GaN substrate is also n-type.

Mg-doped p-GaN films grown on smooth and textured GaN templates and the results are shown in Figure 16. As seen from these data the samples can be doped p-type with carrier concentration in excess of $10^{18} \mathrm{~cm}^{-3}$. Furthermore, lightly doped samples were found to have mobilities larger than $100 \mathrm{~cm}^{2} / \mathrm{V}$.s.
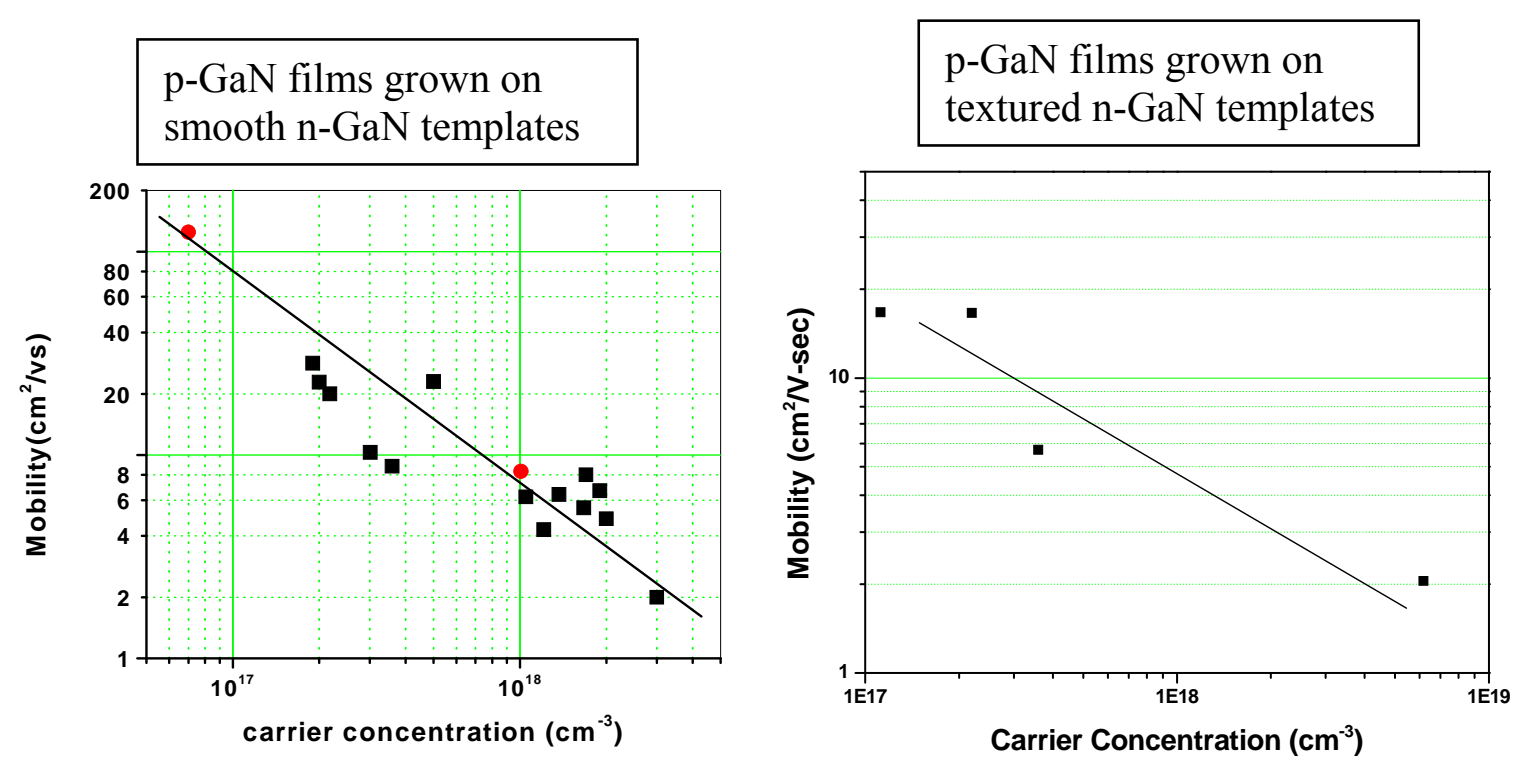

Figure 16: Mobility vs hole concentration for $\mathrm{Mg}$ doped $\mathrm{P}-\mathrm{GaN}$ films grown on smooth (left panel) or textured HVPE (0001) GaN templates

Despite of the great need of p-AlGaN for III-N devices, it is difficult to achieve high conductivity in p-type AlGaN alloys for two main reasons. First, the activation energy of $\mathrm{Mg}$ in $\mathrm{Al}_{\mathrm{x}} \mathrm{Ga}_{1-\mathrm{x}} \mathrm{N}$ increases with the AlN mole fractions. For example, the value of Ea estimated from the effective mass theory for $\mathrm{x}=0.25$ is between $263 \mathrm{meV}$ and $294 \mathrm{meV}$. The free hole concentration in a semiconductor with acceptor concentration $N_{A}$ and acceptor binding energy $E a$ is given by: 


$$
p=\sqrt{\frac{1}{g} N_{A} N_{V}} \exp \left(-\frac{E_{a}}{2 k T}\right)
$$

Where $g$ is the acceptor degeneracy, $N_{V}$ is the effective density of states at the valence band edge, and $k T$ is the thermal energy. For an acceptor energy of $280 \mathrm{meV}$ and acceptor concentration of $1 \times 10^{18} \mathrm{~cm}^{-3}$, the electrical activation calculated is only $0.27 \%$ at room temperature.

Another difficulty is that the $\mathrm{O}$ donor concentration will increase with increasing AlN mole fraction due to the strong affinity between $\mathrm{O}$ and $\mathrm{Al}$, resulting in insulating or even n-type characteristics despite of heavy $\mathrm{Mg}$ doping. Oxygen is a shallow n-type donor with activation energy $\sim 29 \mathrm{meV}$ in the nitride system and is suspected to be at least partially responsible for the unintentionally doped n-type behavior of the nitrides in addition to forming DX like states in AlGaN compounds.

Figure 17 shows our results of doping AlGaN alloys with 25\% AlN mole fraction as a function of Ga flux during growth by plasma assisted MBE. We notice that the most conductive films are grown under Ga-rich conditions of growth. This is in agreement with our findings that $\mathrm{GaN}$ dopes more efficiently p-type under Ga-rich conditions [12]. This result was interpreted that the $\mathrm{Mg}$ which has a very high vapor pressure at the growth temperature dissolves in the $\mathrm{Ga}$ and incorporates into the GaN network from the liquid phase.

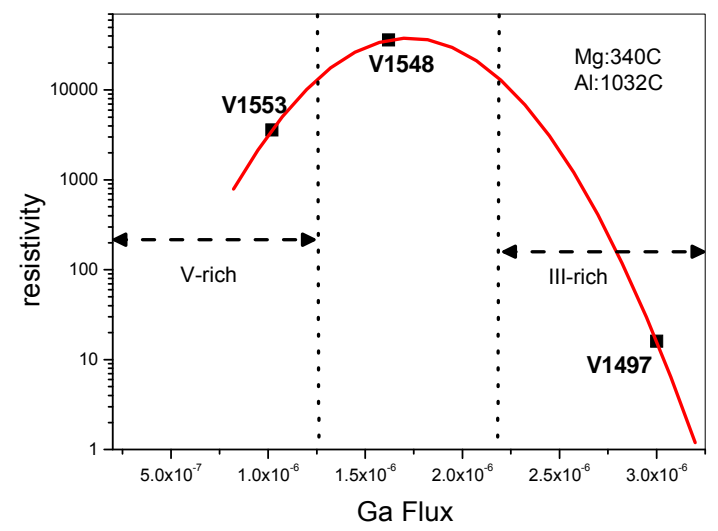

Figure 17: Resistivity vs Ga flux for p-AlGaN with $25 \%$ AlN mole fraction 


\section{Task 1.3 Epitaxial growth of InGaN / AlGaN single and Multiple Quantum Wells by MBE and MOCVD on Textured GaN Templates}

Summary: In this section we discuss our effort in developing Nitride multiple QWs emitting in the UV and visible parts of the electromagnetic spectrum

- Development of AlGaN/GaN MQWs emitting in the wavelength range 350$360 \mathrm{~nm}$

- Development of InN/GaN MQWs emitting in the wavelength range 380-400nm. Monolayer of InN grows coherently on $\mathrm{GaN}$ (no misfit dislocations). Since $\mathrm{InN}$ is under compressive stress, it can be deposited at temperatures compatible with that of GaN. Such MQWs are appropriate for LEDs used for the excitation of tricolor phosphor

- Development of InGaN/GaN MQWs emitting in the wavelength range 400$550 \mathrm{~nm}$

\section{A. (0001) GaN / AlGaN MQWs emitting in the 350-360 nm.}

III- Nitride MQWs are used as the active region of visible and UV LEDs and lasers, as well as for other devices such as optical modulators and devices based on intersubband absorption. For LED devices it is very important to optimize the MQW active region in order to improve the internal quantum efficiency (IQE) and possibly the light extraction efficiency. The IQE, during optical pumping of the MQWs, is the ratio of the number of generated photons inside the semiconductor to the number of optically injected electron-hole pairs. This parameter depends strongly on a number of factors such as point defects and dislocations. These defects, and in particular those generating energy states close to the middle of the energy gap, act as nonradiative recombination centers. Furthermore, Auger processes, occurring in highly doped directbandgap semiconductors, also contribute to non-radiative recombination processes. Another factor that influences the IQE is the operating temperature. All of these factors influence the IQE in QWs based on traditional semiconductors such as GaAs, which have cubic symmetry.

For semiconductors having the wurtzite structure such as III-Nitrides, polarization effects also play a role in reducing the internal quantum efficiency. Specifically, in MQWs grown along the polar [0001] or [0001] directions, there are internal electric fields present across the QWs and barriers due to spontaneous and piezoelectric polarization. These internal electric fields distort the QWs and barriers, a phenomenon known as the quantum-confined Stark effect (QCSE). The QCSE affects the emission properties of the QWs in two different ways. First, the emission wavelength is red- shifted, because the energy difference between the ground state for holes and electrons becomes smaller. Second, the PL intensity is expected to be reduced because the electron and hole wavefunctions are physically separated, and thus the excitonic oscillator 
strength is reduced. Our group has previously reported the emission properties of GaN/AlGaN MQWs grown on the $c$-plane (polar) and the m-plane (non-polar) GaN templates [13]. The emission from the MQWs grown on the $c$-plane was found to be red-shifted and to have significantly reduced luminescence efficiency compared to those grown on the $m$-plane. This reduction of luminescence intensity is higher if the quantum wells are wider than $3 \mathrm{~nm}$, since the distortion for narrower QWs does not affect as much the overlap of the wavefunctions of the electrons and holes. For this reason, Nitride LEDs grown along the polar [0001] direction employ relatively narrow quantum wells.

A number of approaches have been proposed to enhance the extraction of light from LEDs. These include, for example, epitaxial lift-off and wafer-bonding methods to transfer GaAs LEDs to transparent substrates to eliminate absorption of the emitted light by the GaAs substrate. Other approaches involve either the use of transparent material with continuously variable index of refraction to reduce back-reflection at the interface or micro-texturing the extraction surface of the LED structure lithographically. However, none of these approaches relies upon modification of the QWs in order to improve the light extraction efficiency.

In here, we report on the PL properties of GaN/AlGaN MQWs grown on atomically smooth and randomly textured (0001) GaN templates. We find that the PL intensity from the textured MQWs is orders of magnitude higher than that obtained from identically formed smooth MQWs, a result attributed to the combined effect of enhanced internal quantum efficiency and light extraction efficiency.

GaN/AlGaN MQWs were grown by MBE on both smooth and textured GaN templates and were found to replicate the GaN templates. The formation of the MQWs on the textured GaN surface was confirmed both by cross-section TEM studies as well as high-resolution X-ray diffraction (XRD) measurements. Figure 18 shows a cross-section electron micrograph and Figure 19 shows the X-ray diffraction pattern around the (0002) Bragg peak for a ten-period GaN $(7 \mathrm{~nm}) / \mathrm{Al}_{0.20} \mathrm{Ga}_{0.8} \mathrm{~N}(8 \mathrm{~nm}) \mathrm{MQWs}$. Fig. 19 also shows a simulation of the XRD result using the kinematical scattering model. From the simulation, we determined that the period of the MQWs is $15.4 \mathrm{~nm}$, in good agreement with the designed thicknesses of 8-nm-barrier and 7-nmwell widths. From the position of the zeroth order superlattice peak (assuming the validity of Vegard's law in this material system), we determine the AlN mole fraction in the AlGaN barriers to be $\sim 20 \%$, also in agreement with the designed Al-composition. It is important to note in Figure 18 that the thicknesses of the QWs in the inclined surfaces are thinner than those in the (0001) planes. 


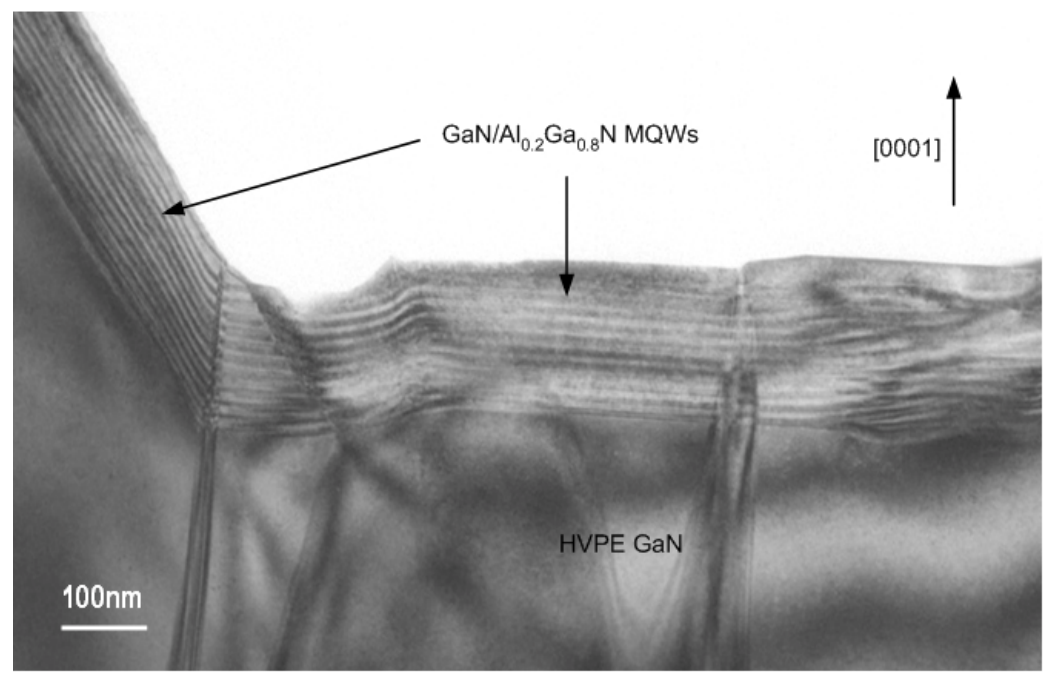

Figure 18: Cross-section electron micrograph of 10 pairs of $\mathrm{GaN}(7 \mathrm{~nm}) / \mathrm{Al}_{0.2} \mathrm{Ga}_{0.8} \mathrm{~N}(8 \mathrm{~nm})$ MQWs grown on textured GaN templates. The image shows that the textured MQWs have coated the substrate surface in a conformal way. These data also show that the MQWs are thinner on the sloping surface.

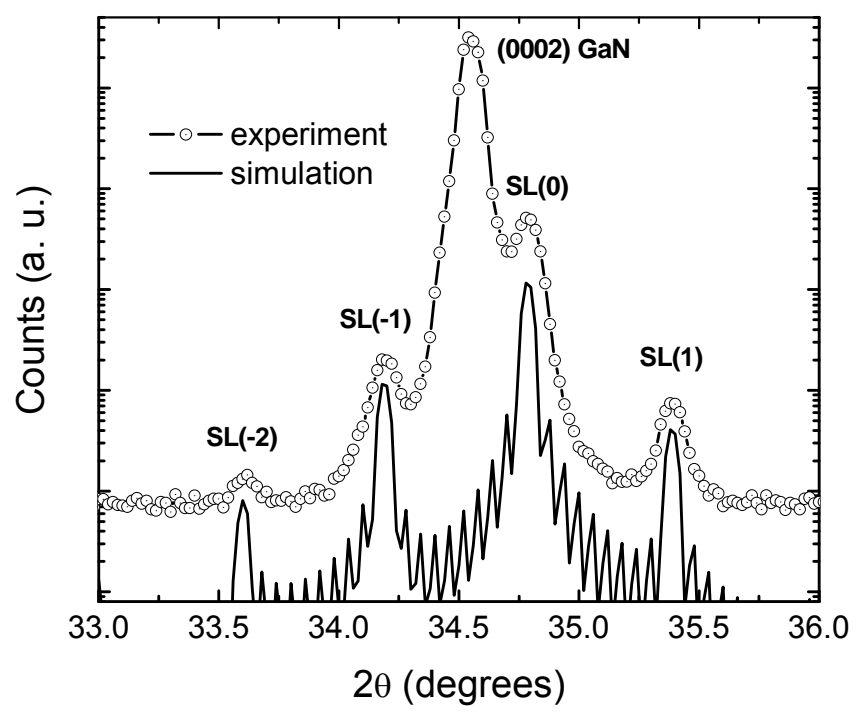

Figure 19: X-ray diffraction pattern around the (0002) Bragg peak for a ten-period $\mathrm{GaN}(7 \mathrm{~nm}) /$ $\mathrm{Al}_{0.20} \mathrm{Ga}_{0.8} \mathrm{~N}(8 \mathrm{~nm})$ MQWs. Shown also is the simulation of the XRD data using the kinematical scattering model.

Figure 20 shows the PL spectra of $\mathrm{GaN}(7 \mathrm{~nm}) / \mathrm{Al}_{0.20} \mathrm{Ga}_{0.8} \mathrm{~N}(8 \mathrm{~nm}) \mathrm{MQWs}$ grown on smooth and textured $\mathrm{GaN}$ templates. The spectra were measured at $300 \mathrm{~K}$ under identical conditions and plotted on the same scale for comparison. The PL spectra from the MQWs grown on the smooth GaN template show only a small peak at $364 \mathrm{~nm}$ which is attributed to luminescence emitted from the bulk GaN substrate. The PL peak from the MQWs should be 
red-shifted with respect to the bulk GaN luminescence and should be reduced significantly in magnitude due to QCSE. To resolve the emission from the MQWs, we measured the cathodoluminescence (CL) spectra of the same sample. During CL measurements, a very low acceleration voltage $(4 \mathrm{kV})$ was used to ensure that the sample is probed near the surface. The inset in Fig. 20 shows the CL spectra of the same sample. A prominent peak is visible at $364 \mathrm{~nm}$ corresponding to luminescence from the GaN substrate and a weaker emission at $396 \mathrm{~nm}$, which corresponds to emission from the MQWs. The stronger CL emission from the QWs compared to that of PL is attributed to the higher excitation level by the electron beam, since each electron generates multiple electron-hole pairs. Thus, luminescence from the MQWs is red-shifted with respect to that of the bulk GaN emission. This red-shift, as well as the weak luminescence, is qualitatively consistent with the QCSE, as discussed previously. On the contrary, the spectrum from the textured MWQs shows a broad peak centered around $359 \mathrm{~nm}$. This peak is blue-shifted with respect to bulk GaN emission and its origin is PL from the MQWs. Thus the emission spectra from the textured MQWs show exactly the opposite trend from those of the smooth MQWs. These results are consistent with the notion that there is no significant distortion in the shape of the QWs due to internal fields, since the majority of the textured QWs planes are not normal to the [0001] polarization direction.

From the data of Fig. 20, we see that the peak PL intensity of the textured MQWs is more than 700 times higher than the PL peak intensity from the smooth MQWs. This result can be accounted for by comparing the extraction efficiency from a smooth and a randomly textured $\mathrm{GaN} / \mathrm{AlGaN}$ MQWs. Based on the large contrast between the $\mathrm{GaN}$ index of refraction $\left(\mathrm{n}_{\mathrm{o}}=2.5\right)$ and air $(n=1)$, the expected theoretical maximum fraction of extracted light is only $4 \%$ of the incident radiation. On the other hand, due to random texturing of the surface, there is an increase in the escape probability of a single photon since the escape cone is not limited to that defined only by the indices of refraction. The index of refraction in the textured surface varies gradually along the optical axis from the value of 2.5 to 1.0, i.e. there are additional escape angles available for each emitted photon due to random texture at the interface. This is analogous to transmittance through a diffraction grating, wherein the grating imparts a phase shift to the incident wave and bends the wave-front at specific angles depending on the wavelength of the incident light [14]. In this case, the phase shift is controlled by the periodic variation of thickness (or periodic "surface texture") of the grating material at the grating/air interface. In the case of the textured GaN/AlGaN MQWs, the texture of the surface is not periodic, but random, creating random phase shifts across the interface and leading to escape-angle randomization which effectively increases the photon escape probability. 


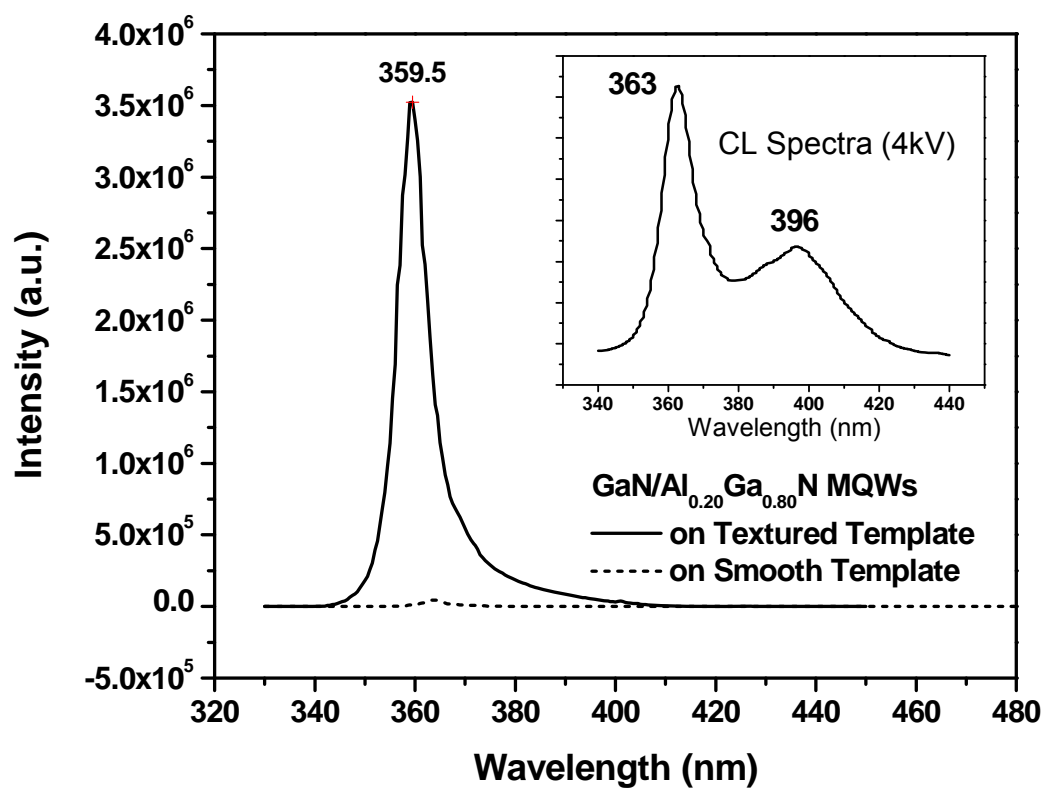

Figure 20: Comparison of the PL spectra between smooth MQWs and textured MQWs. The intensity of the PL peak of the textured MQWs is $>700$ times than that of the smooth MQWs. The inset shows the resolved luminescence peak from the smooth MQWs using low-voltage (4 $\mathrm{kV}) \mathrm{CL}$ measurements.

If we hypothesize that $100 \%$ of the generated light inside the textured MQWs is extracted through surface, then this can account for a factor of 25 of the observed enhancement in the luminescence. In reality, however, the extraction efficiency is likely to be less than $100 \%$ and thus the enhancement in luminescence due to extraction efficiency is a factor less than 25 . The rest of the enhancement in the PL intensity of the textured MQWs must be due to increase in internal quantum efficiency, as will be discussed below.

Since the QCSE is expected to depend on the width of the QWs, we also investigated the PL spectra of QWs with thickness $5.5 \mathrm{~nm}$. Figure 21 shows the dependence of the emission peak (Fig. 21(a)) and the luminescence intensity (Fig. 21(b)) versus well width for both smooth and "textured" $\mathrm{GaN} / \mathrm{Al}_{0.2} \mathrm{Ga}_{0.8} \mathrm{~N}$ MQWs. As seen in Fig. 21(a), the PL spectra from the smooth MQWs are red-shifted while those from the textured MQWs are slightly blue-shifted with respect to the bulk GaN emission. Correspondingly, the PL intensity from the smooth MQWs increases as the well width becomes narrower while there is only a slight increase from the textured MQWs. These results are qualitatively consistent with the QCSE. 

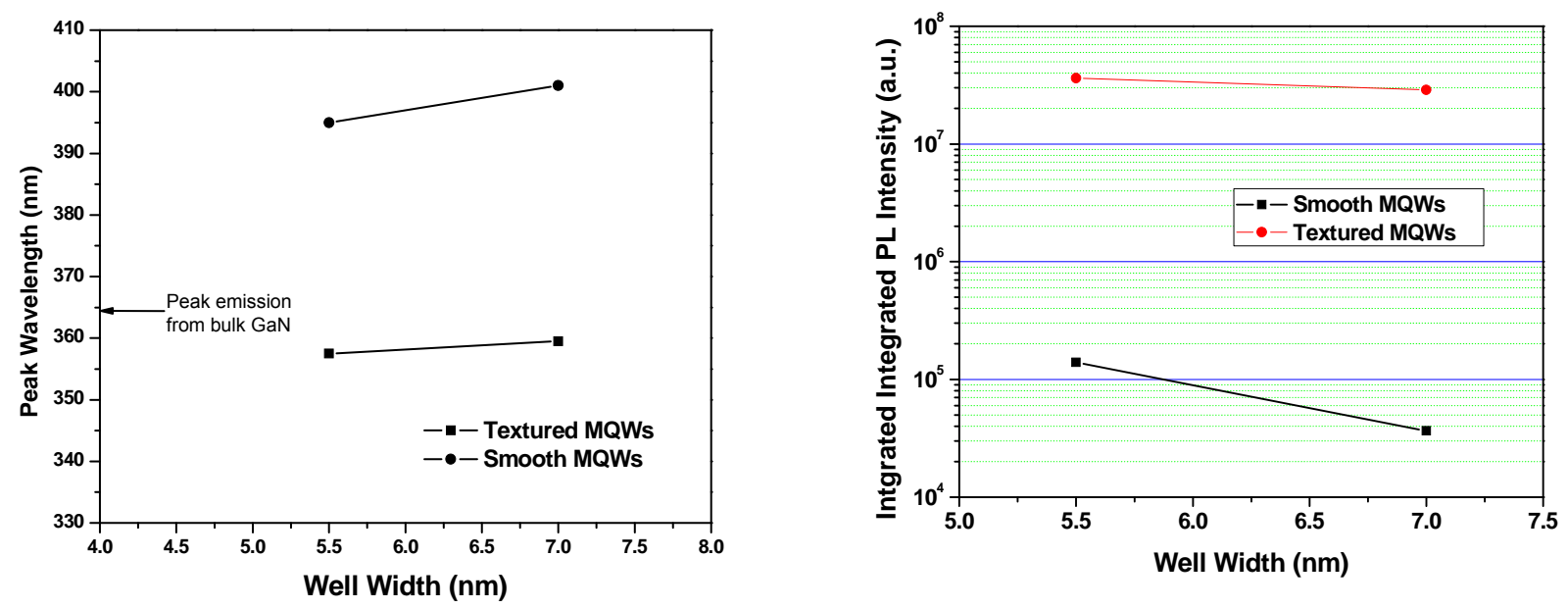

Figure 21: Plots of (a) PL peak position and (b) PL peak intensity versus well width for GaN / $\mathrm{Al}_{0.2} \mathrm{Ga}_{0.8} \mathrm{~N}$ MQWs grown on smooth and textured $\mathrm{GaN}$ templates.

To better understand the emission from the textured MQWs, low-voltage $(2 \mathrm{kV})$ spot CL (with surface excitation radius $\sim 0.03 \mu \mathrm{m}$ ) was done on different areas of the wafer containing the textured MQWs. Figure 22(a) shows a surface profile of the sample surface along an arbitrary direction obtained using AFM. Points A, B and C indicate where spot CL spectra were taken. For comparison, CL spectra from a large sample area $(60 \mu \mathrm{m} \times 40 \mu \mathrm{m})$ were also taken using the TV scan mode. These spectra are shown in Fig. 22(b) and consist of a major peak at $356 \mathrm{~nm}$ and a smaller one at $376 \mathrm{~nm}$. The major peak can be accounted for from emission from QWs whose planes are not normal to the [0001] polar direction (blue-shifted with respect to emission from bulk $\mathrm{GaN}$ ) and the second represents emission from QWs with planes that are almost normal to the [0001] direction (red-shifted with respect to emission from bulk GaN). Figure 22(c) shows the spot CL spectra taken at Point A. The data were fitted to two Lorentzian curves with peaks at $382 \mathrm{~nm}$ and $358 \mathrm{~nm}$. The first peak, which is red-shifted with respect to bulk GaN emission, is the dominant one because the planes of the QWs at point A are almost normal to the [0001] polar direction. The blue-shifted smaller peak at $358 \mathrm{~nm}$ probably originates from QW planes which are not normal to the [0001] direction since the electron beam surface excitation has finite dimensions. Figure 22(d) shows the luminescence spectra taken at Point B, where the growth surface is not perpendicular to the [0001] direction. Fitting of the data shows one stronger peak at $356 \mathrm{~nm}$, originating from the QWs, and a weaker one at $364 \mathrm{~nm}$, originating from the GaN template underneath the QWs. The peak at $364 \mathrm{~nm}$ is indicative of bulk luminescence which may be due to the e-beam penetrating in to the bulk through thinner QWs in this region of the sample because the growth rate along these slopes may be slower compared to the planar growth rate. Finally, Figure 22(e) shows the spot CL spectrum taken at Point C. This area is very close the point where the QW planes intersect. This spectrum show strong luminescence peak at $356 \mathrm{~nm}$. It is this CL peak that dominates the spectrum when the measurement is done using the TV mode (as seen in Fig. 22(b)). It appears therefore, that the spontaneous emission rate at Point $\mathrm{C}$ is significantly enhanced. 
While the enhancement of the PL intensity from QW planes, which are not normal to the [0001] direction, is qualitatively expected due to minimization of the QCSE, the mechanisms responsible for the enhancement in spontaneous emission at the wedges of the QWs is not well understood at this time. One potential mechanism is the transition in carrier behavior from 2D to 1D (and potentially 0D) due the $\mathrm{V}$-shaped intersecting planes of the quantum wells, meaning that the wedges are likely to behave as quantum wires (or quantum dots) causing localization and trapping of excitons. In addition, due to polarization component parallel to the QWs, as shown in Figure 23(a), one expects electron accumulation at the wedges, as shown in Fig. 23(b). Depending on the equilibrium charge density at these wedges, enhancement in spontaneous emission may result from plasmonic effects.

In conclusion we have demonstrated that GaN/ AlGaN MQWs grown on textured GaN templates replicate the random texture of the substrate. Their peak PL intensity from MQWs (well/barrier thickness of $7 \mathrm{~nm} / 8 \mathrm{~nm}$ ) is 700 times more intense compared to that from identically grown but smooth (0001) GaN/AlGaN MQWs. This significant enhancement in PL intensity is attributed partly to increase of light extraction efficiency and partly to increase in internal quantum efficiency due to suppression of the quantum-confined Stark effect. Additionally, charge redistribution and localization due to polarization effects may lead to additional enhancement of the spontaneous emission due to plasmonic effects.

These experiments show that textured MQWs grown on GaN textured templates are very promising for realizing GaN-based LEDs with very high photon conversion efficiencies. The use of textured MQWs on GaN textured templates not only enhances the light extraction efficiency, but also enhances the internal quantum efficiency of the QW structures. 

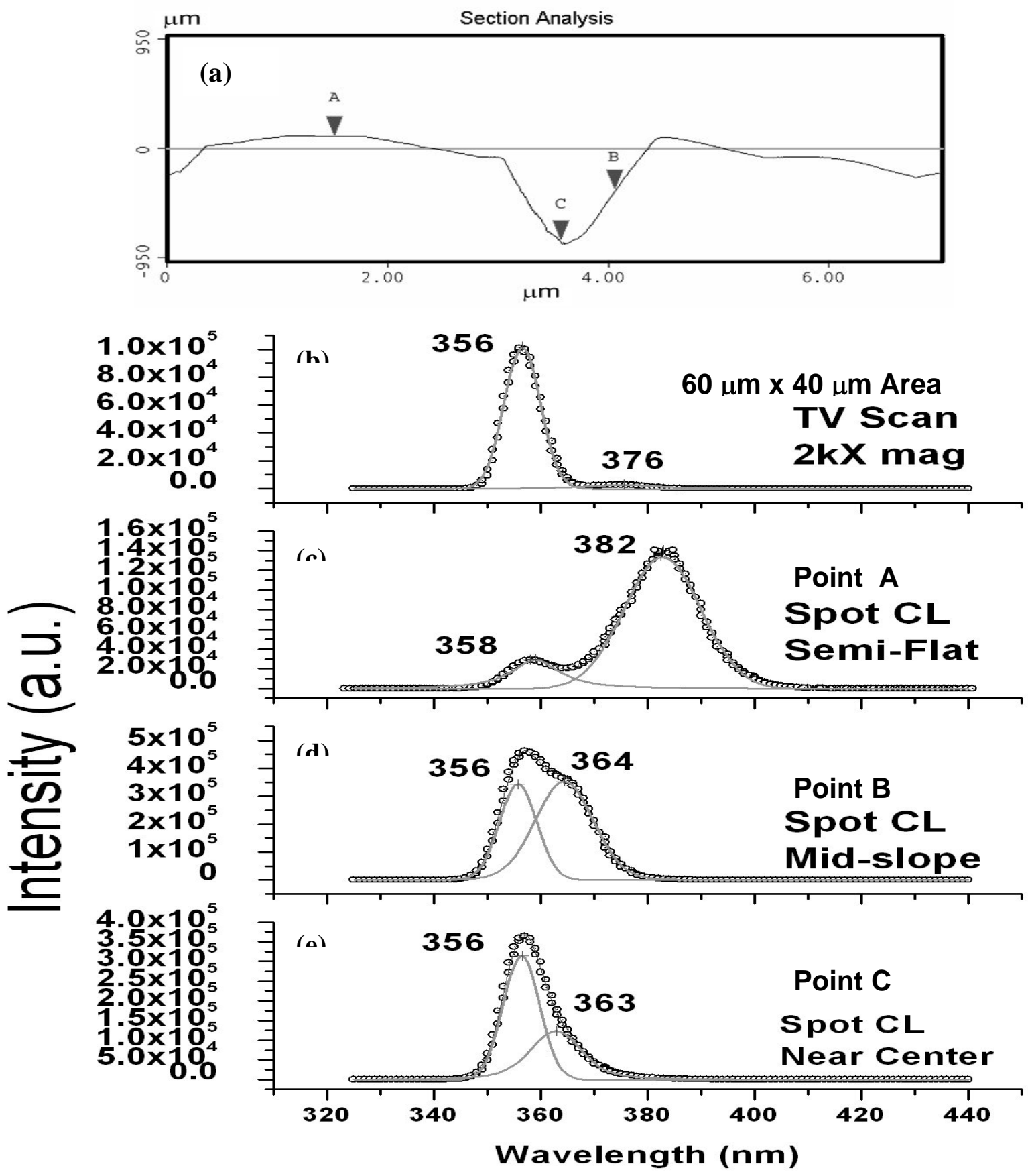

Figure 22: (a) AFM surface profile along an arbitrary direction of the textured MQWs. CL spectra were collected using $2 \mathrm{kV}$ acceleration voltage from a large area of the sample (b) as well as spot CL taken at points $\mathrm{A}, \mathrm{B}$ and $\mathrm{C}$. 

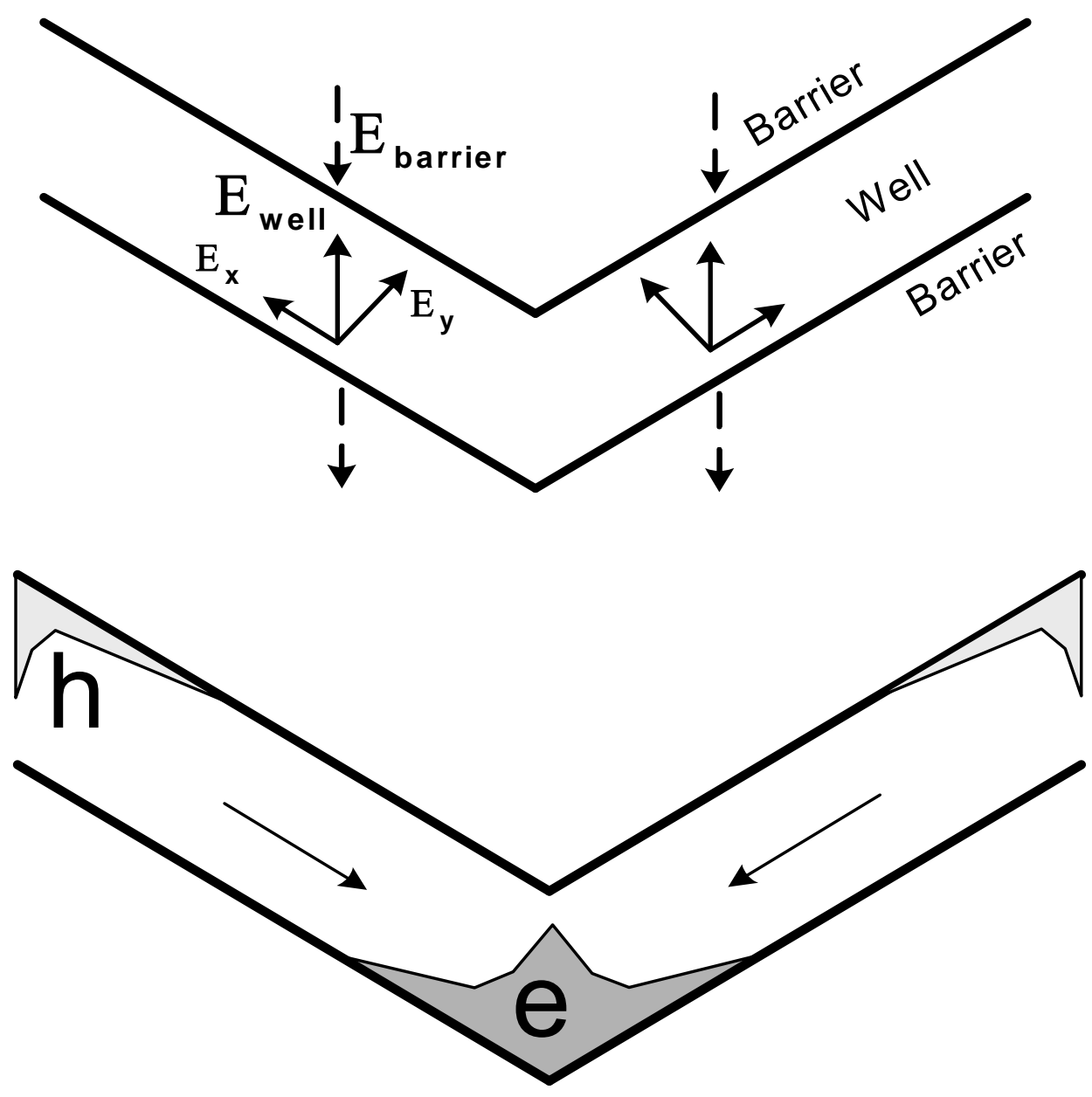

Figure 23: (Upper panel) Schematic illustration of the internal electric fields in wells and barriers arising from polarization effects. (Lower panel) Expected charge redistribution in QWs due to the electric field component parallel to the QW planes.

To confirm that textured QWs have higher IQE we measured this parameter directly by studying the PL intensity as a function of temperature. The definition of the IQE at room temperature is given by the expression:

\section{$\mathrm{IQE}=\left(\mathrm{PL}\right.$ Intensity at $\left.300^{\circ} \mathrm{K}\right) /\left(\mathrm{PL}\right.$ Intensity at $\left.10^{\circ} \mathrm{K}\right)$}

This definition assumes that the recombination at $10 \mathrm{~K}$ is radiative due to localization of the excitons at that temperature.

Figure 24 shows the PL intensity for smooth GaN / AlGaN MQWs as well as two others with various degrees of roughness. Thus, we have measured directly that the IQE increased by more than a factor of 20 between the smooth and the most textured QWs. This direct measurement is in qualitative agreement with the conclusions we have arrived earlier. 


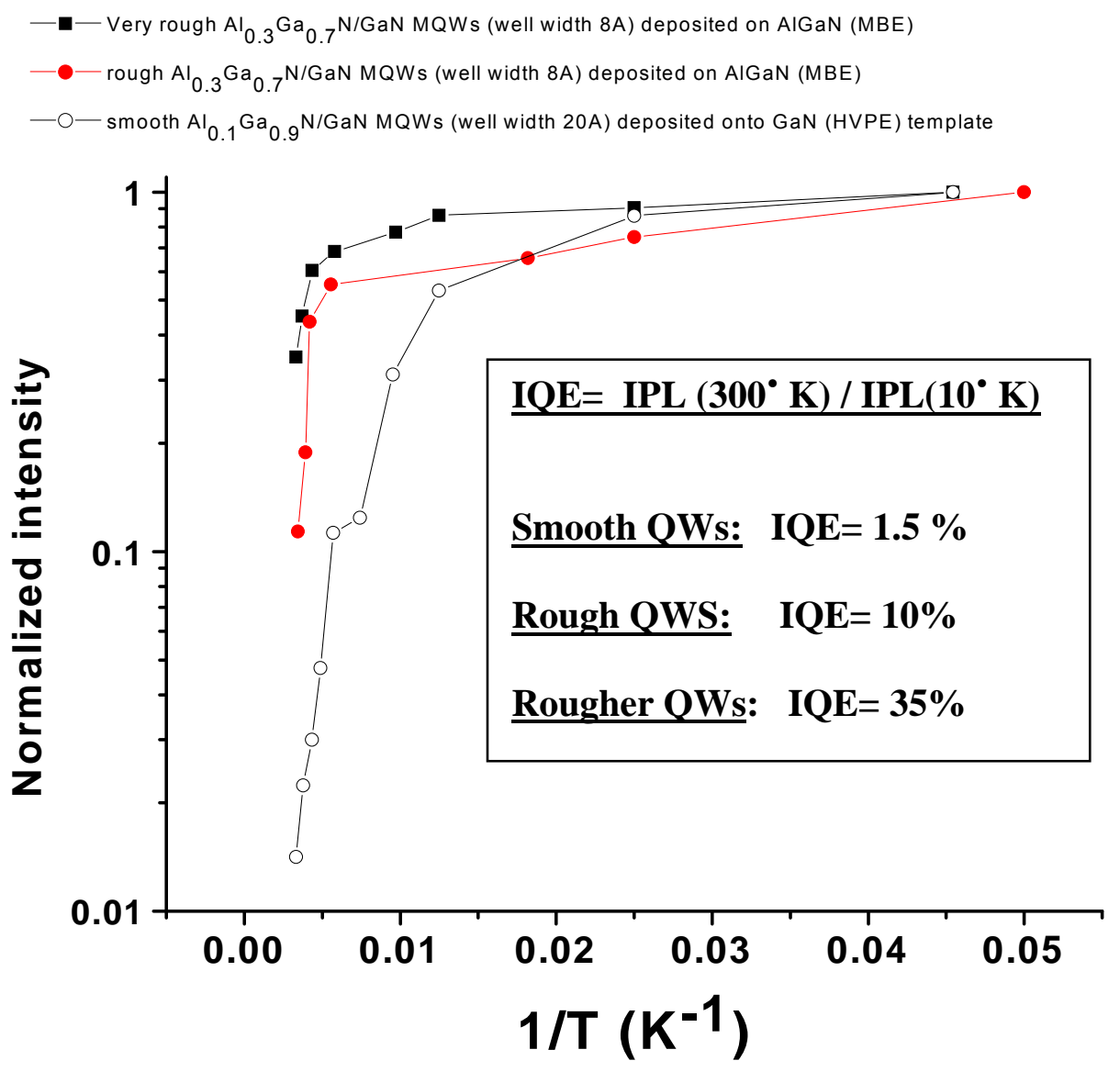

Figure 24: PL intensity as a function of temperature for GaN /AlGaN MQWs with various degrees of texture.

\section{B. Near UV LEDs based on monolayer InN wells and thicker GaN barriers}

Because the InGaN alloys tend to undergo phase separation and atomic ordering our group has developed InN / GaN MQWs. If we take into account that the lattice mismatch between $\mathrm{InN}$ and $\mathrm{GaN}$ is $11 \%$ one can calculate that the critical thickness of $\mathrm{InN}$ for coherent growth on $\mathrm{GaN}$ is one monolayer. The monolayer of $\mathrm{InN}$ will be under extreme compressive stress and thus, its melting point is expected to be significantly higher than that of the bulk InN. Due to that the monolayer of $\mathrm{InN}$ can be deposited at higher temperatures $\left(700{ }^{\circ} \mathrm{C}\right)$ than its normal temperature below $500{ }^{\circ} \mathrm{C}$. Such high 
temperature of growth should lead to InN with better structural quality. Based on these arguments one expects the following benefits by using such InN/GaN MQWs as the active region of the LED structures:

- LEDs based on coherent InN / GaN MQWs where the InN is a single monolayer are expected to be very efficient since the quantum wells will be free of misfit dislocations at the interface.

- Since InN likes to be phase separated from GaN, LEDs based on InN/GaN MQWs are expected to be stable against post growth processing such as annealing during formation of Ohmic contacts or degradation during operation.

Figure 25 is a high resolution TEM image of an InN / GaN MQW. As can be seen in these data the $\mathrm{InN}$ is a single monolayer and there is no evidence of misfit dislocations.

Figure 26 shows the schematic of $5 \mathrm{InN} / \mathrm{GaN}$ MQWs grown by MBE on a (0001) GaN template, as well as the photoluminescence spectra of this structure. The peak of the PL spectra is at $380 \mathrm{~nm}$ and the FWHM is $15 \mathrm{~nm}$. These are extremely promising results, since this is approximately the correct wavelength to excite a tricolor phosphor.

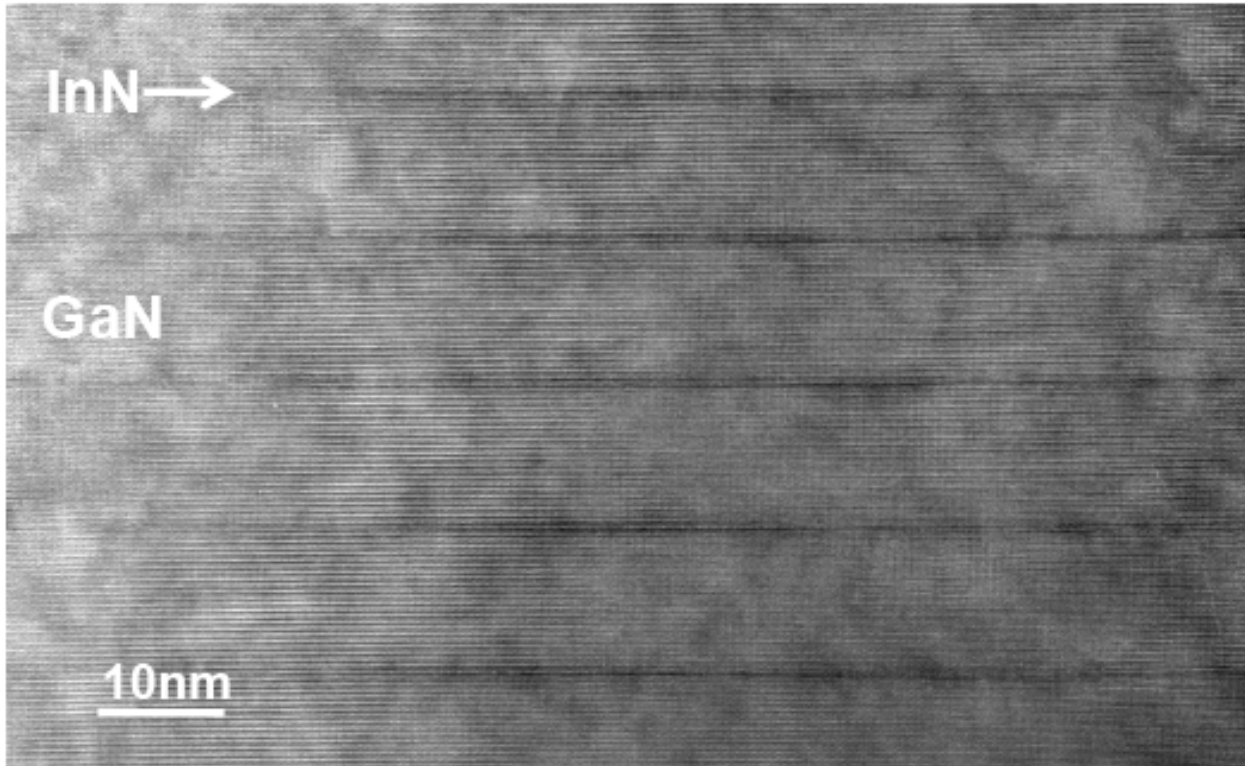

Figure 25: High resolution TEM image of monolayer InN / GaN MQWs 

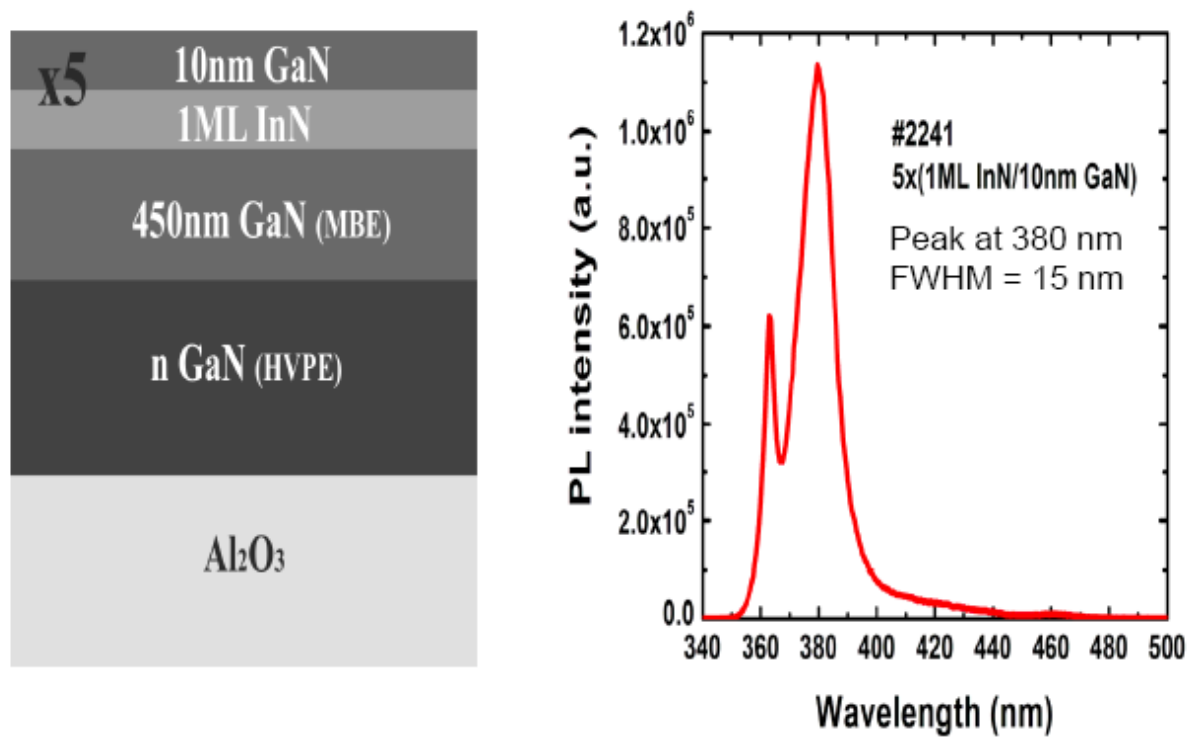

Figure 26: Schematic of 5 monolayer InN / GaN MQWs grown on HVPE GaN template and the PL spectra of this structure.

\section{InGaN/GaN MQWs emitting in the wavelength range 400-550nm}

To study InGaN / GaN MQWs we had to first investigate InGaN thick films grown by MBE on GaN templates. These results were published in a number of student theses and are not going to be repeated in this report $[15,16]$ Instead we will focus on our studies of InGaN/GaN MQWs. However it is important to stress that TEM microscopy in such films reveals the existence of microscopic domains which show atomic ordering. This phenomenon is undesirable in the performance of LEDs.

InGaN/GaN MQWs were grown under a wide range of conditions and evaluated by several methods which were then correlated to the growth conditions. In the majority of cases, growths were carried out on Ga-polar templates on c-plane sapphire. A few samples were also grown on non-polar a-GaN templates, which were deposited by HVPE on the R-plane of sapphire. Changes to the growth conditions included the growth temperature, the flux ratios, ntype doping of the barriers, growth interruption times and film growth duration. Some of these growth conditions were found to play a critical role in the emission behavior of the wells while some were found to have little or no effect. QW samples were then characterized by x-ray diffraction, time and temperature-dependent PL and microscopy.

Additionally, QWs were grown on templates with a modified surface to investigate the role of threading dislocations as non-radiative recombination centers. The emission from these samples was evaluated by temperature and time dependent photoluminescence. Finally, InGaN MQWs grown on a-plane GaN templates are not expected to suffer from the quantum confined 
Stark effect (QCSE) since the polarization fields are in the plane of the QWs rather than perpendicular to the QWs.

\section{C.1: the role of dislocations as non-radiative recombination Centers in InGaN MQWs}

InGaN Quantum Wells (QWs) are used as the active region of blue-green LEDs. These materials and device structures are generally grown hetero-epitaxially on c-plane sapphire or $\mathrm{SiC}$ substrates. Due to large lattice and thermal mismatches between substrate and films, these materials have a high concentration of threading defects (edge, screw and mixed dislocations, and inversion domain boundaries) as well as planar defects (stacking faults) and point defects Furthermore, InGaN alloys tend to undergo both phase separation as well as long-range atomic ordering which give rise to potential fluctuations due to compositional inhomogeneities.

However, in spite of the high concentration of defects in the InGaN alloys, the IQE of multiple QWs and LEDs based on such MQWs was found to be very high [17]. A number of recent papers have addressed the origin of the high internal quantum efficiency in such QWs. For example, Chichibu and co-workers presented arguments that the high IQE is related to localization of excitons in indium-rich regions due to the compositional inhomogeneities [17] Hiegleiter and co-workers presented an alternative model to account for why the threading dislocations are not efficient non-radiative recombination centers [18] These authors have correlated the existence of $\mathrm{V}$-defects in MOCVD-grown InGaN QWs with recombination processes of injected carriers. These V-defects are hexagonal pits with (10-11) sidewalls terminating threading dislocations. The formation of these defects is attributed to the lower growth rate of the (10-11) facets. Specifically, Heigleter et al. have observed that the QWs at the (10-11) facets are thinner than the QWs on (0001) flat areas of the wafer and proposed that electron-hole pairs generated in the flat regions of the MQWs are prevented from recombining at dislocations due to energy barriers between the two types of QWs.

In this paper, we have tested the model proposed by Hiegleiter by growing InGaN MQWs on c-GaN templates which have been intentionally etched to reveal pits associated with screw dislocations. A GaN template, 5 microns thick, was deposited by the hydride vapor phase epitaxy (HVPE) method on a 2 inch (0001) sapphire substrate using the following procedure. First, the sapphire substrate is exposed to $\mathrm{GaCl}$ vapor at $1000^{\circ} \mathrm{C}$ for 30 minutes. We believe that this pretreatment rids the substrate of physisorbed oxygen by forming volatile gallium oxide species and also $\mathrm{Cl}$ reacts with surface carbon to form volatile chlorocarbons. Both of these impurities are undesirable for epitaxial growth. The substrate was then cooled and a lowtemperature $\mathrm{GaN}$ buffer layer, approximately $20 \mathrm{~nm}$ thick, was deposited at $630^{\circ} \mathrm{C}$. The substrate was then ramped up to a temperature of $1020^{\circ} \mathrm{C}$ for the growth of the GaN template.

The template was then quartered and three of the quarters were etched in $\mathrm{HCl}$ gas flow in the same $\mathrm{HVPE}$ reactor at $650^{\circ} \mathrm{C}$ at varying times and $\mathrm{HCl}$ flow rates. One quarter, sample $\mathrm{A}$, was left un-etched; sample B was etched for 25 minutes at $5 \mathrm{sccm}$ of $\mathrm{HCl}$; sample $\mathrm{C}$ was etched for 40 minutes at $5 \mathrm{sccm}$ of $\mathrm{HCl}$; and sample D was etched for 30 minutes at $10 \mathrm{sccm}$ of $\mathrm{HCl}$.

The quarters were then loaded into a MBE system for epitaxial growth of $\mathrm{InGaN} / \mathrm{GaN}$ MQWs. The GaN templates were first exposed to a Gallium flux at $820^{\circ} \mathrm{C}$ a number of times. This process was found to improve the RHEED pattern suggesting that it removes physisorbed oxygen. This was followed by $100 \mathrm{~nm} \mathrm{GaN}$ growth under Ga-rich conditions to establish the 
epitaxial growth. The substrate temperature was then lowered to $670^{\circ} \mathrm{C}$ to grow 7 periods of InGaN/GaN QWs. The wells were $30 \AA$ thick $\operatorname{In}_{.25} \mathrm{Ga}_{.75} \mathrm{~N}$ and the barriers were $35 \AA$ thick GaN. All four samples were grown under identical conditions.

The templates and MQWs were then characterized by scanning electron microscopy (SEM) and Photoluminescence (PL) using a $10 \mathrm{~mW}$ HeCd laser as the excitation source. The PL spectra were also measured as a function of temperature from $10^{\circ} \mathrm{K}$ to $300^{\circ} \mathrm{K}$. The internal quantum efficiency (IQE) at room temperature of the InGaN MQWs was calculated by taking the ratio of the integrated PL intensity at room temperature divided by the integrated PL intensity at $10^{\circ} \mathrm{K}$. The determination of the IQE by this method assumes that the excitons at $10^{\circ} \mathrm{K}$ are localized and thus all recombination processes are radiative.

Figure 27 shows the SEM surface morphology of sample B after etching. Etching with $\mathrm{HCl}$ under these conditions, reveals a network of etch-pits associated with screw threading dislocations [19]. In this reference, the authors have conducted detailed electron microscopy and atomic force microscopy (AFM) studies, and concluded that the screw dislocations are decorated with (10-12) hexagonal pits. We have examined our etched $\mathrm{GaN}$ films by AFM and found that the angle of the facets was approximately $45^{\circ}$ off of the $(0001)$ planes. Thus, these facets correspond to the (10-12) planes which are $43.2^{\circ}$ off the c-plane. It should be noted that the planes of these pits are different than the planes of the pits formed during the growth of InGaN which, as mentioned earlier, are (10-11). The density of the pits from the data of Figure 27 was found to be $\sim 4 \times 10^{8} \mathrm{~cm}^{-2}$. For samples $C$ and $D$, which were etched more extensively, we found that the density of the pits is the same, however, their size increases with the degree of etching until they begin to coalesce. Thus, the area of the template's c-plane surface becomes progressively smaller as the etching proceeds. Figure 28 shows the SEM surface morphology of all four samples upon completion of the InGaN MQW growth. As seen from this data, the MQWs have replicated the GaN template.

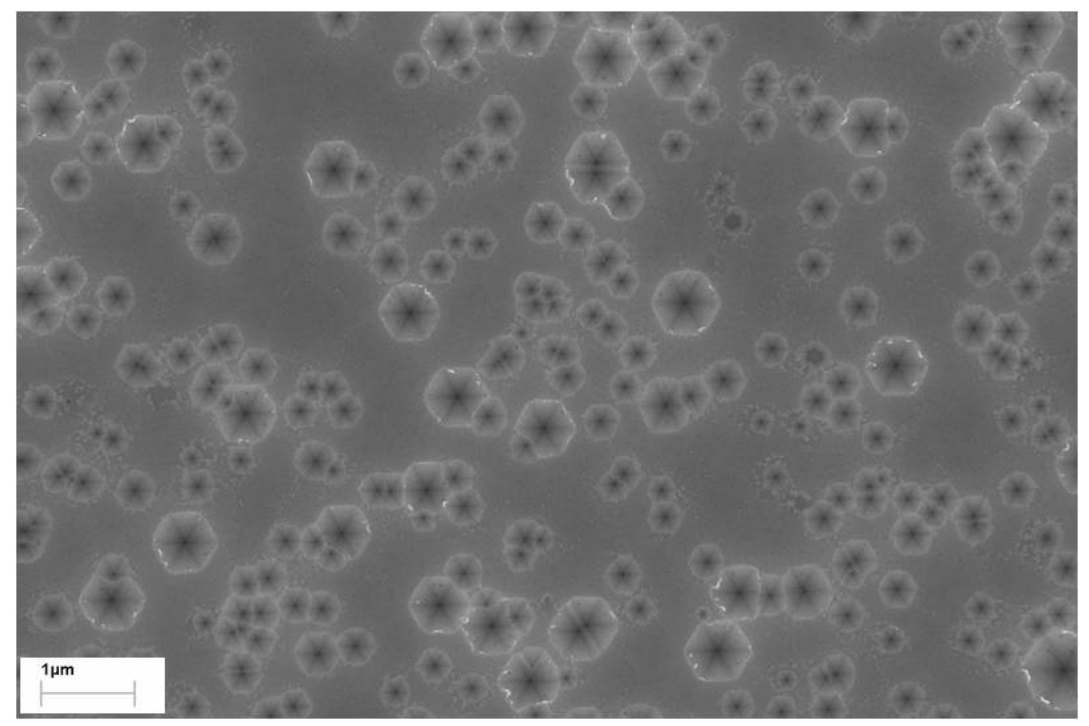

Figure 27: SEM surface morphology of an HVPE-grown GaN template after etching at $600^{\circ} \mathrm{C}$ for 25 minutes in $5 \mathrm{sccm}$ of $\mathrm{HCl}$ 


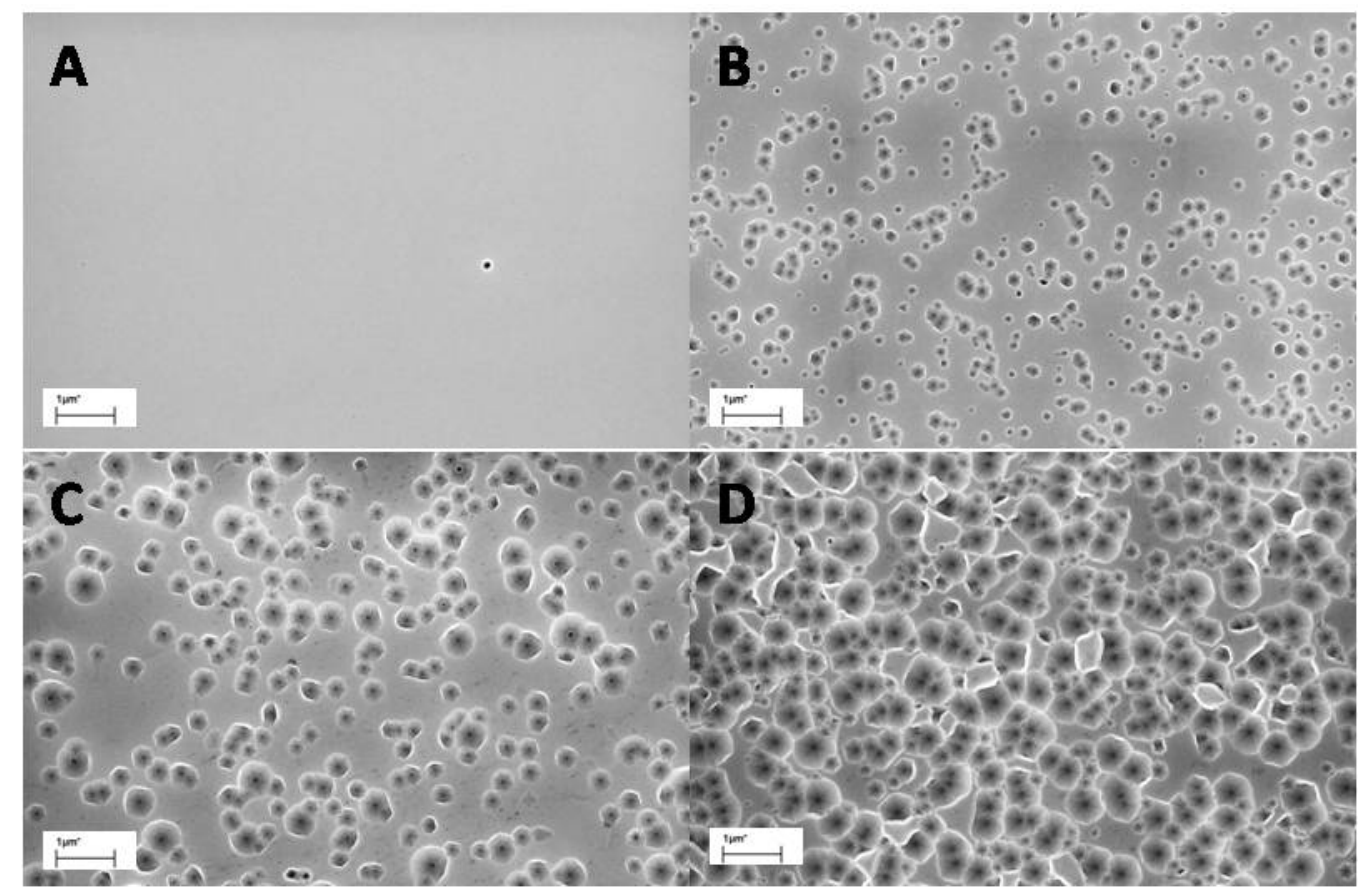

Figure 28: SEM surface morphology of samples A, B, C and D upon completion of the InGaN MQW growth.

Figure 29 shows the room temperature PL spectra of each sample. All four spectra have a main peak in the spectral region between 480 to $490 \mathrm{~nm}$ with FWHM approximately $50 \mathrm{~nm}$. The etched samples have an additional peak in the spectral range of 420 to $430 \mathrm{~nm}$ with FWHM approximately $22 \mathrm{~nm}$. The intensity of this second peak increases with the degree of etching of the GaN template prior to the growth of the QWs. This result suggests that the origin of this peak is related to QWs on the inclined (10-12) planes. Additionally, the existence of interference fringes in the main peak in the PL spectra of all samples is evidence that these spectra are due to emission from the (0001) planar MQWs. On the other hand such interference fringes are absent from the second peak at shorter wavelengths indicating that the origin of this peak is due to emission from the inclined (10-12) MQWs. 


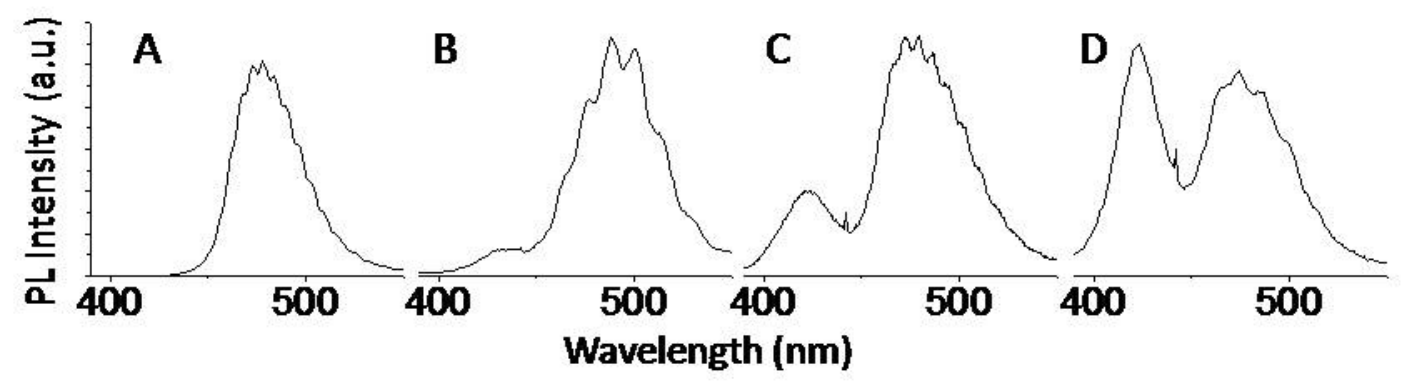

Figure 29: The room temperature PL spectra for samples A, B, C and D.

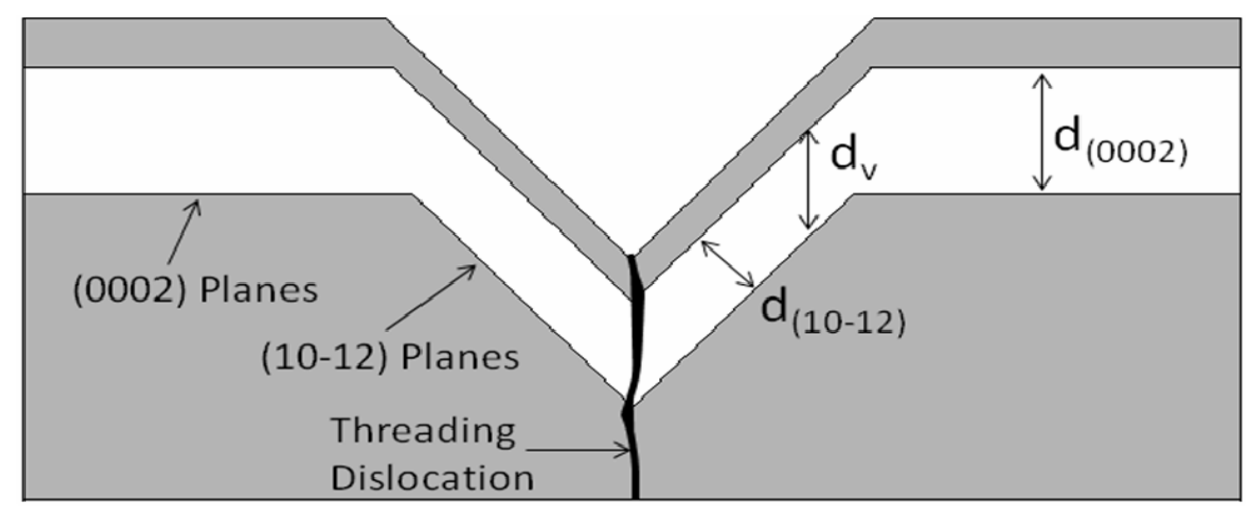

Figure 30: Schematic of the area around the (10-12) pit after the QW growth. All relevant well thicknesses and growth planes are labeled.

To quantitatively account for the PL spectra of the MQWs, we show in Figure 30 a schematic of the area around the (10-12) pit after the QW growth. From electron microscopy studies reported in Figure 18, the MQWs on the (10-12) facets are thinner than those on the (0001) planes. There are two factors which account for this observation. First, the arriving fluxes of the $\mathrm{Ga}$, In and $\mathrm{N}$ beams are distributed over a larger area in the pits than the corresponding c-plane surface. Thus we expect the thicknesses of the InGaN wells measured in the c-direction on the (10-12) planes to be $d_{v}=d_{(0001)} \times \cos (43.2)$, where $d_{(0001)}$ is the thickness of the (0001) plane QW. Second, by taking into account the geometrical shape of the pits, the thickness of the (10-12) wells in the confinement direction is $\mathrm{d}_{(10-12)}=\mathrm{d}_{\mathrm{v}} \mathrm{x} \cos (43.2)$. Therefore, the thickness of the (10-12) wells is expected to be 0.53 times the thickness of the wells on the cplane. Because the QWs are thinner in the inclined (10-12) facets, emission from those QWs is expected to be blue-shifted with respect to the (0001) QWs. Furthermore, the polarization vectors in the (10-12) QWs are no longer perpendicular to the well and so the piezoelectric fields are similarly reduced. This lessens the quantum-confined-Stark-effect (QCSE) which leads to an even larger blue-shift from the wells grown on the (0001) planes. Taking into account both the thicknesses of the QWs as well as the polarization effects, we calculated the emission energies by solving the Poisson and Schrodinger equations independently. The emission for these MQWs 
is $439 \mathrm{~nm}$ and $492 \mathrm{~nm}$ for the (10-12) and (0001) wells respectively, in approximate agreement with the observed PL spectra.

The IQE of the main peak in the PL spectra was determined by measuring the PL spectra as a function of temperature for all four samples. Figure 31 shows an Arrhenius plot of the integrated PL intensity I(T) for sample A.

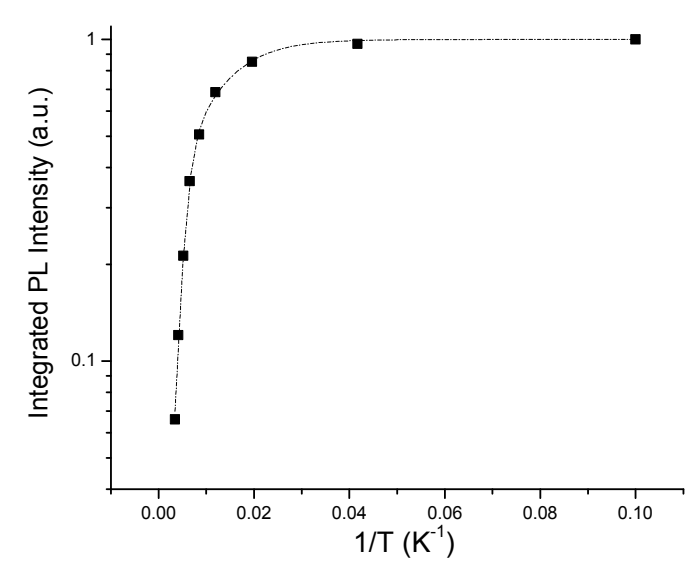

Figure 31: Arrhenius plot of the integrated PL intensity, I(T), for sample A.

The data of Figure 31 were fit using the following expression:

$$
I(T)=\frac{I(0)}{1+A_{1} \exp \left(-\frac{E_{A 1}}{k_{B} T}\right)+A_{2} \exp \left(-\frac{E_{A 2}}{k_{B} T}\right)}
$$

The two activation energies for non-radiative recombination processes, $\mathrm{E}_{\mathrm{A} 1}$ and $\mathrm{E}_{\mathrm{A} 2}$, were found to be $13 \mathrm{meV}$ and $79 \mathrm{meV}$ respectively and correspond to two different mechanisms of PL quenching. The low temperature quenching mechanism of $13 \mathrm{meV}$ is attributed to thermal delocalization of excitons from potential fluctuations arising from thickness variations in the wells or compositional inhomogeneities. The high temperature quenching mechanism of 79 $\mathrm{meV}$ is attributed to the lateral diffusion of carriers to dislocations where they recombine nonradiatively. Similar analysis was carried out for the etched samples B, C and D and we found that the low temperature quenching mechanism is approximately the same as sample A while the high temperature quenching has increased for the etched samples to approximately $95 \mathrm{meV}$. This result suggests that there is a larger barrier for non-radiative processes for the etched samples.

Figure 32 shows the room temperature IQE of the main peak for all four samples. The IQE of sample A is $4.9 \%$ while the IQE for the etched samples is approximately $10 \%$. This result is also consistent with the calculated barrier for non-radiative processes discussed previously. This conclusion qualitatively agrees with the model proposed by Heigleiter and co- 
workers that the carriers from the (0001) MQWs have to overcome an energy barrier in order to migrate to dislocations where they can recombine non-radiatively.

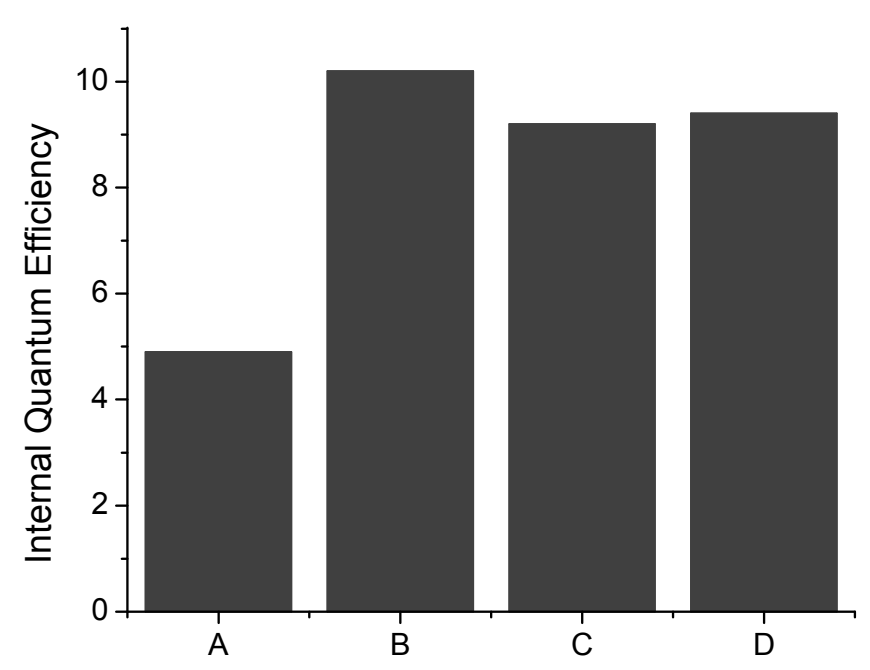

Figure 32: Room temperature IQE of the main peak at $\sim 485 \mathrm{~nm}$ for samples $\mathrm{A}, \mathrm{B}, \mathrm{C}, \mathrm{D}$

In conclusion, we have demonstrated the importance of suppressing non-radiative recombination in screw dislocations in InGaN MQWs by growing such structures on GaN templates which have previously been etched to reveal (10-12) pits associated with screw dislocations. Specifically, we found that the IQE of the MQWs grown on the etched c-GaN template is a factor of 2 higher than similar QWs grown on atomically smooth surfaces. This result was accounted for by the fact that the QWs on the (10-12) planes are significantly thinner than the c-plane QWs in agreement with the model proposed recently by Hangleiter et al.

\section{C.2: the effect of growth temperature}

Another growth parameter that affected the efficiency of InGaN MQWs was the growth temperature. Figure 33 shows the internal quantum efficiency for several samples. Although, they were grown on a variety of different substrates under varying conditions, a trend is visible with respect to temperature. The IQE increases with temperature and has a peak at a growth temperature of $690^{\circ} \mathrm{C}$. Above $700^{\circ} \mathrm{C}$ the efficiency quickly falls off. This is not surprising given that the growth of InGaN above $700^{\circ} \mathrm{C}$ is difficult and indium in the well will quickly diffuse out during the growth interrupt. The increases in efficiency with growth temperature, for temperatures below $690^{\circ} \mathrm{C}$ is attributed to increasing disorder in the wells leading to better carrier confinement. 


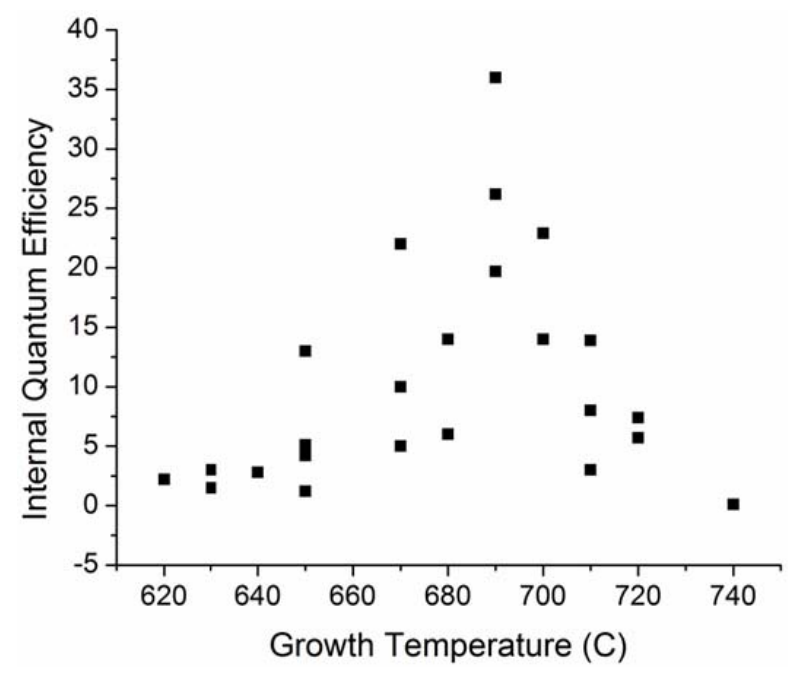

Figure 33: IQE vs. growth temperature for several InGaN MQW samples

\section{Task 1.4 Epitaxial Growth of UV / Blue / Green LEDs by MBE and MOCVD on Textured GaN Templates}

In this subtask we have evaluated the growth and characterization of LEDs by probing at the wafer. Figure 34 shows a schematic of the LED structures investigated and the corresponding band structure of the device under forward bias. In the previous subtasks we had discussed the growth and optimization of the various layers of the LED device.

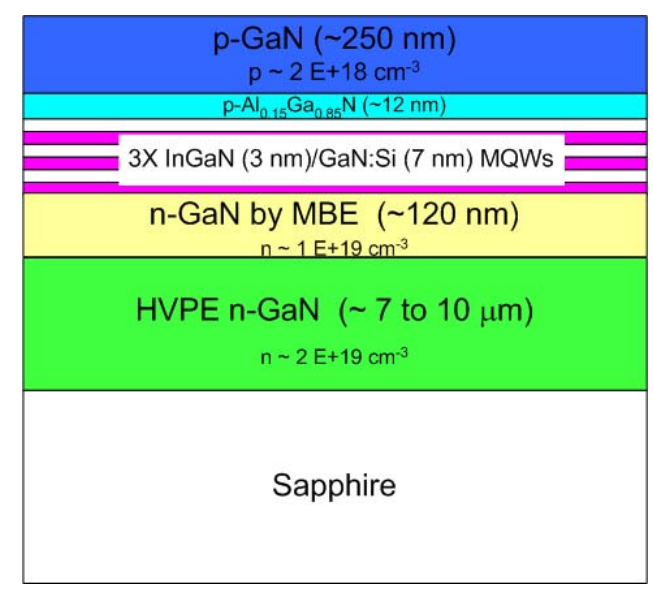

Schematic of an LED structure

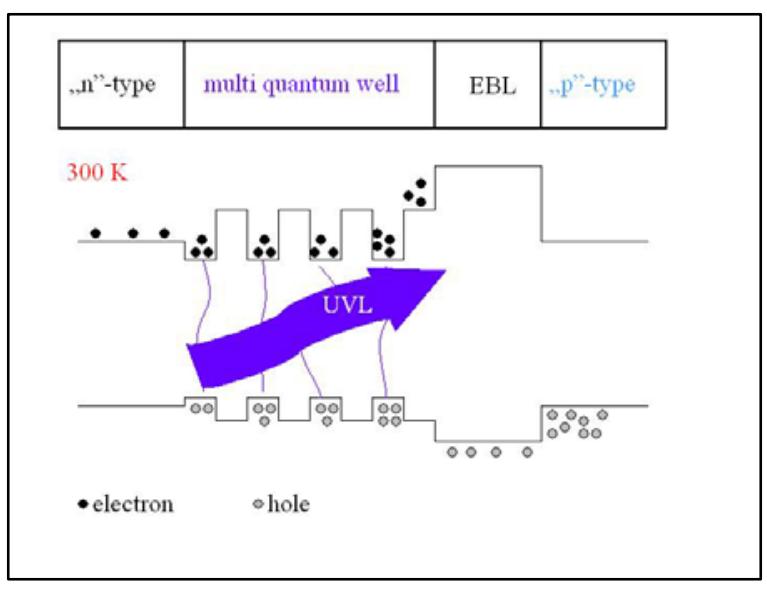

Band structure of an LED structure

Figure 34: Schematic of the investigated LED devices and the corresponding band structure under forward bias. 
After growth each LED wafer was evaluated by probing as indicated in Figure 35. Specifically, the wafer was scratched with a scriber in one corner in order to reveal the bottom n-GaN layer and In was soldered at this point. A tungsten probe was used to contact the bottom n-GaN, while a Gold probe was used to contact the $\mathrm{p}-\mathrm{GaN}$. Gold was used because of its larger work function. The significant illumination emitted by the wafer in this crude test is an indication that the growth was successful and therefore we have proceeded with the various steps of device fabrication. Below we present some examples of probing at the wafer level various LED structures developed in this program.

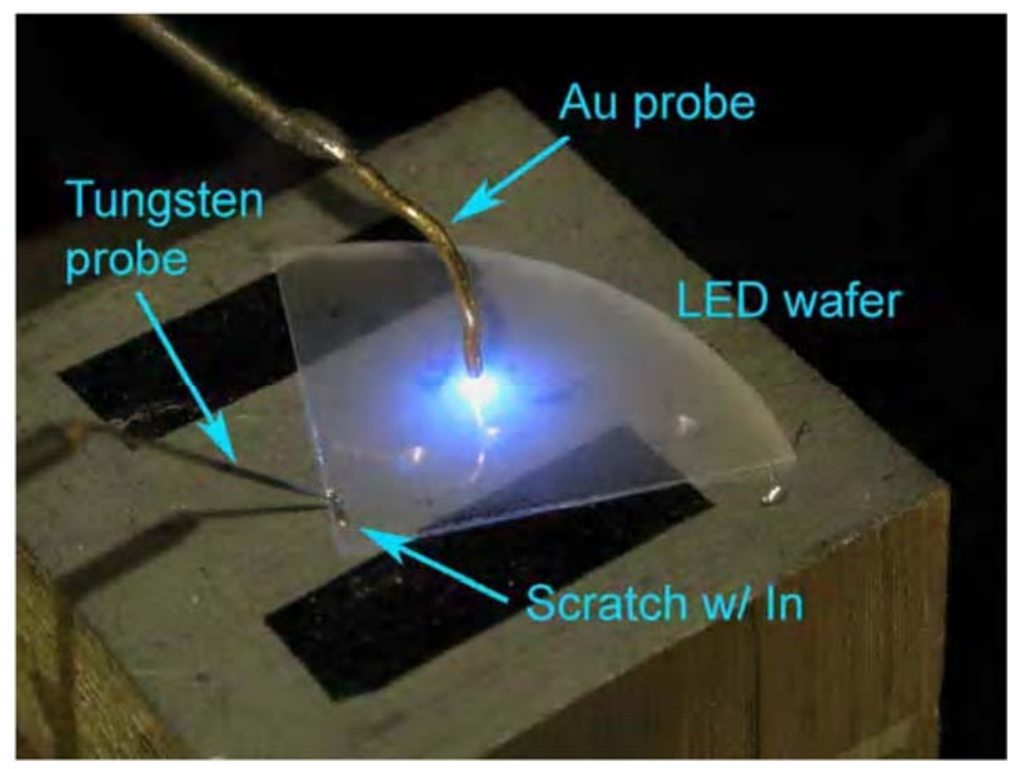

Figure 35: Wafer level probing of an LED structure.

Wafer-level EL spectra from InN/GaN MQW based LED structure is shown in Figure 36. The device was probed at $5 \mathrm{~mA}$ and $20 \mathrm{~mA}$ and emits at $290 \mathrm{~nm}$.
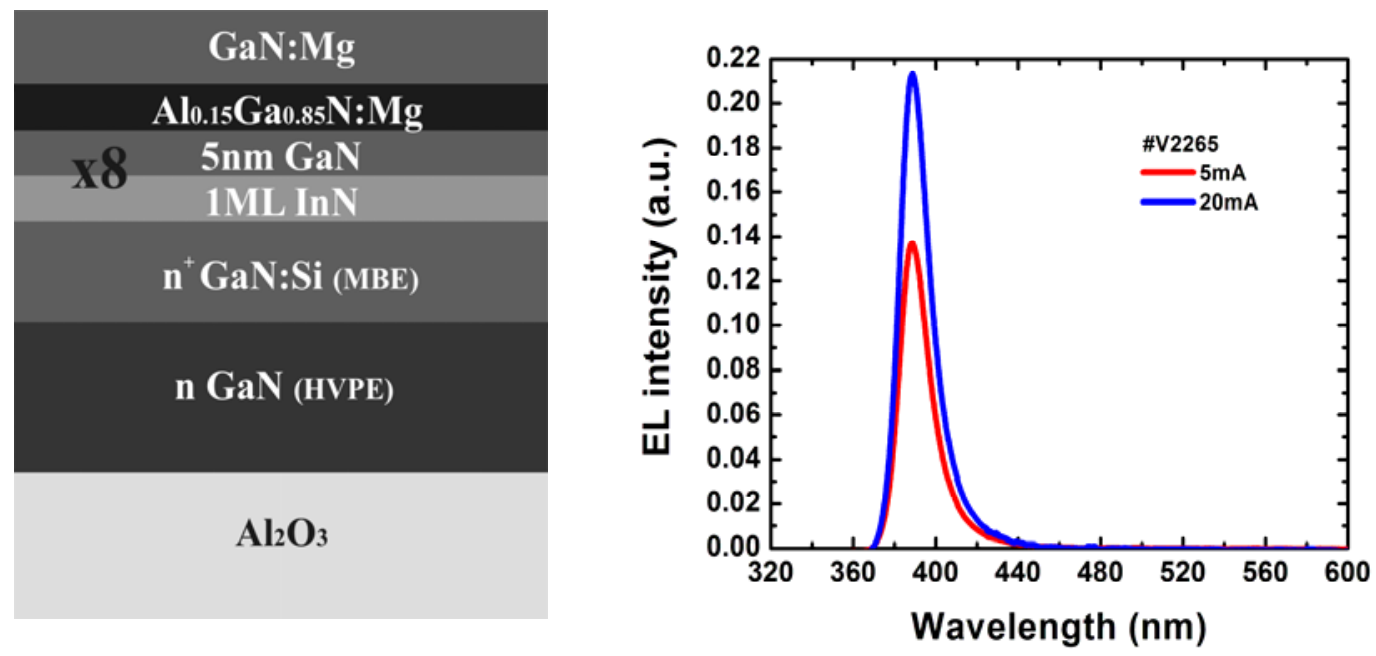

Figure 36: (Left panel) Schematic of an LED structure based on monolayer InN /GaN MQWs; (Right panel) Electroluminescence spectra of this LED structure collected at the wafer 1 
Task 1.5: Fabrication and characterization of LED structures on smooth and textured GaN templates.

\section{A. Device Fabrication Procedures}

The details on the fabrication of the LED structures of this project were published in the Boston University Ph.D. dissertation of Dr. Cabalu [15] In this report we present only a brief summary of the fabrication process.

Figure 37 shows photographs of two processed LED devices with smooth surface morphology. The first is an $800 \mu \mathrm{mX} 800 \mu \mathrm{m}$ mesa, while the second one shows a device with $300 \mu \mathrm{mX} 300$ $\mu \mathrm{m}$ mesa. The other structures in the picture are alignment marks or test structures to measure the contact resistivity etc.

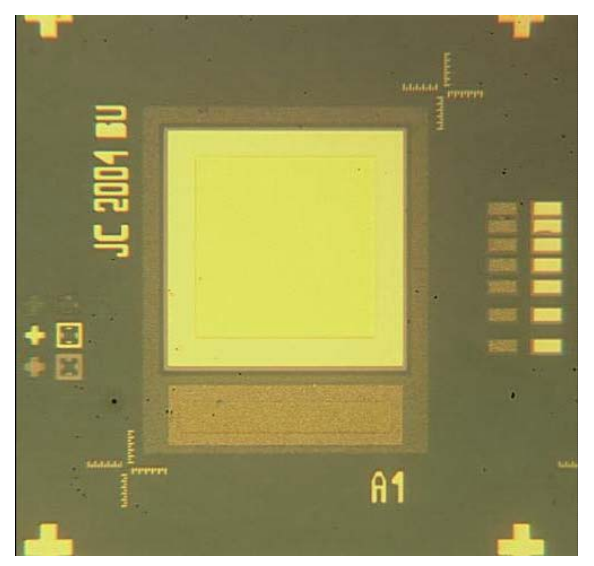

$800 \mu \mathrm{m} X 800 \mu \mathrm{m}$ mesa

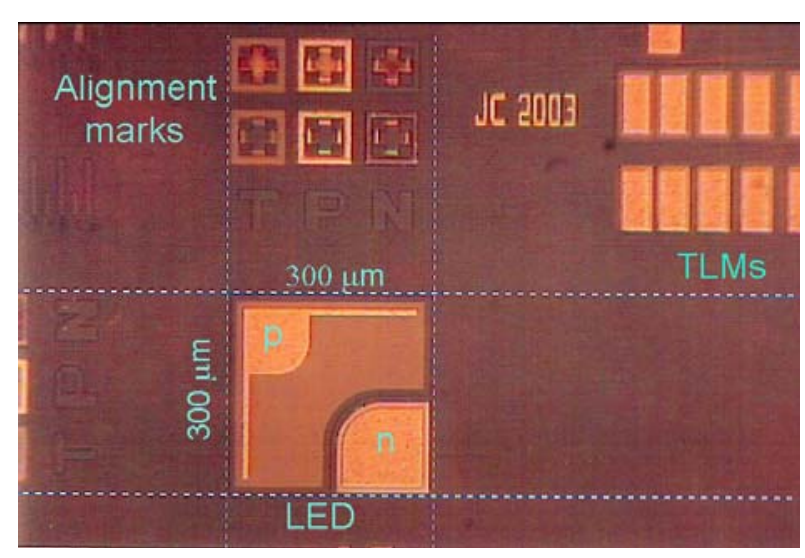

$300 \mu \mathrm{m} X 300 \mu \mathrm{m}$ mesa

Figure 37: Photographs of fabricated KLED devices

The fabrication of LED devices on Textured GaN Templates is much more involved because of the difficulty in viewing the alignment marks during photolithography. A schematic of the fabricated textured LED device is shown in Figure 38 and Figure 39 shows a photograph of a completely fabricated textured LED device. Figure 40 is a photograph of a $1 \mathrm{~cm} \times 1 \mathrm{~cm}$ chip with UV LEDs arrays and test structures and Figure 41 are processed LED devices prior to dicing. 


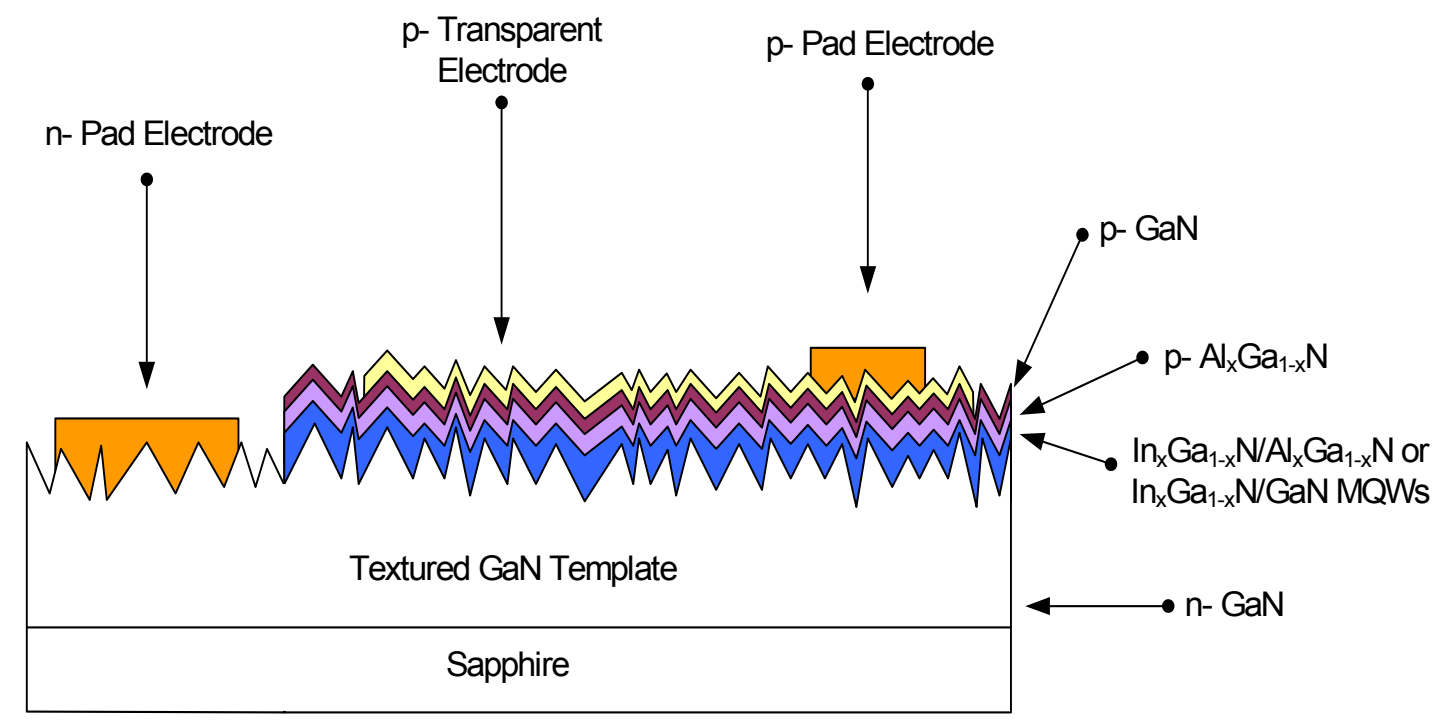

Figure 38: Schematic of fabrication of a textured LED device.

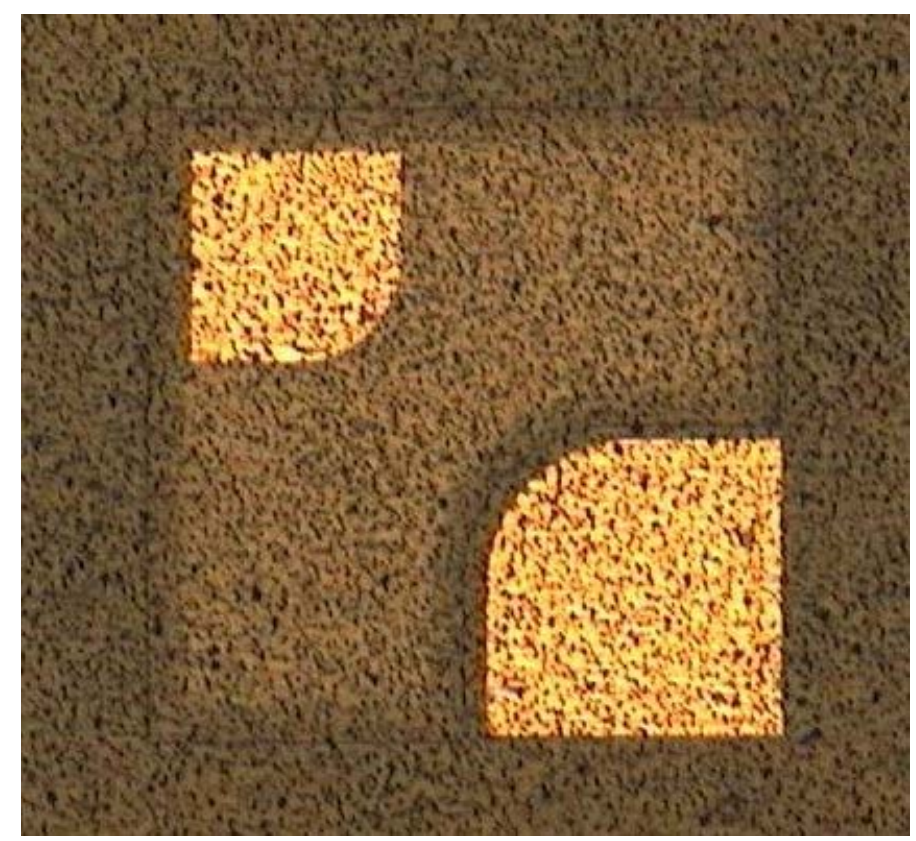

- mesa was formed by chlorine-based ICP etching

- p-ohmic contacts: $\mathrm{Ni}$ / Au

- n-ohmic contacts: Ti / Al / Ni / Au

Figure 39: A photograph of an LED structure employing textured InGaN MQWs. 


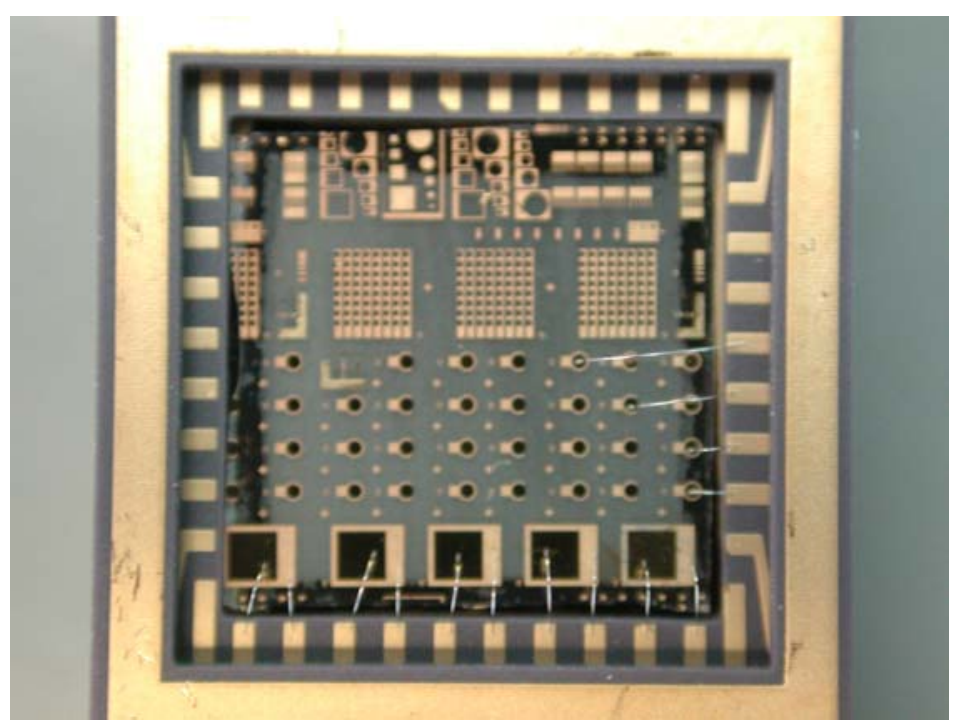

Figure 40: A photograph of a $1 \mathrm{~cm} \times 1 \mathrm{~cm}$ chip with UV LEDs arrays and test structures

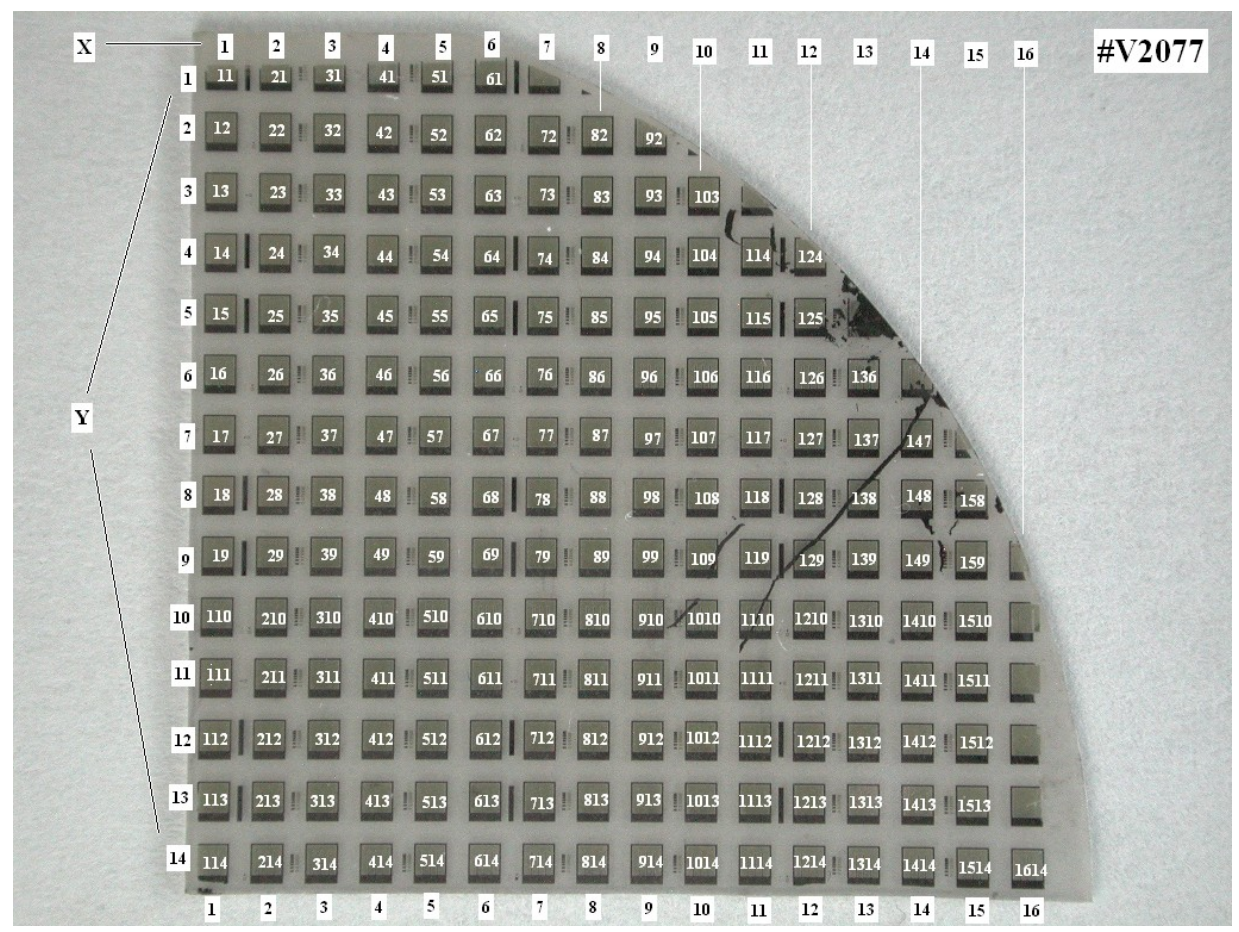

Figure 41: Processed LED devices in a $1 \frac{4}{4}$ of a two inch wafer prior to dicing.

A few devices were also flip-chip bonded on a silicon submount. A schematic of a flip chip bonded device is shown in Figure 42. For the flip-chip bonding process we used either In bumps or Au-stud bumps as shown in Figure 43. 


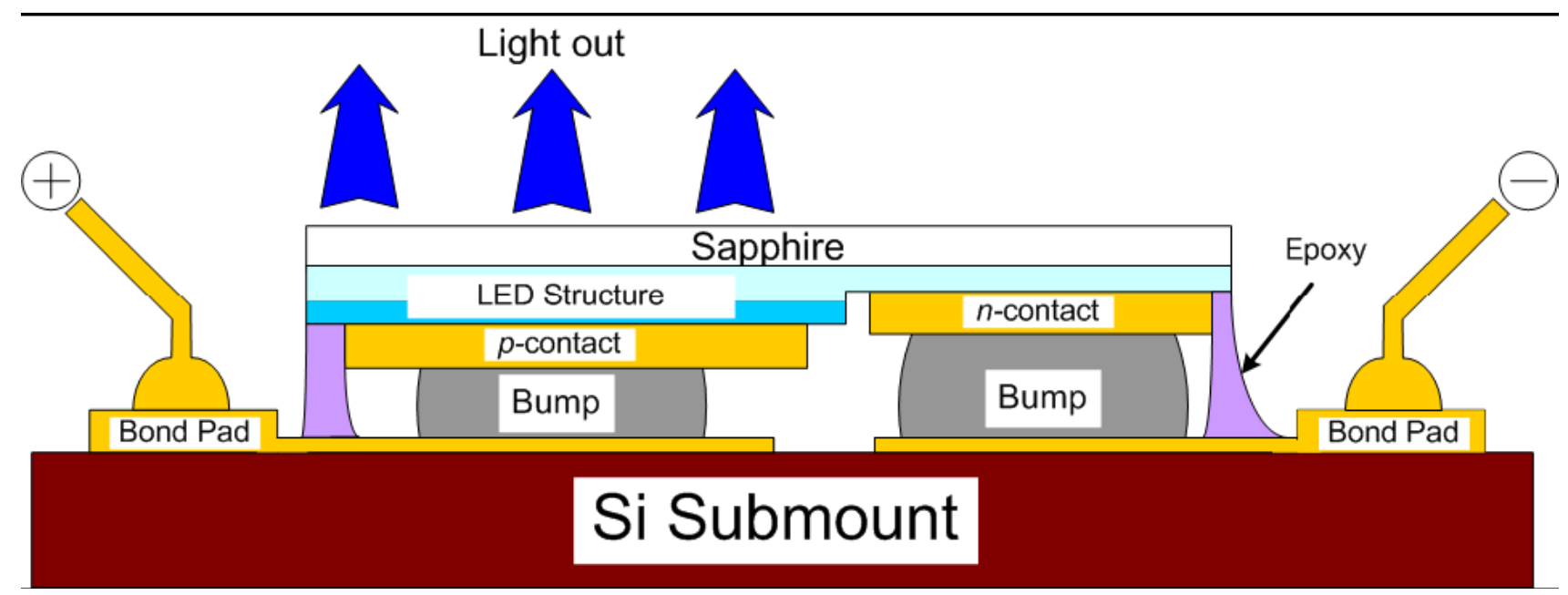

Figure 42: Schematic of a flip-chip-bonded device

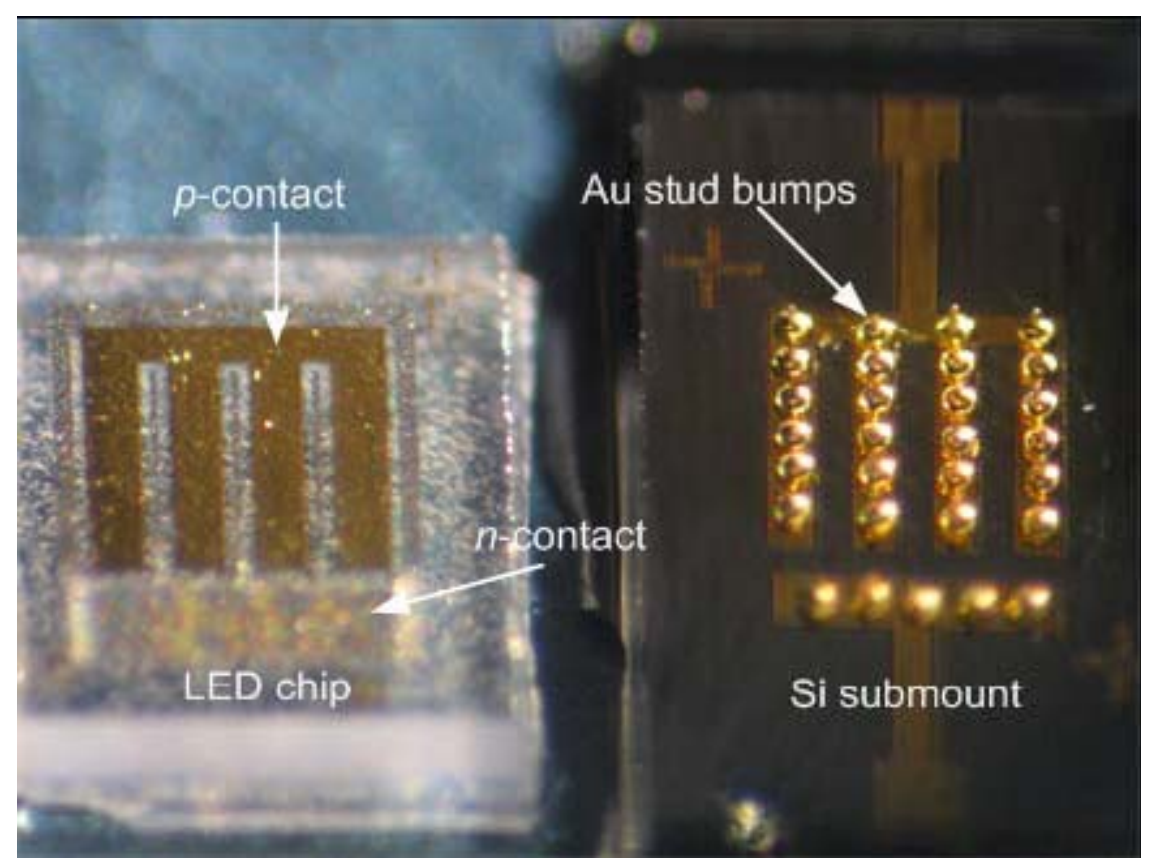

Figure 43: A photograph of a diced LED chip and Si submount ready for flip-chip bonding.

Figure 44 shows a photograph of a semi-packaged LED device. 


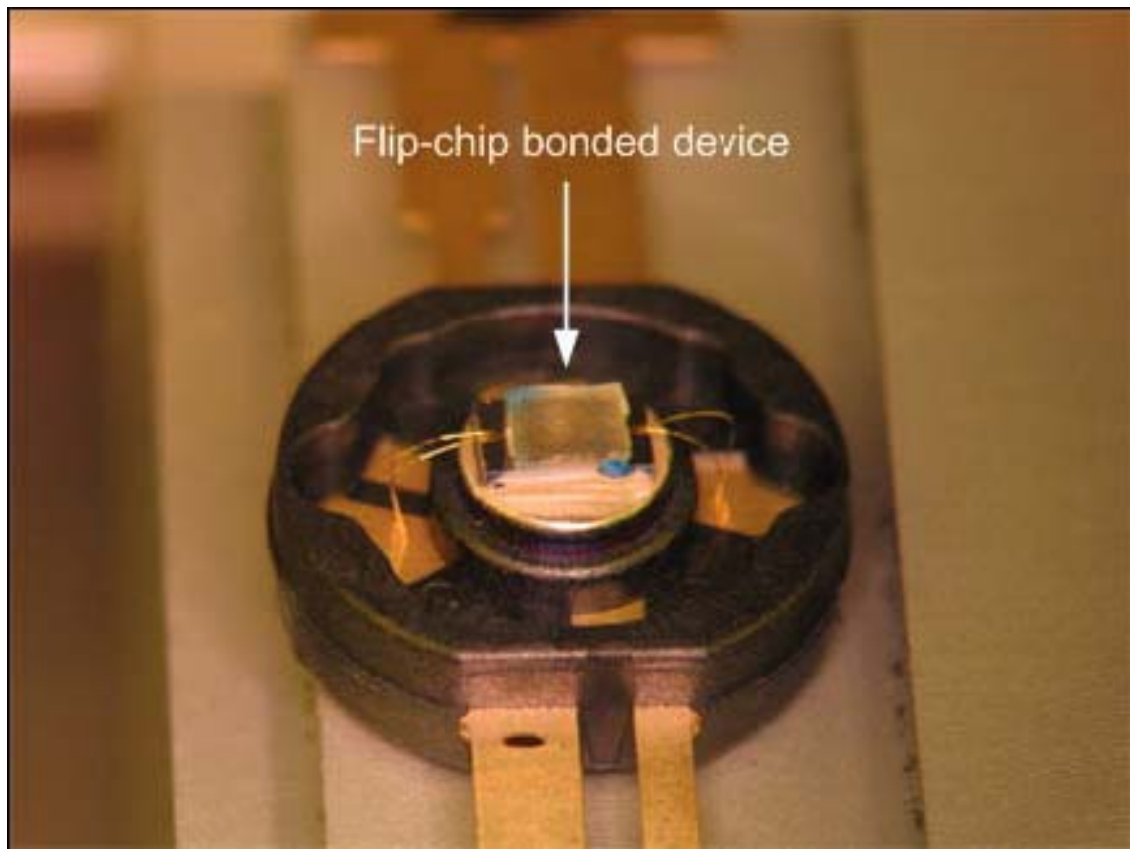

Figure 44: Photograph of a semi-packaged flip-chip bonded LED grown by PAMBE on smooth HVPE GaN template.

\section{B. Device evaluation procedures}

These semi- packaged LED structures tend to heat up during operation by more than $100{ }^{\circ} \mathrm{C}$, which degrades their overall performance. The optical power of the devices was measured at a bare die configuration as indicated in Figure 45. In this configuration the LED device rest on the top of a double polished sapphire wafer and the opening of the integrated sphere is placed under the sapphire wafer. Thus only a very small fraction of light is collected in the integrating sphere. 

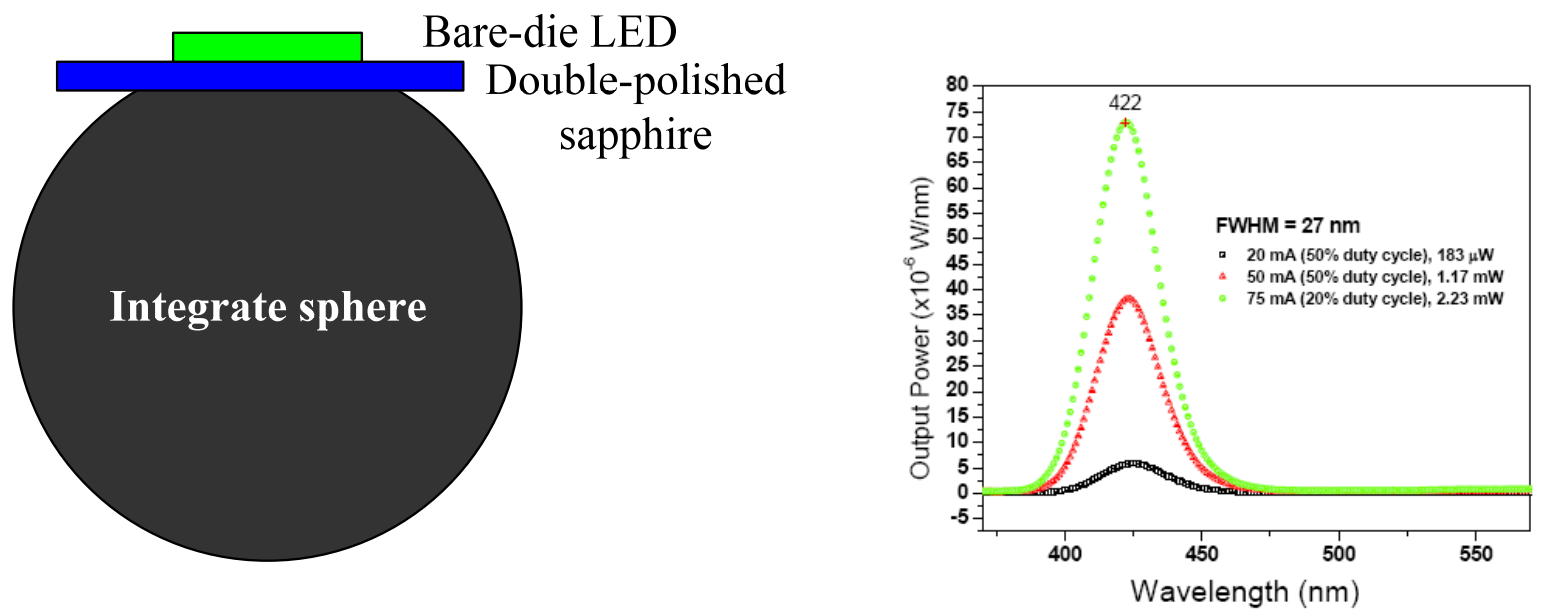

Figure 45: Optical power measurement of bare-die LEDs and an example of EL spectra evaluated using this method

In the following few figures we present the characterization of a few LEDs using the method described in Figure 45. Figure 46 is the schematic of an LED structure and the EL spectra of a device grown on a smooth GaN HVPE template. The device was evaluated up to $1 \mathrm{~A}$ injection current and the peak emission occurs at $450 \mathrm{~nm}$.

\begin{tabular}{|c|}
\hline $0.2 \mu \mathrm{m}$ p-GaN \\
\hline $8 \mathrm{~nm}$ AlGaN \\
\hline $6 \times \mathrm{InGaN} / \mathrm{GaN}$ MQWs $(3 \mathrm{~nm} / 4 \mathrm{~nm})$ \\
\hline $1 \mu \mathrm{m} \mathrm{GaN} \mathrm{template} \mathrm{(MBE)}$ \\
\hline $5 \mu \mathrm{m}$ HVPE GaN template \\
\hline c-plane sapphire \\
\hline
\end{tabular}

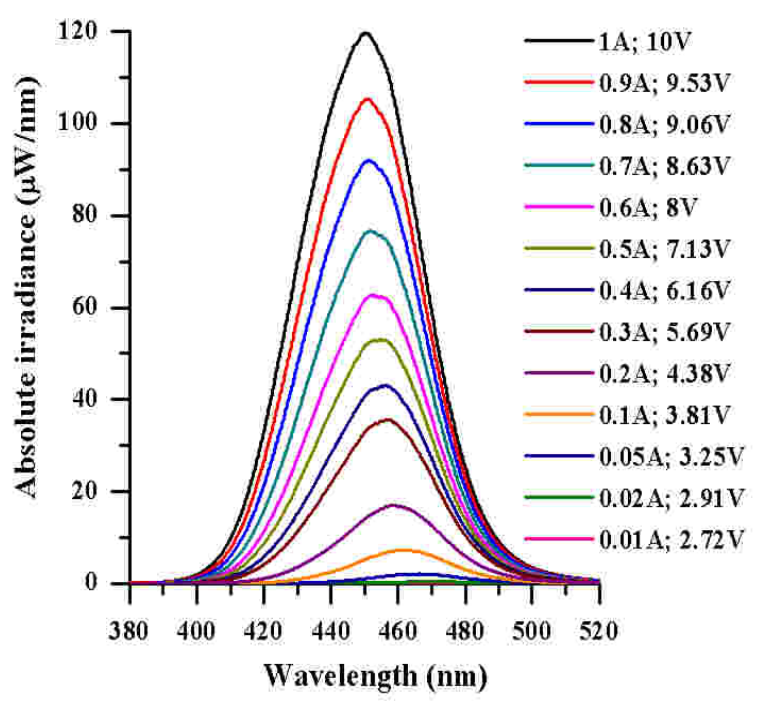

Figure 46: Schematic and EL spectra of an LED structure grown by MBE on a smooth HVPE (0001) GaN template. 
Figure 47 shows the absolute magnitude of the measured optical power from the LED of Figure 46 measured using the method of Figure 45.

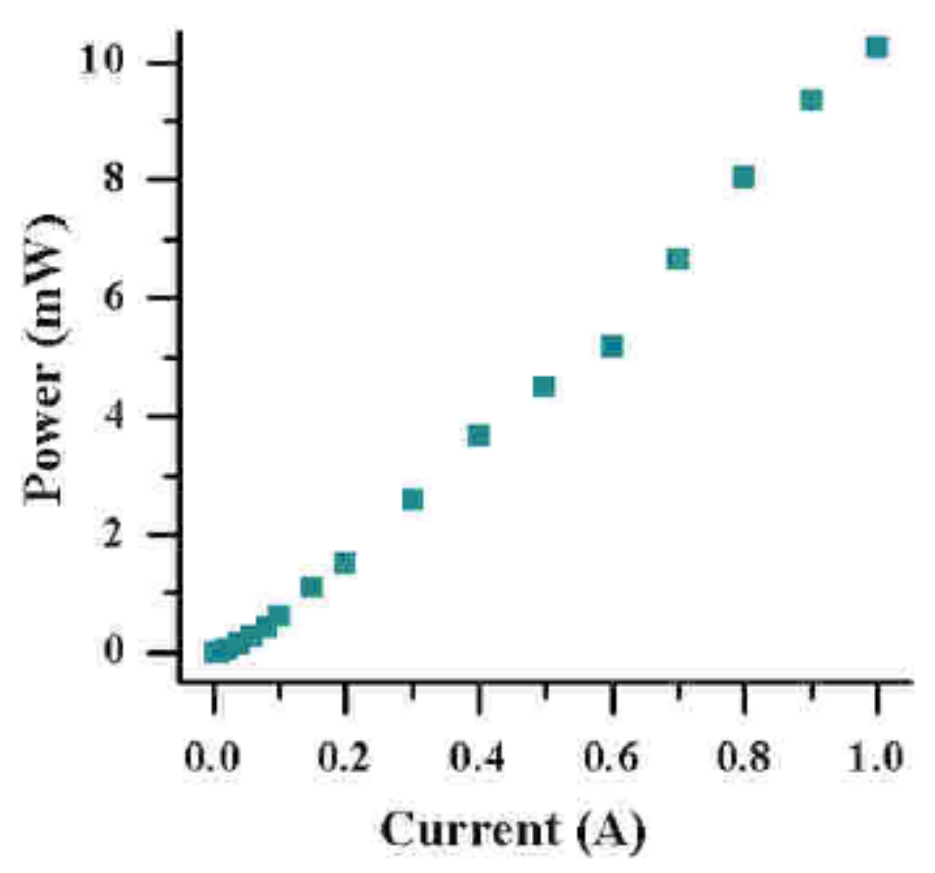

Figure 47: Optical power from the LED of Figure 46 measured using the method of Figure 45.

Figure 48 shows electroluminescence spectra of LEDs whose emission varies from near UV to green grown by PAMBE on Smooth GaN Templates. Figures 49 and 50 show the pictures and the I-V characteristics of the blue and green LEDs. Figure 51 shows photographs of semipackaged blue, turquoise and green LEDs biased to the same injection current. These devices were all grown on smooth HVPE GaN templates. 


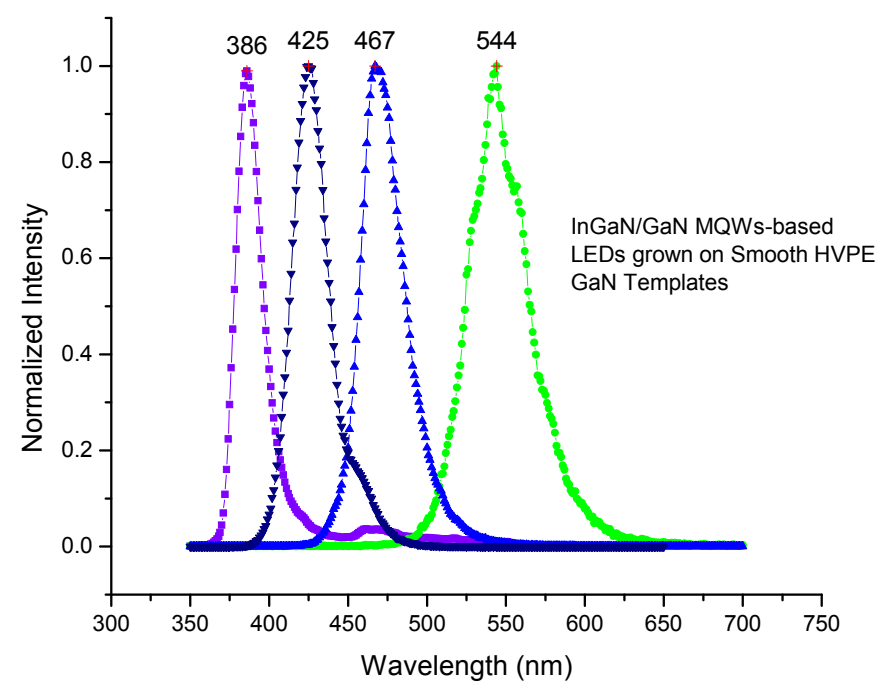

Figure 48: EL spectra of LEDs whose emission varies from near UV to green grown by PAMBE on Smooth GaN Templates
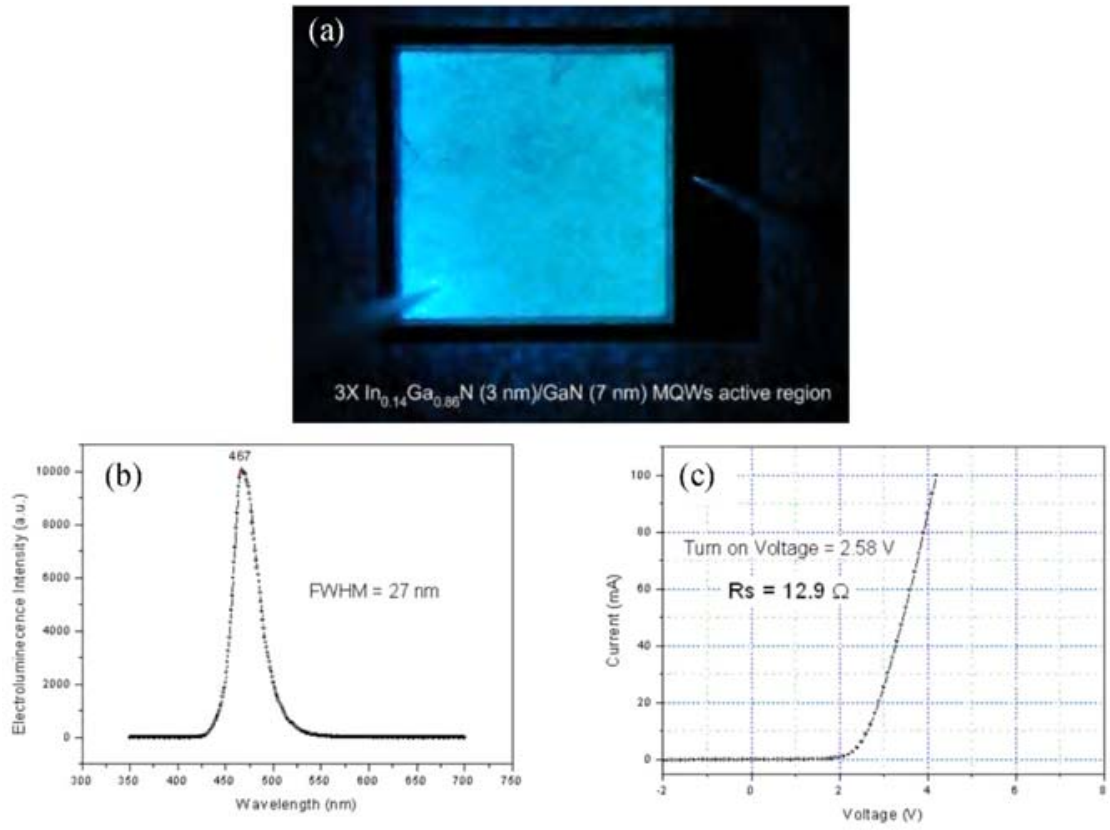

Figure 49: A photograph and I-V characteristics of the blue LED discussed in Figure 47 

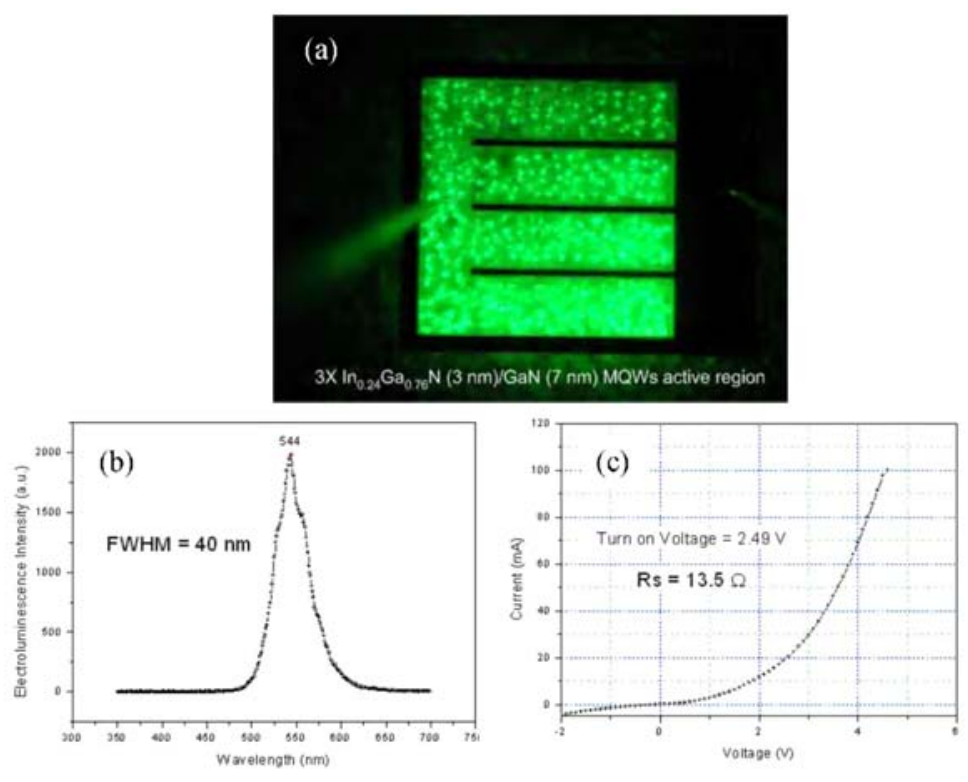

Figure 50: A photograph and I-V characteristics of the green LED discussed in Figure 47.

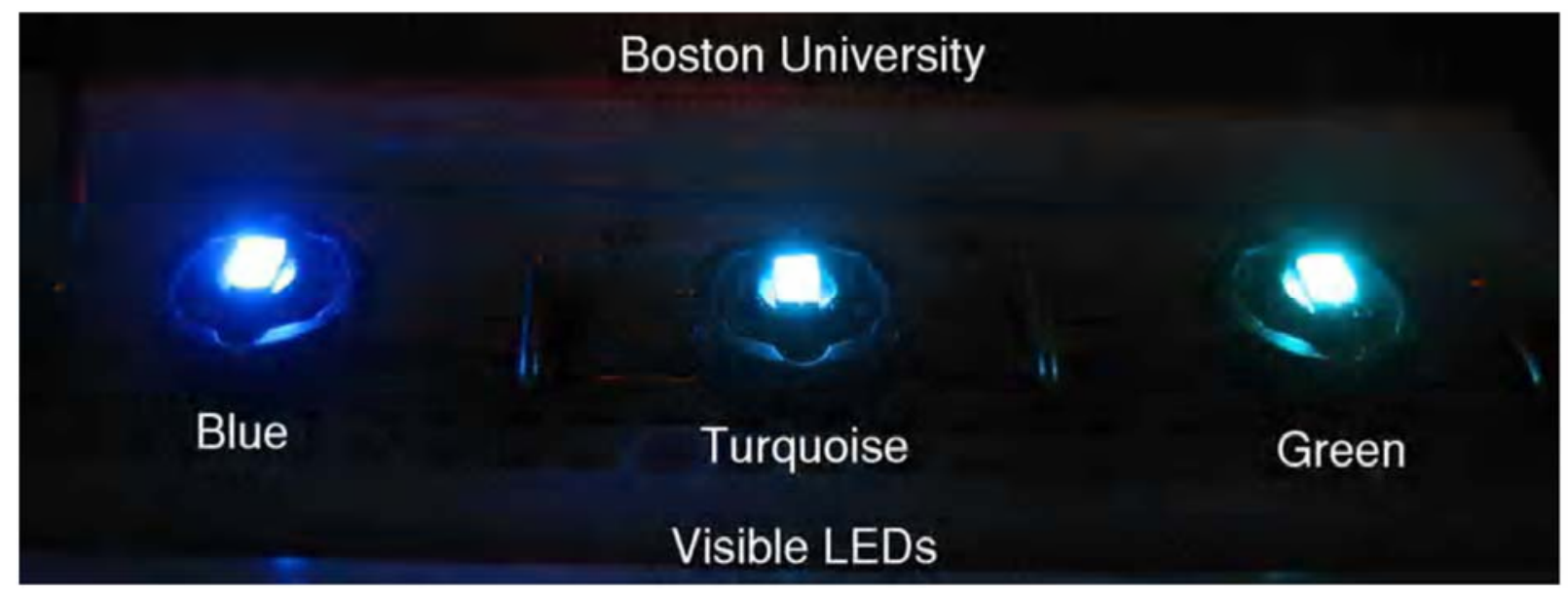

Figure 51: Semi-packaged LEDs of different colors grown by MBE on smooth HVPE GaN templates. All three devices were injected with the same current

\section{Variable color LEDs based on textured InGaN MQWs grown on textured GaN substrates}

As discussed in Figure 29 textured InGaN MQWs emit in multiple wavelengths. This was attributed to narrower thickness of the wells in the inclined planes compared to the (0001) 
planes. The same result was observed in LEDs grown on textured GaN templates. An example is shown in Figure 52. Under low injection current $(20 \mathrm{~mA})$ the LED emits at $340 \mathrm{~nm}$ (green). However as the device is biased at higher voltage the device emits both in the green and blue. Under even higher bias (injection current of $240 \mathrm{~mA}$ ) the mixture of the blue and green produces white color. Thus this method is capable of producing white color without the use of phosphor (phosphorless white LED)
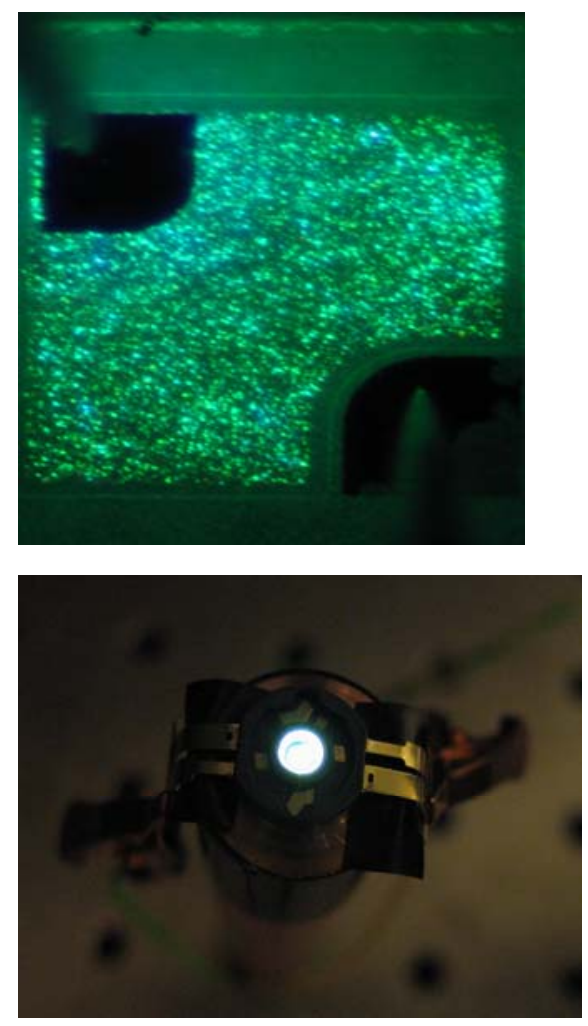

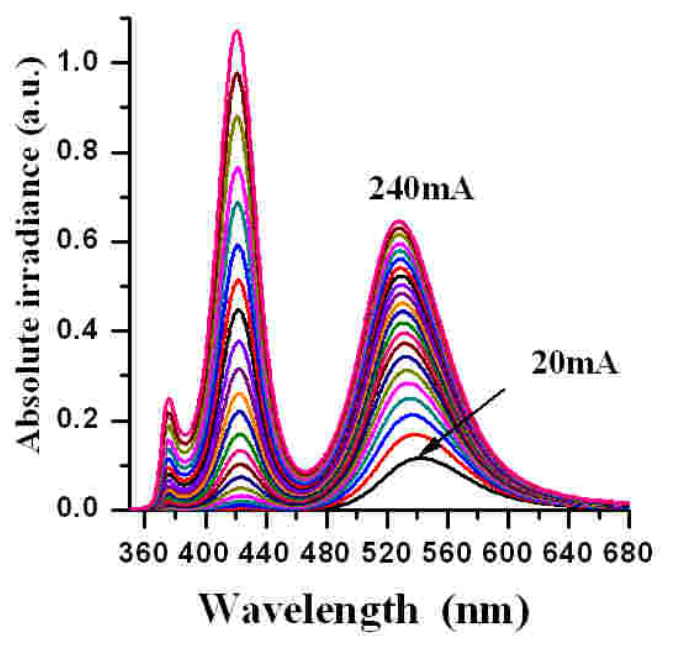

Figure 52: Variable color LED based on textured InGaN MQWS

For comparison we show in Figure 53 the EL spectra of a commercial white LED based on a blue LED and yellow phosphor.

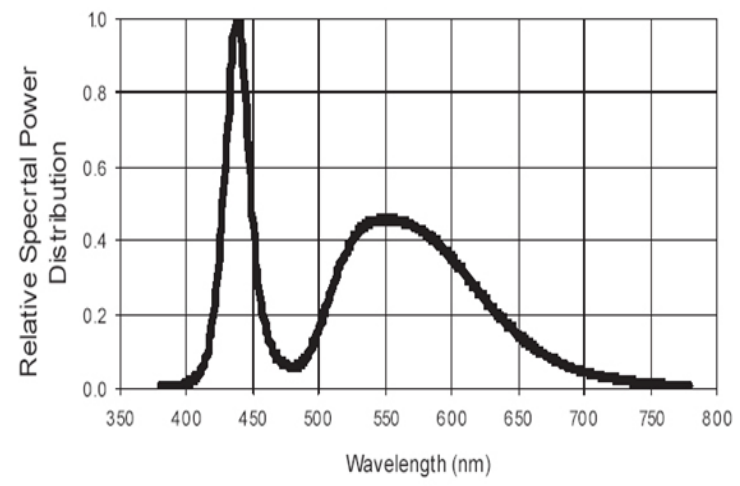

Figure 53: EL spectra of a commercial white LED based on a blue LED with yellow phosphor. 


\subsection{Summary and Conclusions of Task 1}

- Methods have been developed to grow smooth or textured GaN templates by the HVPE method. These GaN templates are grown at high growth rates with small amount of Ammonia. As a result their use in the development of LEDs should result to the reduction in their cost. Furthermore these GaN templates have relatively small concentration of dislocations.

- LEDs were fabricated and evaluated in three wavelength regions:

Emission at 350-360 nm by using GaN /AlGaN MQWs. The evaluation of these devices optically has shown that devices grown on textured GaN templates have an extraction efficiency of about $90 \%$. Furthermore, the internal quantum efficiency increase by a factor of 30 compared to those grown on smooth $\mathrm{GaN}$ templates.

$>$ Emission at 380-400 nm by using Monolayer InN /GaN MQWs. These devices have the promises to produce efficient LEDs for tricolor phosphor excitation. Also these devices are expected to be very stable during processing or operation since InN likes to be phase separated from GaN.

$>$ Emission at 400- $550 \mathrm{~nm}$ by using InGaN / GaN MQWs. These devices were grown both on smooth and textured GaN templates. The ones employing textured MQWs emit in two wavelengths (blue and green) under certain biasing conditions produce white light (Phosphorless white LEDs) 


\section{Task 2 - Theory and Simulations}

Task 2.1: Quantum Well Studies

Our earlier experiments measuring cathodoluminescence from $\mathrm{AlGaN}$ quantum wells deposited on textured (multi-faceted) GaN material have reported an order of magnitude increase in the internal quantum efficiency [1]. Furthermore narrow spot size electroluminescence showed that radiation is emitted predominantly from "quantum wedges" (QWG) formed at the locations where plane quantum wells (QW) intersect at an angle, such as shown in Fig 2.1.1(a). Note that here there is no energy barrier encircling the confinement regime, as in quantum wires of triangular cross-section deposited on Vgrooves [2-3].

Solutions of the Schrodinger equation for carriers inside V-shaped quantum wells, Fig 2.1.1(b), carried out in this work, show the existence of 1-D quantum wedge eigenmodes (QWG), confined both across and along the QW direction at the V-tips. Thus a spontaneous reduction in dimensionality occurs: the ridges among intersecting 2-D quantum wells form a 1-D quantum-wire network in the third direction. Confinement of the fundamental (lowest energy) mode occurs for QW barrier heights as low as $0.15 \mathrm{eV}$ (five times thermal energy), and for wedge angles as wide as $120^{\circ}$. The energy separation between the 1D confined fundamental QWG and the 2-D confined QW-like modes increases as the wedge gets sharper.

The effects of an electric field along the wedge axis are then included to address the polarization field inside polar materials. Titling of the wedge "floor", Fig. 2.1.1(c), due to the longitudinal E- component along the wedge "arms", causes a longitudinal version of the quantum confined Stark effect. Many lower modes now become 1-D confined, Fig 2.1.1(d). Electron and hole wavefunctions are separated at wedges located at opposing ends of a QW. It is further shown that at carrier densities $>10^{18} \mathrm{~cm}^{-3}$ electrostatic shielding by the free (passing) carriers leads to a complete screening of the longitudinal field from the QW flat segments. The emerging shielded potential is localized within nanometer-sized, exponentially decaying charged layers (plasma sheaths) around the quantum wedges [4], and leads to energy-band bending, Fig 2.1.1(e), with deeper confinement for electrons and holes. Wedges at opposing QW ends correspond to opposite polarities.

Taken together, these effects constitute an electrostatically-shielded quantum confined Stark effect. Contrasting the ordinary 2-D QCSE in QWs, where the confined energy levels decrease with increasing polarization strength, the 1-D confined QCSE energy levels increase with increasing polarization. The underlying cause is the polarization component parallel to the $\mathrm{QW}$, causing additional confinement in the second direction along the $\mathrm{QW}$, forcing a shrink of the wavefunction extent, and an uplifting of the energy levels [5]. The upshift in the energy levels obtained from numerical solutions of Schrodinger Eq. is shown in Fig. 2.1.1(f), which also shows the obtained downshift in a straight QW as a benchmark. The upshift in the energy separation between electron and hole levels, agrees with the observed blue-shifting of the emitted radiation [1]. Since the length of each QW is much larger than its width, recombination between 1-D confined electrons and holes in opposed wedges is precluded. Here emission involves transitions between 1-D QWG electrons and 2-D QW-like holes, and vice versa.

It is thus argued that the 1-D network of ridges formed among intersecting QW planes in textured materials, behaves spontaneously as a network of quantum wires along the third direction, as shown in Fig. 2.1.1(g). That is accompanied by an enhanced, 1-D density of states, and by an increase in the energy separation between electrons and holes. These predictions agree with the experimentally observed blue shifting in the spontaneous emission, and its predominant concentration at quantum wedges. The transition probability for 1-D bound - 2-D bound recombination will be addressed to determine whether it can also lead to an increased emission rate, compared to the usual recombination among 2-D bound states in "ordinary" straight QWs. 
(a)

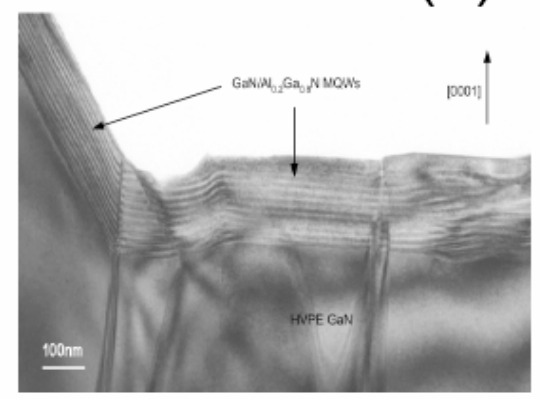

(c)
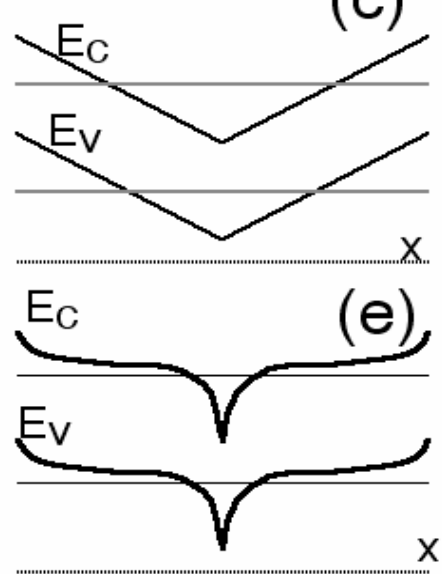

(b)

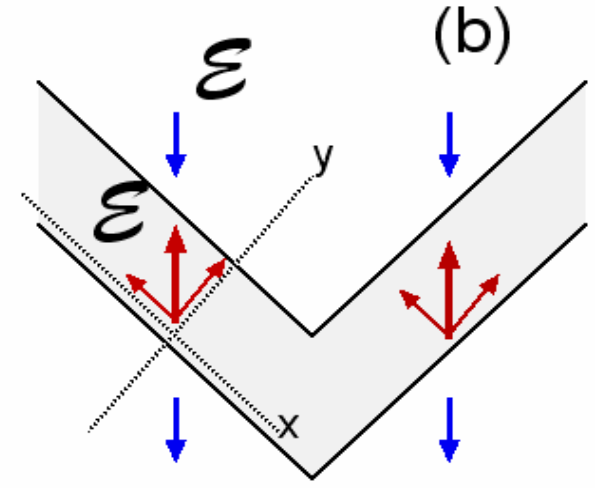

(d)

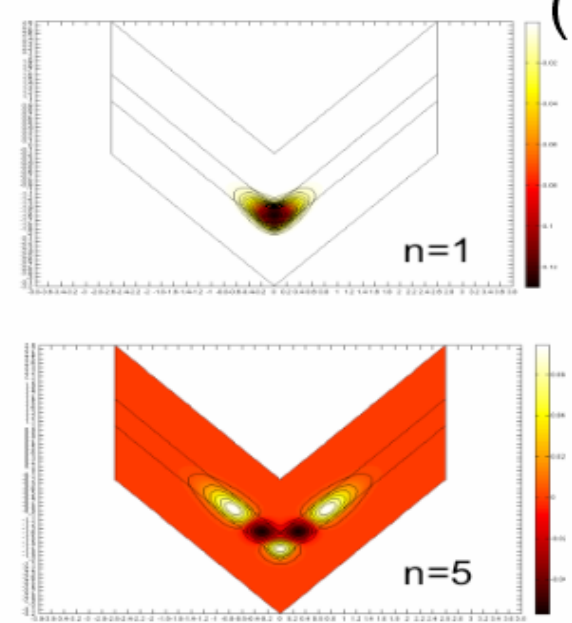

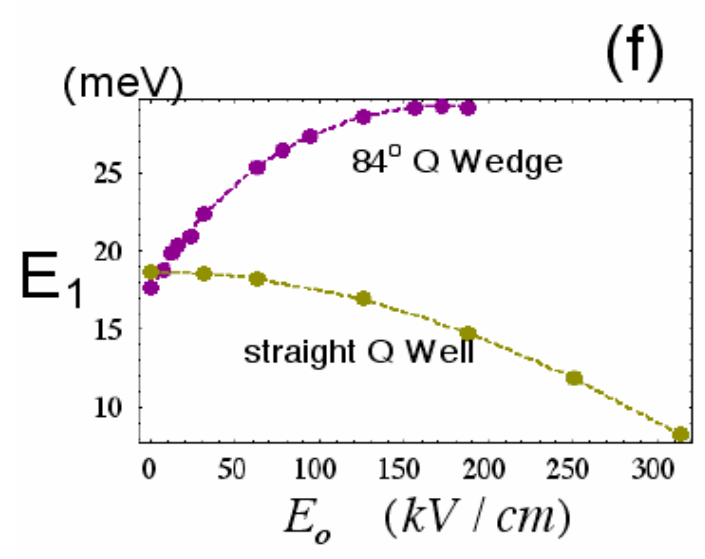

(g)

Fig 2.1.1. (a) SEM of intersecting QWs deposited on textured GaN (b) Illustration - quantum wedge geometry (c) Tilted energy bands along the wedge due to the parallel polarization field (d) Confined carrier wavefunctions in 1-D (e) Band-bending due to shielding of the polarization field (f) Fundamental energy level vs polarization strength, quantum wedge mode (upper), straight quantum well (lower) curve (g) Quantum wire formation at intersecting QWs. 


\section{Task 2.2: $\underline{\text { Emission Enhancement Studies }}$}

The parallel component to the quantum well direction $E_{x}$ causes a tilting of the energy bands as in Fig. 2.1.1(d). Thermodynamic free carrier response leads to a Poisson's equation for the self-consistent equilibrium potential along the well $\Phi(\mathrm{x})$ where the carrier charge density obeys Fermi occupation statistics including the $\Phi(\mathrm{x})$ influence. Induced electrostatic (ES) shielding excludes the polarization field from most of the QW length and causes localized band-bending at the wedges, Fig. 2.1.1(e). Typical solutions for $\Phi(\mathrm{x}), n_{e}(x)$ and $n_{h}(x)$, obtained using the 2-D carrier density of states [7], are plotted in Fig. 2.2.1(a-c) for n-doped GaN/AlGaN QWs. Although shielding limits the sheath potential $V_{s}$ well below the "driving parallel potential" $V_{x}=E_{x} L$ (which can be much larger than the transverse $V_{y}=E_{y} d$ because the QW segment length $L>>d$ ) the $V_{s}$ values remain an order of magnitude higher than the thermal energy $\kappa \mathrm{T}$. The values $\mathrm{eV}_{\mathrm{s}} / \kappa \mathrm{T}$ are plotted vs. polarization $E_{x}=E_{o}$ in Fig. 2.2.2(a) for different values of Ndopant density $N_{D}$.

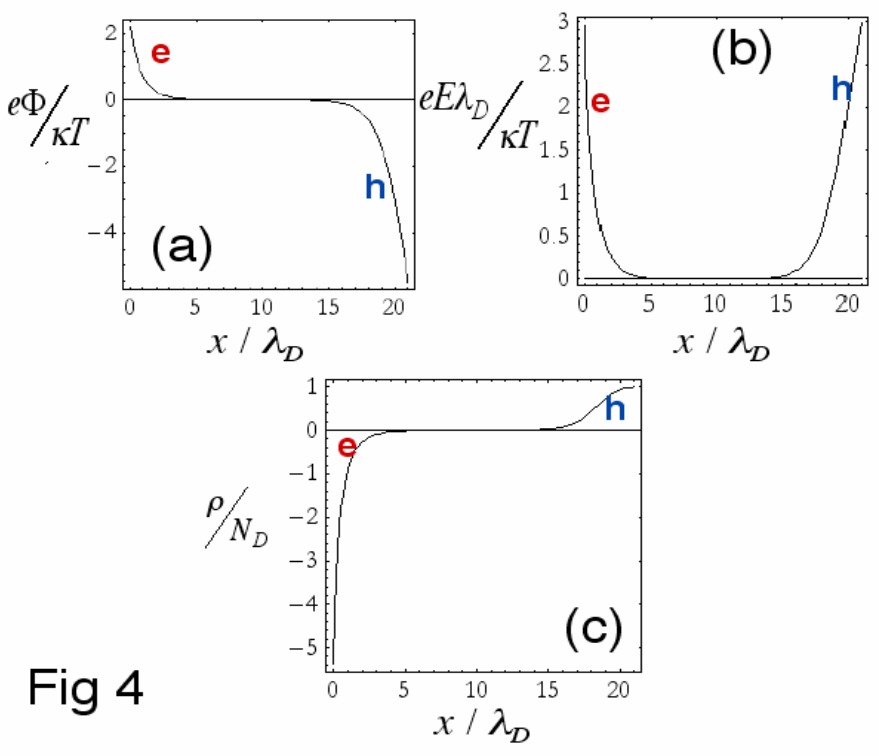

Fig 5

(a)

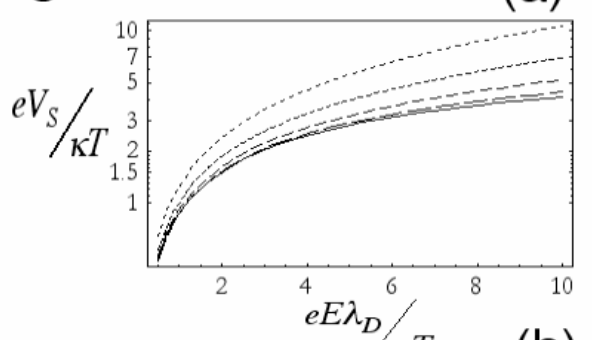

$\kappa T$

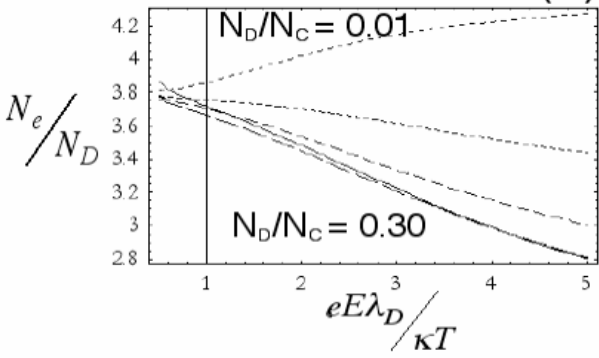

Fig. 2.2.1 Self-consistent solution for the (a) induced voltage, (b) E-field and (c) carrier density along a quantum well segment between two quantum wedges. n-doping causes asymmetry in the electron and hole voltages; total charge neutrality is preserved by a change in Fermi-level.

Fig. 2.2.2 (a) Maximum electron sheath potential, normalized to thermal, vs. polarization field strength (b) Enhancement in total number of electrons along total QW length due to band-bending, relative to zero polarization field value. Various curves denote different ratios of doping density to the

The local bending of the conduction and valence bands relative to the Fermi level results in enhanced electron and hole densities at opposite ends of a QW segment. As a result the total number of electrons and holes $N_{e, h}$ integrated over the entire QW segment length $L$ is also increased compared to a flat-band equilibrium. This follows by direct integration of the $n_{e}(x), n_{h}(x)$ profiles, such as those in Fig 4c. Typical values $N_{e} / N_{D}$ are shown in Fig. 2.2.2(b). Assuming a small, uniform over the QW induced change in occupation numbers $\delta f_{e, h}<<1$, due to either photo-excitation or current injection, the (over equilibrium) spontaneous emission rate via recombination conduction electrons and valence holes $\left.R_{s} \propto \int d^{3} k\left\{\delta f_{e}[\varepsilon(k)] f_{h}^{o}[\varepsilon(k) ; \Phi]+\delta f_{h}[\varepsilon(k)] f_{e}^{o}[\varepsilon(k) ; \Phi]\right\} \delta\left(\varepsilon_{e}(k)-\varepsilon_{h}(k)-\tilde{\sim} \bar{\omega}\right)\right]$ 
also increases. Preliminary comparisons against spontaneous emission for same excitation rate but for flat bands $f_{h, e}^{o}[\varepsilon(k) ; \Phi=0]$ indicate a factor of 4 increase for the range of typical $E_{x}$ and $N_{D}$. Thus the observed spontaneous emission enhancement can be partially attributed to increased carrier concentration at the quantum wedges.

The observed blue shifting in the spontaneous emission is explained theoretically using the trapped electron and hole energy levels. The trapped electron - trapped hole transition is extremely rare because of the large longitudinal separation in the carrier wave-functions. Most of the emission occurs from the transitions between trapped-electron and passing hole, or trapped hole and passing electron states. (Here, the characterizations "passing" and "trapped" refer to the direction along the QWG arms; all states are confined across the QWs.) Passing, approaching holes experience the electron sheath voltage as a repulsive potential; the hole "group velocity" slows down and the wavefunction amplitude increases (probability conservation) as the hole approaches the classical "turning" point. The overlap between the confined electron wavefunction and the tunneling (evanescent) portion of the transmitted hole wavefunction leads to significant transition amplitudes.

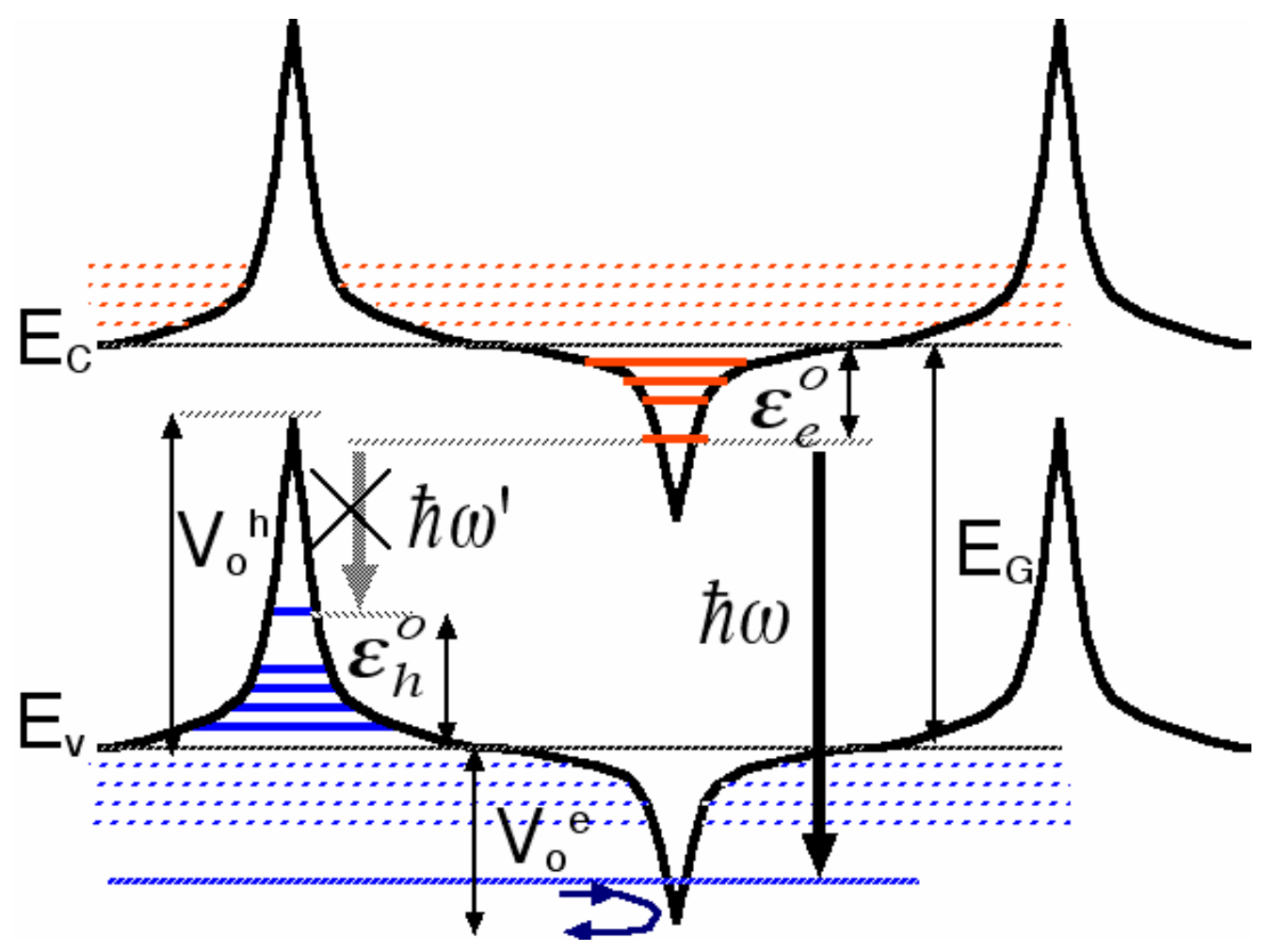

Fig. 2.2.3 Transition energies between wedge-confined electron and holes $\left.\approx \tilde{\omega}^{\prime}\right)^{\prime}$ (negligible probability), as well as between confined electrons and passing holes $\tilde{\tilde{w}} \boldsymbol{\mu}$ (dominant observed). Solid and dashed lines represent, respectively, confined and passing energy levels.

It turns out from quantum mechanical calculations that peak transition probability occurs for passing hole energy nearly equal to the peak sheath voltage, whereby the classical "turning point" of the hole lies on the wedge axis, at the center of the confined electron wavefunction. The shift in the emission energy then equals the trapped carrier energy relative to the bottom of the electrostatic potential well, as depictued schematically in Fig. 2.2.3. For trapping potential of about $20 \mathrm{kT}$ the expected blue shift is of the order of $10 \mathrm{kT}$ or $0.26 \mathrm{eV}$. This corresponds to wavelength shift of about $\Delta \lambda=\lambda(\delta \varepsilon / \varepsilon)=363 \mathrm{~nm}(0.26 / 3.34)=$ 
$28 \mathrm{~nm}$, close to the observed $33 \mathrm{~nm}$. In the future the work focus will shift on explaining the enhanced transition probability from transitions involving confined carrier states.

Task 2.3: Light Extraction Angle Studies.

Our earlier photoluminescence experiments involving textured surface $\mathrm{GaN}$ grown on $\mathrm{GaN}$ templatesdemonstrated significant enhancement in the light transmission efficiency [1] from GaN into air, with a parallel significant reduction in the reflectivity of the GaN interface under external illumination. Specifically, the observed reflectivity from a textured $\mathrm{GaN}$ surface was limited to only $1 \%$, compared to the $20 \%$ value obtained for a flat $\mathrm{GaN} /$ air interface and $\mathrm{GaN}$ refraction index of 2.6. In addition, a near $100 \%$ extraction efficiency is inferred from the observed 50 times enhancement in the PL efficiency relative to smooth surface GaN under identical illumination. Since total internal reflection above the critical angle $\theta_{c}=1 / \mathrm{n}=22^{\circ}$ limits extraction to $3.8 \%$ of the entire solid angle (i.e. the extraction cone), a near $100 \backslash \%$ extraction accounts for a 25 -fold increase in extraction.

It is already well known that periodically or randomly structured (corrugated) interfaces significantly enhance light extraction efficiency [6-7] well beyond the total reflection cone limiting extraction from flat surfaces. Earlier theoretical treatment of light extraction has employed the ray tracing approach [6]. If the incidence angle on a surface facet exceeds the critical a ray is totally reflected; it keeps bouncing with ever-changing incidence on different facet directions until it is partially transmitted when the incidence angle turns subcritical.

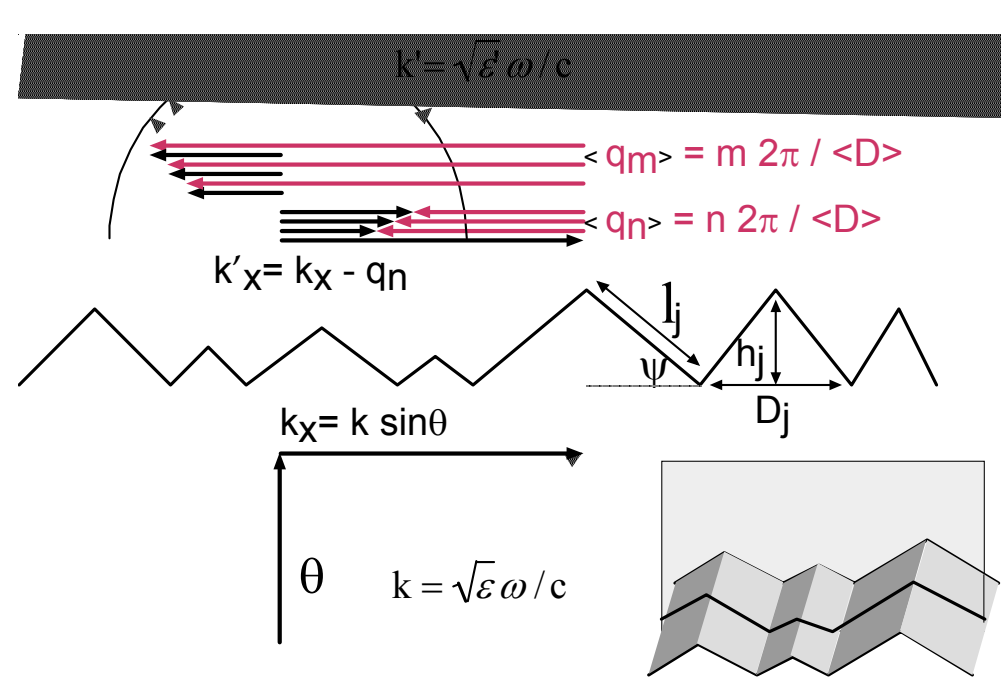

Fig. 2.3.1 Scattering components on a rough triangular surface
Ray tracing however is not valid when the typical surface feature sizes are comparable to the wavelength, as depicted in Fig. 2.3.1. This situation arises in microtextured GaN surfaces with typical feature sizes of $1 \mu \mathrm{m}$ comparable to optical wavelengths. Here transmission is better described by scattering [8] due to the nonconservation of the parallel wavenumber $k_{\|}$. A single incident wavelength is scattered into many directions characterized by $k_{\|}{ }^{\prime}=k_{\|} \pm q_{n}$ where $q_{n}=n(2 \pi / D)$ is an inverse lattice vector for surface feature size $D$ (for non-periodic surfaces $q_{n}$ spans a semicontinuum.)

As a consequence, the scattered wavenumber falls within the maximum

allowed wavenumber in the optically thin medium, $k_{\|}{ }^{\prime} \leq \sqrt{\varepsilon^{\prime}}(\omega / c)$, even when the incident parallel exceeds this maximum $k_{\|}=\sqrt{\varepsilon}(\omega / c) \sin \theta>\sqrt{\varepsilon^{\prime}}(\omega / c)$, and so it allows partial transmission above the critical angle $\sin \theta_{c}=\sqrt{\varepsilon^{\prime}} / \sqrt{\varepsilon}$.

The essence of the transmission for supercritical incidence is illustrated in Fig. 2.3.1. The interface nonuniformity along the $\mathrm{x}$-direction violates $k_{\|} \equiv k_{x}=k_{\|}{ }^{\prime}$ conservation during reflection / transmission, and imposes scattered wavenumbers $k_{\|}{ }^{\prime}=k_{\|} \pm q_{n}$ where $q_{n}=n(2 \pi / D)$ and D is the typical feature size "period". For a flat surface and $\mathrm{q}=0$ no transmission is allowed when 
$k_{\|}{ }^{\prime}=k_{\|}=\sqrt{\varepsilon}(\omega / c) \sin \theta>\sqrt{\varepsilon^{\prime}}(\omega / c)$, i.e. for incidence with $\sin \theta>\sin \theta_{c} \equiv \sqrt{\varepsilon^{\prime} / \varepsilon}$. The nonconservation $k_{\|} \neq k_{\|}$'for textured surface allows an entire range of transmitted "harmonics" $q_{n}$ for supercritical incidence, such that

(2.2.1) $|\sqrt{\varepsilon}(\omega / c) \sin \theta-q|<\sqrt{\varepsilon^{\prime}}(\omega / c)$

For wavelength-size features $D \approx \lambda$ and $q \approx k$ or for high enough $\mathrm{n}$, the above eqwuation is satisfied for $\sin \theta>\sin \theta_{c}$ up to nearly grazing incidence. For a quasi-random interface the harmonics $q_{n}$ are replaced by a statistical ensemble of given $<\mathrm{q}>$.

The task of this work is (a) to go beyond ray tracing approaches, applicable for large feature size interfaces, and introduce a supercritical scattering approach that is applicable at short feature scales. (b) to inplement the new approach in a numerical simulation algorithm for computation of radiation extraction from rough microstructured interfaces. As a result, a new effective current model leading to a quantitative extraction formula was developed, which, while less accurate than the non-local integral approach employed in [9] it is never-the-less much easier to implement computationally and transparent in bringing out the underlying physical insight.

Certain differences between micro-facet scattering and ray tracing are to be noted, before going into specifics. First, there is always partial transmission $t(\theta)>0$ from any given single incidence, regardless of the incidence angle $\theta$ - no successive bouncing is required to achieve subcritical incidence. Second, simultaneous reflection into many Floquet wavenumbers $q_{n}$ causes a fast diffusion of the internally reflected light; a plane wave incident on a rough surface at any angle is quickly converted into a collection of partial waves uniformly distributed over the entire range of incidence. Thus, for practical evaluations, one may take the averaged transmission coefficient $\bar{T}=\langle T(\theta)>$ over the full range of incident angles $0<\theta<\pi$ as the transmission coefficient for a single incidence. The total transmitted power over many successive bounces between opposed interfaces is then given by summing the terms of a geometrical progress $\mathrm{T}=\bar{T}+(1-\alpha) \bar{R} \bar{T}+(1-\alpha)^{2} \bar{R}^{2} \bar{T}+\ldots \ldots=\bar{T} /[1-(1-\alpha) \bar{R}]$, where $\alpha$ is the material absorptivity between successive incidences and $\bar{R}=1-\bar{T}$. Without absorption, $\alpha=0$, a $100 \%$ transmission would be possible from a structured surface. By contrast, for perfect, parallel opposed surfaces the incidence angle would never change; a wave incident above critical angle would remain indefinitely trapped inside the slab.

The method of induced interface source currents [8] during this effort in order to treat scattering from corrugated surfaces. It is based on the observation that the continuity of the parallel electric field $E_{\|}=E_{\|}$'at an interface causes a discontinuity in the parallel displacement $D_{\|}=\varepsilon E_{\|}$vector due to the difference in dielectric constants $\varepsilon \neq \varepsilon$. The surface discontinuity in $\delta\left[D_{\|}\right]$is treated as a source current term

\section{(2.2.2) $\left[\nabla \times D_{\|}\right]=I_{\|} \propto \hat{p}(\varepsilon / \varepsilon, \theta, k)$}

in the wave equation for the magnetic field. To replace the finite "extinction length" layer in dielectrics with a localized $\delta$-function effective current, the discontinuity magnitude is scaled by a factor $\hat{p}\left(\varepsilon^{\prime} / \varepsilon, \theta, k\right)$. The value of $\hat{p}$ is then determined so that that the source term reproduces the exact refracted and reflected waves for a flat surface. For scattered wave-vectors $\mathbf{k}$ - q the corresponding surface current strength I(k-q) follows from the geometrical interface details, directly related to the FT of the surface geometry. Thus the irregular surface effects are embodied in the current distribution over the appropriate surface geometry. All the statistical boundary attributes are absorbed in the current source, generating the scattered fields via the uniform (half)-space propagator (i.e. Green's functions) in each dielectric area. This is much easier to handle analytically than performing direct field matching over irregular boundaries. Although other perturbative approaches treating random interface scattering exist [10 - 12], the present 
approach provides a direct connection between the spectral statistical properties of the 2-D interface geometry and the transmitted fields. In addition, the derived surface interference factor gives a closedformula unified description of the transition from coherent Bragg scattering, for nearly-periodic interfaces $<\delta D^{2}>/ D<<1$ to quasi-uniform light diffusion at high surface randomness $<\delta D^{2}>/ D \approx 1$.

The method has been first applied earlier to 1-D interfaces with randomly distributed square teeth of varying height and aspect ratio [8]. Here we consequently expand this approach for the 2-D textured $\mathrm{GaN}$ interfaces used in our optically pumped LED experiments. For simplicity the nano-structured surface, composed of triangular pyramids, is replaced by triangular prisms, and the corrugated interface is formed by a quasiperiodic repetition of geometrically similar, triangular prisms, with infinitely long parallel vertices. The reflection physics is determined by the plane of incidence, defined by the incident wavenumber and the local surface normal. The intersection of the corrugated interface with any incidence plane yields a 2-D "cross-sectional" surface, formed by the repetition of geometrically similar triangular units (same angle $\psi$ ) of varying base size $D_{j}$ and height $h_{j}=\left(D_{j} / 2\right) \tan \psi$, as shown schematically in Figure 2.3.1(a). The triangular sizes follow a size distribution of average $\left\langle D_{j}\right\rangle=D$ and given standard deviation $\left\langle\delta D^{2}\right\rangle=\Delta^{2}$. The general extraction properties are studied first on the cross-section perpendicular to the prism vertices. The extraction coefficient for 3-D solid angle incidence follows by integration of the 2-D triangular interface extraction formula over all incidence planes.

Under multispectral reflection / transmission, a fraction $E_{q}$ of the incoming plane wave amplitude $E_{0}$ is transmitted and reflected, respectively, into the Floquet components $k_{\|}{ }^{\prime}(q)=k_{\|}-q$ and $k_{\|}{ }^{\prime \prime}(q)=k_{\|}-q$ of amplitudes $E_{q}{ }^{\prime}$ and $E_{q}{ }^{\prime \prime}$. The relative transmission / reflection coefficients among Floquet components of scattering vector $\mathrm{q}, t(q)=E_{q}^{\prime} / E_{q}, \quad r(q)=E_{q}^{\prime \prime} / E_{q}$, are obtained by matching of the surface-parallel EM components along any flat segment of the corrugated surface (a choice of a different surface segment with the same orientation simply corresponds to a phase shift for all $\left.E_{q}, E_{q}{ }^{\prime}, E_{q}{ }^{\prime \prime}\right)$ yielding

$$
\left(\begin{array}{l}
r(q) \\
t(q)
\end{array}\right)_{T E}=\left(\begin{array}{c}
\frac{\cos \theta-\sqrt{\varepsilon^{\prime} / \varepsilon} \cos \theta^{\prime}(q)}{\cos \theta^{\prime \prime}(q)+\sqrt{\varepsilon^{\prime} / \varepsilon} \cos \theta^{\prime}(q)} \\
\frac{\cos \theta^{\prime \prime}(q)+\cos \theta^{\prime}(q)}{\cos \theta^{\prime \prime}(q)+\sqrt{\varepsilon^{\prime} / \varepsilon} \cos \theta^{\prime}(q)}
\end{array}\right), \quad\left(\begin{array}{l}
r(q) \\
t(q)
\end{array}\right)_{T M}=\left(\begin{array}{l}
\frac{\sqrt{\varepsilon^{\prime} / \varepsilon} \cos \theta-\cos \theta^{\prime}(q)}{\sqrt{\varepsilon^{\prime} / \varepsilon} \cos \theta^{\prime \prime}(q)+\cos \theta^{\prime}(q)} \\
\frac{\cos \theta^{\prime \prime}(q)+\cos \theta}{\sqrt{\varepsilon^{\prime} / \varepsilon} \cos \theta^{\prime \prime}(q)+\cos \theta^{\prime}(q)}
\end{array}\right)
$$

where the corresponding q-scattered reflection and refraction angles are given by

$$
\sin \theta^{\prime}(q)=\frac{k \sin \theta-q}{\sqrt{\varepsilon^{\prime} / \varepsilon} k}, \quad \sin \theta^{\prime}(q)=\frac{k \sin \theta-q}{k}
$$

In the limit $\mathrm{q}=0$ one recovers the usual reflection / refraction from infinite flat surface.

As derived, Eq. () is an exact relation between $E_{q}, E_{q}{ }^{\prime}$ and $E_{q}{ }^{\prime \prime}$. Its usefulness is however limited by the fact the incident amplitude fraction $E_{q} / E_{0}$ channeled into the q-component is not revealed by (). We have seen however that $E_{q}$ is associated to emission from an effective surface current component $I(q)$ - finding $E_{q}$ thus amounts to computing $I(q)$. The crux of our approximation is that $I(q)$ follows from the geometrical FT of the surface current along the corrugated surface. It turns out that

$$
\begin{gathered}
I^{\prime}(q){ }_{T E}^{T M}=-i k E_{o}\left(\begin{array}{l}
2 \varepsilon^{\prime} t_{T M}(q) \cos \theta^{\prime}(q) \hat{\mathbf{y}} \\
2 \varepsilon^{\prime} t_{T E}(q) \cos ^{2} \theta^{\prime}(q) \hat{\mathbf{x}}
\end{array}\right) F(q), \\
I^{\prime \prime}(q)_{T E}^{T M}=-i k E_{o}\left(\begin{array}{l}
2 \varepsilon r_{T M}(q) \cos \theta^{\prime}(q) \hat{\mathbf{y}} \\
2 \varepsilon r_{T E}(q) \cos ^{2} \theta^{\prime}(q) \hat{\mathbf{x}}
\end{array}\right) F(q)
\end{gathered}
$$

where I' and I" respectively apply for the transmitted (refracted) and reflected waves, and we used the qscattered reflection and transmission coefficients to express $E_{q}^{\prime}=t(q) E_{q}, E_{q}^{\prime \prime}=r(q) E_{q}$. The geometry enters via the Fourier transform of the surface 


\section{(2.2.5) $\quad F(q)=f(q) S(q)$}

where $f(q)$ is the FT of the individual surface feature (a triangular or square tooth) and $\mathrm{S}(\mathrm{q})$ the screen interference factor from all teeth. The Born approximation is implied here, since we are using the unperturbed incoming plane wave for the computation of the induced surface current.

Substituting ()-() as the source currents in Maxwell's Eq. yields a simple formula for the amplitudes of the transmitted / reflected E'(k-q), E' (k-q)

$$
\begin{gathered}
\text { (2.2.6) } \quad E^{\prime}(k-q)=t(q) F(q) E_{o}(k) \\
E^{\prime \prime}(k-q)=r(q) F(q) E_{o}(k)
\end{gathered}
$$

where $E_{o}$ is the incoming amplitude. In effect, an incident plane wave of amplitude Eo is equivalent to incident spectral Floquet components $\mathrm{E}(\mathrm{k}-\mathrm{q})=\mathrm{F}(\mathrm{q}) \mathrm{Eo}(\mathrm{k})$ of reflectivity $\mathrm{r}(\mathrm{q})$ and transmissivity $\mathrm{t}(\mathrm{q})$. In the infinite, flat surface limit $F(q) \rightarrow \delta(q)$ one recovers the exact flat surface formulae. Forming the Poynting flux $\mathbf{E} \mathbf{x} \mathbf{B}$, and integrating over the entire allowed scattered q-spectrum, the desired reflected and transmitted power is given by

$$
\begin{aligned}
& T(\theta)=\sqrt{\frac{\varepsilon^{\prime}}{\varepsilon}} \frac{1}{\cos \theta} \int_{q m n}^{q m x} d q_{n}\left|t\left(q_{n}\right)\right|^{2} f^{2}\left(q_{n}\right)\left\langle\mathrm{S}^{2}\left(q_{n}\right)\right\rangle \cos \theta^{\prime}\left(q_{n}\right) \\
& R(\theta)=\frac{1}{\cos \theta} \int_{q m n}^{q m x} d q_{n}\left|r\left(q_{n}\right)\right|^{2} f^{2}\left(q_{n}\right)\left\langle\mathrm{S}^{2}\left(q_{n}\right)\right\rangle \cos \theta^{\prime}\left(q_{n}\right)
\end{aligned}
$$

The effect of the rms deviations in feature size is built into the screen factor $\left\langle S^{2}\left(q_{n}\right)\right\rangle$, which goes from the well-known Bragg interference pattern, for periodic texturing, to nearly isotropic angular diffusion for high randomness (roughness.)

The integral formulae have been implemented into numerical algorithms for the cases of square and triangular teeth. Numerical results have investigated single pass extraction from a (quasi-periodic) triangular prism interface as function of the index contrast, triangular angle, wavelength-to-feature size ratio and degree of randomness (roughness).

Numerical results for the total transmitted power from triangular interfaces of Fig. 2.3.1 are shown in Fig. 2.3.2(a-b), plotting the total transmission coefficient into all scattering angles vs. plane wave incidence at angle \$ltheta\$. A 50\% mixture of TE and TM polarization is assumed for the incident wave. Fig. 2.3.2(a) shows $\mathrm{T}(\theta)$ for fixed $<\mathrm{D}>/ \lambda=2 / 3$ and fixed rms deviation in the triangular size $\Delta / \angle \mathrm{D}>=0.250$, for various facet angles $\psi$ as marked. Supercritical extraction occurs outside the internal reflection cone $|\theta-\psi|>\theta_{c}=22^{\circ}$,becoming stronger with increasing facet angle $\psi$. Figure 2.3.2b shows $\mathrm{T}(\theta)$ for fixed $\psi=\pi / 4$ and $<\mathrm{D}>/ \lambda=2 / 3$ for various surfaces "roughness" $\Delta /<\mathrm{D}>$ as marked. Predictably, the extracted fraction increases with increasing roughness. A general observation that stands out is that the presence of

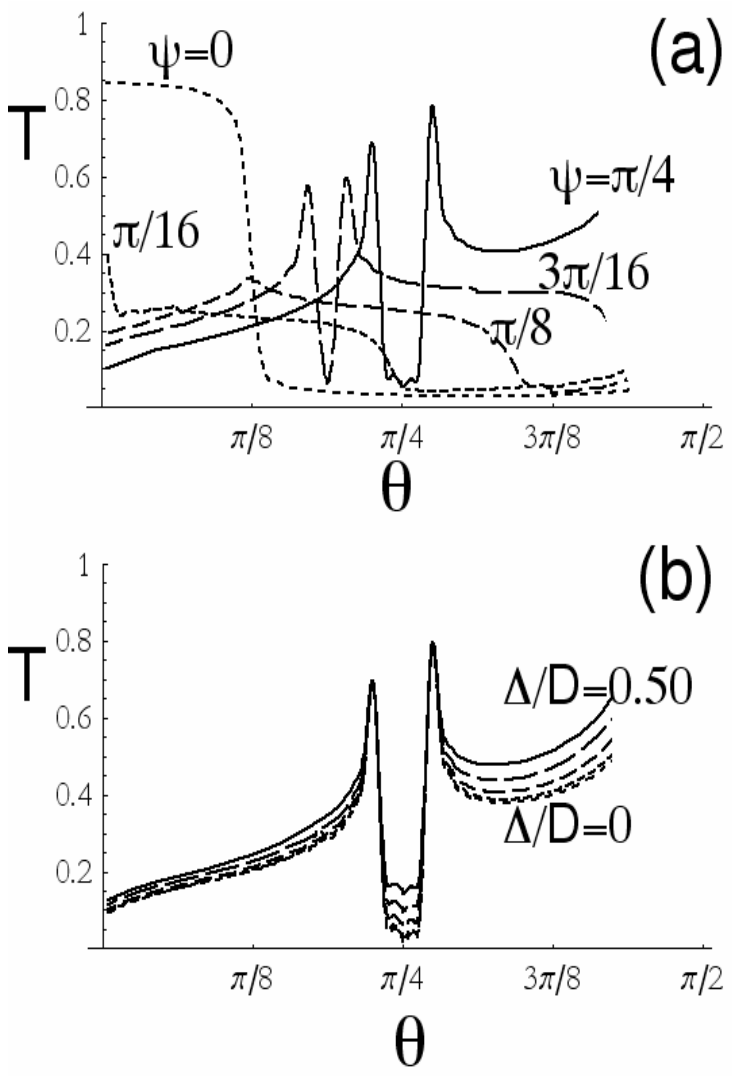

Fig. 2.3.2 Single-pass transmission coefficient $\mathrm{T}$ vs incidence angle for a $50 \%$ mixture of $\mathrm{TE}$ and TM polarization for the incident plane wave: (a) various facet angles $\psi$ at $\pi / 8$ steps, for fixed rms triangular size deviation $\Delta /<\mathrm{D}>$ $=0.250$ and fixed $<D>/ \lambda=2 / 3$ (b) various rms deviations in the triangular size $\Delta /<\mathrm{D}>$ (light dashed to solid line in steps of 0.125$)$ for fixed $\psi=\pi / 4$ and $<\mathrm{D}>/ \lambda=2 / 3$. 
nano-structuring actually reduces extraction at sub-critical incidence angles (compared to flat surfaces), in agreement with earlier theory [9]. This trend gets stronger and stronger with smaller feature size D (longer $\lambda$ ). Yet, the supercritical angle extraction more than makes up for the subcritical reduction, resulting in a net increase in the overall extraction. Thus the integral of the transmission coefficient $T(\theta)$ over the $\pi$-incidence angle increases the total per-pass light extraction up to a factor of 4 relative to the flat surface result, as will be seen below.

In summary EM component matching along microscopic sub-wavelength surface segments was used for obtaining macroscopic (global) $r(q), t(q)$. The local continuity of the parallel $\mathrm{E}$ and $\mathrm{H}$, expressed by $($ ), is tantamount to total EM energy and flux conservation everywhere across the interface. In addition, relations () can be re-derived by using energy and EM momentum conservation during individual "photon" scattering, where $r(q), t(q)$ now express the individual photon probability of scattering into the q-channel.

It is reasonable to use the averaged transmission coefficient over all incidence angles as the single-pass extraction coefficient $\bar{T}$,

$$
\text { (2.2.8) } \bar{T}=\frac{1}{2 \pi} \int_{-\pi / 2}^{\pi / 2} d \theta T(\theta)
$$

.Energy conservation implies an averaged reflection coefficient $\bar{R}=1-\bar{T}$. We now recall that the above formula applies in two dimensions, where incident waves at all polar angles $\theta$ are located on the same azimuthal plane of incidence. To extend the result to incidence over the entire solid angle we have to include incidence at different azimuthal angles $\phi$. Incident wave vectors lying on incidence planes at azimuthal angles $\phi$ experience scattering from 2-D triangular interfaces of effective base-length and angle respectively

$\hat{D}_{\phi}=D / \cos \phi, \quad \hat{\psi}_{\phi}=\tan ^{-1}(\tan \psi \cos \phi), \quad$ where $\quad \mathrm{D}$ and $\psi$ correspond to incidence plane perpendicular to the prism vertices $\phi=0$. The $3-\mathrm{D}$ extraction percentage follows by an additional $\phi$ integration of the earlier derived 2-D formula with the appropriate effective $\hat{D}_{\phi}$ and $\hat{\psi}_{\phi}$,

$$
\bar{T}=\frac{1}{4 \pi} 2 \int_{0}^{2 \pi} d \phi \int_{-\pi / 2}^{\pi / 2} d \theta \sin \theta T\left(\theta, 0 ; \hat{\psi}_{\phi}, \hat{D}_{\phi}\right)
$$

Here the 2 factor in front of the integral accounts for extraction from two parallel interfaces (or one interface and a perfectly reflecting opposed interface).

Figure 2.3.3(a) shows the extraction efficiency averaged over the incidence angle $\bar{T}$ as a function of the average facet size ratio $<D>/ \lambda$, for various triangular facet inclinations $\psi$ with given $\Delta / D=0.250$ in Fig. 11a. The extraction increases with facet angle $\psi$; a higher than a factor of two increase in extraction occurs relative to a flat surface (incidentally, because the transmission coefficient $T(\theta)$ is less than unity the extraction from a flat surface is smaller than the extraction cone; for $50 \% \mathrm{TE}$ and TM mixture the exact $\bar{T}=0.059<0.076$. Figure 2.3 .3 (b) plots $\bar{T}$ vs. $<\mathrm{D}>/ \lambda$ for various degrees of surface roughness $\Delta<\mathrm{D}>$ at given $\psi=\pi / 4$. An increase in extraction with increasing roughness occurs mainly at 
wavelengths comparable to the feature size, with optimum $<\mathrm{D}>/ \lambda \sim 1 / 2$. For short wavelengths the extraction is practically independent of rms roughness.

Fig. 2.3.4 shows the single-pass transmission coefficient, for uniform angular incidence, versus relative facet size $<\mathrm{D}>/ \lambda$ for various index contrasts among materials, as marked. Facet angle $\psi=\pi / 4$, and rms roughness $\Delta /<\mathrm{D}\rangle=0.355$. Single-pass extraction increases with decreasing index contrast. In all cases extraction is enhanced by a factor two or more above the corresponding flat surface extraction value (respectively 0.0291, 0.0588, 0.124 and 0.253, for GaAs-air, GaN-air, $\mathrm{GaAs}-\mathrm{Al}_{2} \mathrm{O}_{3}$ and $\mathrm{GaN}-\mathrm{Al}_{2} \mathrm{O}_{3}$.) Highest index contrasts (air) receive the maximum enhancement. The optimum feature size for maximum extraction becomes longer $<\mathrm{D}>/ \lambda \sim 2$, at lower index contrast. This is consistent with Eq. ( $\operatorname{ref}\{\mathrm{QRNG}\}$ ), since a smaller scattering vector $\mathrm{q} \sim 2 \pi / \mathrm{D}$ is required under smaller index contrast.

Now, multiple bounces between top and bottom surfaces further increase extraction well above the single-pass value. Let the between-bounce percentage losses be given by a fraction $\alpha$. The total transmitted power over many successive bounces between opposed interfaces is then given by summing the terms of the geometrical progress $\mathrm{T}=\bar{T}+(1-\alpha) \bar{R} \bar{T}+(1-\alpha)^{2} \bar{R}^{2} \bar{T}+\ldots \ldots$ yielding

$$
\text { (2.2.10) } \quad \mathrm{T}=\frac{\bar{T}}{1-(1-\alpha)(1-\bar{T})}
$$

Because of the repeated bouncing and transmission it only takes a single-pass extraction of $\bar{T}=0.08$, or just twice the single flat surface extraction, and a per-pass absorption $\alpha=0.05$, in order to yield $\mathrm{T}=$ 0.76 , i.e. about 20 times the single flat surface extraction, or 10 times the extraction from two opposed flat surfaces. In fact single pass extraction values near $25 \%(0.25)$ have been evaluated in Fig. 2.3.3, and thus multiple bounce extraction approaches unity, consistent with the observed enhancement in PL efficiency.

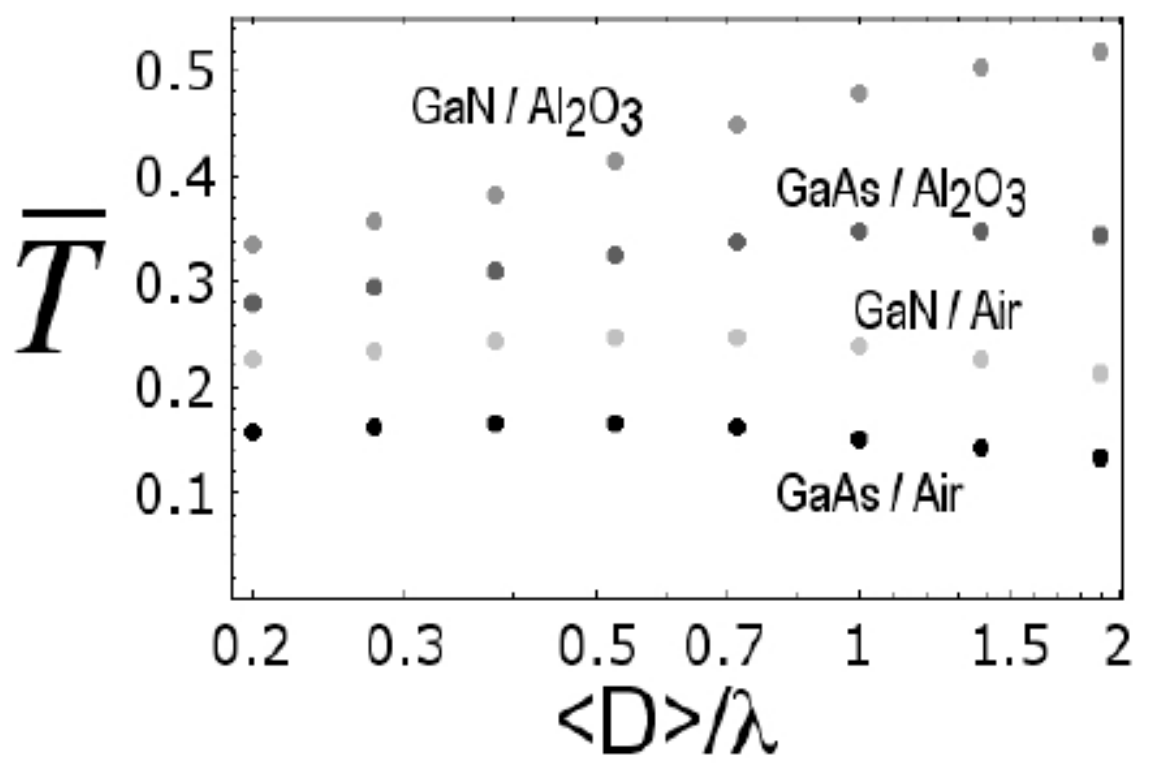

Fig. 2.3.4 Single-pass transmission coefficient, for uniform angular incidence, versus relative facet size $\langle\mathrm{D}>/ \lambda$ for various index contrasts among materials, as marked. Facet angle $\psi=\pi / 4$, and rms roughness $\Delta /<\mathrm{D}>=0.355$. 


\section{$\underline{\text { List of publications }}$}

1. Jasper S. Cabalu, Christos Thomidis, Theodore D. Moustakas and Spilios Riyopoulos, "Enhanced Light Extraction and Spontaneous Emission from Textured GaN Templates Formed During Growth by the HVPE Method", Proceedings of the Electrochemical Society, Vol. 2004-06, p.351 (2004).

2. J. C. Cabalu, C. Thomidis, I. Friel and T.D. Moustakas, (INVITED), “ Nitride LEDs based on Flat and “wrinkled" Quantum Wells” Quantum Sensing and Nanophotonic DevicesII, Edited by Manijeh Razeghi and Gail Brown, Proc. of SPIE, Vol. 5732, 185(2005)

3. S. Riyopoulos, J. Cabalu and T. D. Moustakas, "Enhanced light extraction through nano-textured GaN interfaces via supercritical angle scattering” Optoelectronic Devices: Physics, Fabrication and Applications, Edited by Joachim Piprek, Proc. of SPIE, Vol. 6013, G-1 (2005).

4. J. C. Cabalu, C. Thomidis, I. Friel and T.D. Moustakas and S. Riyopoulos, "Enhanced internal quantum efficiency and light extraction efficiency from textured GaN/AIGaN quantum wells grown by molecular beam epitaxy” J. Appl. Phys 99, 064904 (2006).

*Also selected to appear in the Virtual Journal of Nanoscale Science \& Technology April 3, 2006, Editor David Awschalom

5. J. S. Cabalu, A. D. Williams, Tai-Chou P.Chen, R. France and T. D. Moustakas, "Visible Light Emitting Diodes Grown by Plasma-assisted MBE on Hydride vapor-phase epitaxy GaN templates and the development of dichromic (Phosphorless) white LEDs.” Mater. Res. Soc. Symp. Proc. Vol. $\underline{\mathbf{8 9 2}}, 245$ (2006).

6. Ryan France, Tao Xu, Papo Chen, R. Chandrasekaran and T. D. Moustakas, "Vanadium-based Ohmic contacts to n-AIGaN in the entire alloy composition”, Appl. Phys. Lett. $\underline{90}, 062115$ (2007)

7. R. Chandrasekaran, A.S. Ozcan, D. Deniz, K.F. Ludwig, and T. D. Moustakas, “Growth of non-polar (11-20) and semi-polar (11-26) AlN and GaN films on the R-plane sapphire” Physica Status Solidi (c) $\underline{4}$, No.5, 1689-1693 (2007).

8. S. Riyopoulos, T. D. Moustakas and J. S. Cabalu, "Plasma nanosheath formation with carrier accumulation and enhanced localized spontaneous emission at "quantum wedges" in textured GaN”. Physics of Plasmas $\underline{\mathbf{1 4}}$, 053501(2007)

9. S. Riyopoulos, T. D. Moustakas and J. S. Cabalu, "Enhanced transmission through quasirandom nanostructured dielectric interface via supercritical angle scattering”, J. Appl. Phys. 102, 043111 (2007).

* Also selected to appear in the Virtual Journal of Nanoscale Science \& Technology (Sept. 10, 2007, Editor David Awschalom)

10. Josh Abell and T. D. Moustakas, "The role of dislocations as non-radiative recombination centers in InGaN MQWs” Appl. Phys. Lett. 92, 091901 (2008)

11. Lin Zhou, David J. Smith, R. Chandrasekaran, T. D. Moustakas, "Structural characterization of non-polar (1120) and semi-polar (1126) GaN films grown on $r$-plane sapphire”, J. of Crystal Growth (accepted) 
12. E. Dimakis, A. Yu Nikiforov, C. Thomidis, L. Zhou, D. J. Smith. J. Abell, C. -K. Kao and T. D. Moustakas, "Growth and properties of near-UV light emitting diodes based on InN / GaN quantum wells” Physica Status Solidi (a) (Accepted)

13. C. Thomidis, A. Yu. Nikiforov, Tao Xu, and Theodore D. Moustakas, "InGaN-based LEDs grown by plasma-assisted MBE on (0001) sapphire with GaN QDs in the nucleation layer” Physica Status Solidi (a) (Accepted).

\section{References:}

\section{Task 1:}

[1] S. S. Liu and D.A. Stevenson J. Electrochemical Soc. 125, 1161 (1978)

[2] V. S. Ban J. Electrochemical Soc. 119, 761 (1972)

[3] M. Iligems, J. Cryst. Growth 13/14, 360 (1972)

[4] W. Seifert et al J. Cryst. Growth 52, 257 (1981)

[5] M. R. Lorentz and B. B. Binkowski, J. Electroc. Soc. 109, 24 (1962)

[6] Naniwae et al J. Cryst. Growth 99, 381 (1990)

[7] T. D. Moustakas, R. J. Molnar, G. Menon and C.R. Eddy Jr., "A Comparative Study of GaN Films Grown on Different Faces of Sapphire by ECR-assisted MBE," Mat. Res. Soc. Symp. Proc., 242, T. D. Moustakas, J. I. Pankove and Y. Hamakawa, eds., (1992), p. 427.

[8] T. D. Moustakas, T. Lei and R. J. Molnar, "Growth of GaN by ECR-assisted MBE," Physica B, 185, $36(1993)$.

[9] Tavernier et al. J. Am. Ceram. Soc. 85, 49 (2002)

[10] Detchprohm et a, Appl. Phys. Lett. 61, 2688 (1992)

[11] C. R. Eddy, T. D. Moustakas and J. Scanlon, "Growth of Gallium Nitride Thin Films by Electron Cyclotron Resonance Microwave Plasma-assisted Molecular Beam Epitaxy," J. Appl. Phys., 73, 448 (1993).

[12] A.Bhattacharyya, W.Li, J.Cabalu, T.D. Moustakas, David J. Smith and R.L. Hervig, "Efficient P-type Doping of GaN films by Plasma- assisted Molecular Beam Epitaxy" Appl. Phys. Lett. 85, 4956 (2004)

[13] A.Bhattacharyya, I. Friel, S. Iyer, T.C.Chen, W. Li, J.Cabalu, Y.Fedunin, K.F.Ludwig J T.D. Moustakas, H. P. Maruska, D.W. Hill, J.J.Gallagher, M. M. Chou, B.Chai "Comparative study of GaN/AlGaN MQWs grown homoepitaxially on (1-100) and (0001) GaN", Journal of Crystal Growth, Vol. 251, 487 (2003). 
[14] B. E. A. Saleh and M. C. Teich Fundamental of Photonics (Wiley Interscience, New York, 1991), p. 60 .

[15] J. Cabalu, Ph. D. Thesis (Boston University 2006)

[16] T. Xu Ph. D. Thesis (Boston University 2007)

[17] 5. S. F. Chichibu et al, Nature Materials $\underline{5}, 810$ (2006).

[18] A. Hangleiter, F. Hitzel, C. Netzel, D. Fuhrmann, U. Rossow, G. Ade, and P. Hinze, Phys. Rev. Lett. 95127402 (2005).

[19] T. Hino, S. Tomiya, T. Miyajima, K. Yanashima, S. Hashimoto, and M. Ikeda, Appl. Phys. Lett. $\underline{76}, 3421(2000)$

\section{Task 2:}

[1] J. S. Cabalu, C. Thomides, T. D. Moustakas, S. Riyopoulos, Lin Zhou and D. J. Smith "Enhanced internal quantum efficiency and light extraction efficiency from textured GaN/AlGaN quantum wells grown by molecular beam epitaxy", J. Applied Phys. 99, 064904 (2006).

[2] A. Gustafsson, F. Reinhardt, G. Biasiol, and E. Kapon, "Low-pressure organometallic chemical vapor deposition of quantum wires on V-grooved substrates", APL 67, 3673 (1995).

[3] T. Schrimpf, P. Bonsch, D. Wullner, H.-H. Wehmann, A. Schlachetzki, F. Bertram, T. Riemann, and J. Christen "InGaAs quantum wires and wells on V-grooved InP substrates", JAP 86, 5207 (1999).

[4] S. Riyopoulos, T. D. Moustakas and J. S. Cabalu," "Plasma nanosheath formation with carrier accumulation and enhanced localized spontaneous emission at "quantum wedges" in textured GaN", Phys. of Plasmas 14, 053501 (2007).

[5] S. Riyopoulos and T. D. Moustakas, "1-D carrier localization with effective quantum wire behavior inside quantum wedges in textured GaN materials", in preparation (2007).

[6] I. Schnitzer, E. Yablonovitch, C. Caneau, T. J. Gmitter and A. Scherer "30\% external quantum efficiency from surface textured, thin-film light-emitting diodes", Appl.Phys. Lett. 63, 2174-76 (1993).

[7] R. Windish, C. Rooman, B. Dutta, A. Knobloch, G. Borghs, G. H. Dohler and P. Heremans "Lightextraction mechanisms in high efficiency, surface textured LEDs", IEEE JSTQE 8, 248-255 (2002).

[8] S. Riyopoulos, "Supercritical angle transmission through quasi-random sub-wavelength feature interfaces: Integral approach via effective surface currents”, JOSA A 22, 2859-2871 (2005).

[9] M. Nieto-Vesperinas "Scattering and diffraction in physical optics" (Wiley, New York 1991).

[10] A.G. Voronovich, "Scattering from rough surfaces" (Springer-Verlag, Berlin 1994).

[11] J. A. Ogilvy, "Theory of wave scattering from random rough surfaces" (Hilger, London, 1991). 
[12] A. Sentenac and J.-J. Greffet, "Mean-field theory of light scattering by one dimensional rough surfaces", JOSA A 15 528-532 (1998).

[13] See for example J. D. Jackson in "Classical Electrodynamics" (Wiley, New York, NY 2nd edition, 1975) pp 278-282.

[14] See for example R. Kubo, M. Todda and W. Hasitsume in "Statistical Physics II" (Springer-Verlag, Berlin, 1985) pp 22-55. 